Classificação Semi-Supervisionada Baseada em Desacordo por Similaridade 



\title{
Classificação Semi-Supervisionada Baseada em Desacordo por Similaridade
}

\author{
Víctor Antonio Laguna Gutiérrez
}

Orientador: Prof Dr Alneu de Andrade Lopes

Dissertação apresentada ao Instituto de Ciências Matemáticas e de Computação - ICMC-USP, como parte dos requisitos para obtenção do título de Mestre em Ciências de Computação e Matemática Computacional.

USP - São Carlos

Fevereiro/2010 



\section{Dedicatória}

Aos meus amados pais, Víctor e Blanca, e ao meu irmão Luis. 



\section{Agradecimentos}

Gostaria de agradecer primeiramente a minha família, motivo pelo qual os primeiros parágrafos estão em espanhol.

A mis amados padres, Víctor y Blanca, por ser mi fuerza, mi alma, mi razón de ser y mis mejores amigos. A pesar de la distancia siempre estuvieron presentes conmigo cuando más lo necesitaba, mostrando todo el amor que solo los padres saben dar. También agradezco a mi hermano Lucho, que siempre estuvo presto a ayudarme y a compartir experiencias conmigo. Yo sé que estas pocas líneas no alcanzan para mostrar todo mi agradecimiento por ustedes.

Agradezco también a toda mi familia por parte de padre y madre. Entre ellos quiero enfatizar mis agradecimientos a mis tías Flora, María, Yolanda, Ercilia, Betty, a mis tíos Antonio, Pancho, Edgard y a mis padrinos Carmen y Félix. Adicionalmente agradezco de todo corazón a mi amorito Mel (que ya considero parte de mi familia), que supo apoyarme, ayudarme y estar a mi lado para conseguir este logro que ahora es conjunto.

Ao professor Alneu de Andrade Lopes, pelos conselhos, orientações, e trabalho conjunto durante todo este tempo. Além de me orientar, teve que lidar com os problemas do meu "imperfeito" português. Nada que uma garrafa de um bom Pisco peruano não saiba arrumar!

Aos professores Solange, Gustavo e Carolina, que além de ter sido muito importantes para minha formação acadêmica e pessoal, eu os considero modelos a seguir.

Aos meus colegas de laboratório, companheiros de estudo, parceiros de jogo e principalmente amigos de coração: Márcio, Bruno, Everton, Igor, Robson, Leonardo, André, Andrés, Fernanda, Rafael Giusti, Rafael Rossi, Ricardinho, Edson, Fabiano, Nils, Tatiane, Jean, Cintra, Miguelito, Allinson e Diego, OBRIGADO POR TUDO!!! Agradeço também aos meus amigos de outros laboratórios e outros cursos e, especialmente, ao Seu Arly.

Aos meus amigos que considero como irmãos Alfredo Roa (Shakira), José Rabanal (Tom), Eder Quispe (DJ), Fernando Mejía (Narizón), Didier Vega (Yiyier), Zico Herrera (Beibeahh!) e Arturo Blumen. Amigos como vocês são difíceis de conseguir!

À Capes (Coordenação de Aperfeiçoamento de Pessoal de Nível Superior), pelo apoio financeiro.

Finalmente, agradeço a todos que me ajudaram direta ou indiretamente. 



\section{Resumo}

aprendizado semi-supervisionado é um paradigma do aprendizado de máquina no qual a hipótese é induzida aproveitando tanto os dados rotulados quantos os dados não rotulados. Este paradigma é particularmente útil quando a quantidade de exemplos rotulados é muito pequena e a rotulação manual dos exemplos é uma tarefa muito custosa. Nesse contexto, foi proposto o algoritmo Cotraining, que é um algoritmo muito utilizado no cenário semi-supervisionado, especialmente quando existe mais de uma visão dos dados. Esta característica do algoritmo Cotraining faz com que a sua aplicabilidade seja restrita a domínios multi-visão, o que diminui muito o potencial do algoritmo para resolver problemas reais. Nesta dissertação, é proposto o algoritmo Co2KNN, que é uma versão mono-visão do algoritmo Cotraining na qual, ao invés de combinar duas visões dos dados, combina duas estratégias diferentes de induzir classificadores utilizando a mesma visão dos dados. Tais estratégias são chamados de k-vizinhos mais próximos (KNN) Local e Global. No KNN Global, a vizinhança utilizada para predizer o rótulo de um exemplo não rotulado é conformada por aqueles exemplos que contém o novo exemplo entre os seus $k$ vizinhos mais próximos. Entretanto, o KNN Local considera a estratégia tradicional do KNN para recuperar a vizinhança de um novo exemplo. A teoria do Aprendizado Semi-supervisionado Baseado em Desacordo foi utilizada para definir a base teórica do algoritmo Co2KNN, pois argumenta que para o sucesso do algoritmo Cotraining, é suficiente que os classificadores mantenham um grau de desacordo que permita o processo de aprendizado conjunto. Para avaliar o desempenho do Co2KNN, foram executados diversos experimentos que sugerem que o algoritmo Co2KNN tem melhor performance que diferentes algoritmos do estado da arte, especificamente, em domínios mono-visão. Adicionalmente, foi proposto um algoritmo otimizado para diminuir a complexidade computacional do KNN Global, permitindo o uso do Co2KNN em problemas reais de classificação. 



\section{Abstract}

emi-supervised learning is a machine learning paradigm in which the induced hypothesis is improved by taking advantage of unlabeled data. Semi-supervised learning is particularly useful when labeled data is scarce and difficult to obtain. In this context, the Cotraining algorithm was proposed. Cotraining is a widely used semisupervised approach that assumes the availability of two independent views of the data. In most real world scenarios, the multi-view assumption is highly restrictive, impairing its usability for classification purposes. In this work, we propose the Co2KNN algorithm, which is a one-view Cotraining approach that combines two different k-Nearest Neighbors (KNN) strategies referred to as global and local k-Nearest Neighbors. In the global KNN, the nearest neighbors used to classify a new instance are given by the set of training examples which contains this instance within its k-nearest neighbors. In the local $K N N$, on the other hand, the neighborhood considered to classify a new instance is the set of training examples computed by the traditional KNN approach. The Co2KNN algorithm is based on the theoretical background given by the Semi-supervised Learning by Disagreement, which claims that the success of the combination of two classifiers in the Cotraining framework is due to the disagreement between the classifiers. We carried out experiments showing that Co2KNN improves significatively the classification accuracy specially when just one view of training data is available. Moreover, we present an optimized algorithm to cope with time complexity of computing the global KNN, allowing Co2KNN to tackle real classification problems. 



\section{Sumário}

Resumo .............................. ix

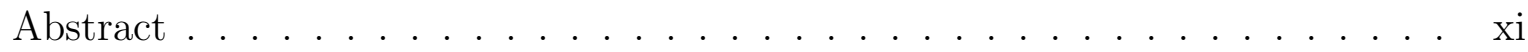

Sumário . . . . . . . . . . . . . . . . . . . i

Lista de Figuras . . . . . . . . . . . . . . . . . . . . iv

Lista de Tabelas . . . . . . . . . . . . . . . . . . . . . v

Lista de Algoritmos . . . . . . . . . . . . . . . . . . . . vii

1 Introdução 1

1.1 Contexto e Motivação . . . . . . . . . . . . . . . . . . . . 1

1.2 Objetivos e Metodologia . . . . . . . . . . . . . . . . . 3

1.3 Organização do Trabalho . . . . . . . . . . . . . . . . . . . . 4

2 Aprendizado Semi-Supervisionado 5

2.1 Considerações Iniciais . . . . . . . . . . . . . . . . . . . . 5

2.2 Aprendizado Supervisionado e Não Supervisionado . . . . . . . . . . . . . 6

2.3 Aprendizado Semi-Supervisionado . . . . . . . . . . . . . . . . . . . . 8

2.3.1 Pressupostos do Aprendizado Semi-Supervisionado . . . . . . . . . 9

2.3.2 Aprendizado Indutivo vs. Transdutivo . . . . . . . . . . . . . . 11

2.3.3 Técnicas do Aprendizado Semi-Supervisionado . . . . . . . . . . . . 12

2.4 O Cotraining . . . . . . . . . . . . . . . . . . 18

2.4.1 Pressupostos do Cotraining . . . . . . . . . . . . . . . 19

2.4.2 O Algoritmo Cotraining . . . . . . . . . . . . . 19

2.4.3 O Cotraining e o Aprendizado Baseado em Desacordo . . . . . . . . 21

2.5 Trabalhos Relacionados . . . . . . . . . . . . . . . . . . . 23

3 Classificadores Baseados em Instâncias $\quad 27$

3.1 Considerações Iniciais . . . . . . . . . . . . . . . . . . . 27

3.2 Medidas de Similaridade . . . . . . . . . . . . . . . . . . 28

3.2 .1 Dados Numéricos . . . . . . . . . . . . . . . . . . . 28

3.2 .2 Dados Categóricos . . . . . . . . . . . . . . . . . . . . . . . . 29

3.2 .3 Outros Tipos de dados . . . . . . . . . . . . . . . 31 
3.3 Algoritmo K Vizinhos Mais Próximos . . . . . . . . . . . . . . . . . . . 32

3.3.1 Definição do Algoritmo . . . . . . . . . . . . . . . . . . 32

3.3.2 Variações do Algoritmo . . . . . . . . . . . . . . . . . . 34

3.3 .3 Grafos baseados em Similaridade . . . . . . . . . . . . . 36

3.4 Trabalhos Relacionados . . . . . . . . . . . . . . . . . . . . . . . . . . 38

4 Classificação Semi-Supervisionada Baseada em Desacordo Usando KNN Global e Local $\quad 43$

4.1 Considerações Iniciais . . . . . . . . . . . . . . . . . . . . . . . . 43

4.2 Estratégias de Recuperação de Vizinhos . . . . . . . . . . . . . . . . . . . 44

4.2 .1 KNN Local . . . . . . . . . . . . . . . . . . . . . . . . . 44

4.2 .2 KNN Global . . . . . . . . . . . . . . . . . . 46

4.2 .3 Análise de Desacordo entre as Estratégias . . . . . . . . . . . . . 47

4.3 Combinando o KNN Local e Global com Cotraining . . . . . . . . . . . . . 50

4.3.1 Metodologia . . . . . . . . . . . . . . . . 50

4.3 .2 O Algoritmo Co2KNN . . . . . . . . . . . . 51

4.4 Exemplo Ilustrativo . . . . . . . . . . . . . . . . . 54

5 Avaliação Experimental $\quad 61$

5.1 Considerações Iniciais . . . . . . . . . . . . . . . . . . . . . 61

5.2 Abordagem para Avaliação do Algoritmo Co2KNN . . . . . . . . . . . 61

5.3 Configurações . . . . . . . . . . . . . . . . . . . 63

5.3 .1 Conjunto de Dados . . . . . . . . . . . . . . . . . 63

5.3.2 Parâmetros de Entrada . . . . . . . . . . . . . . . . . 64

5.3.3 Classificadores Co2KNN Utilizados . . . . . . . . . . . . . . 65

5.4 Experimentos . . . . . . . . . . . . . . . . . . 67

5.4.1 Comparação com os Algoritmos Tradicionais . . . . . . . . . . . . . 67

5.4 .2 Comparação com as Estratégias Isoladas . . . . . . . . . . . . . . 69

5.4.3 Comparação entre o KNN Local e o KNN Global . . . . . . . . . . 71

5.4 .4 Comparação com o SKNN . . . . . . . . . . . . . . . . . 73

5.4.5 Comparação entre Co2KNN e Co2KNN-FA . . . . . . . . . . 77

6 Conclusões $\quad 79$

6.1 Principais Contribuições . . . . . . . . . . . . . . . . . . . 81

6.2 Trabalhos Futuros . . . . . . . . . . . . . . . . . . . . . . 82

$\begin{array}{ll}\text { Referências } & 83\end{array}$

A Figuras com o desempenho dos classificadores em relação a K $\quad 95$ 


\section{Lista de Figuras}

2.1 Pressuposto de suavidade. . . . . . . . . . . . . . . . . . . 10

2.2 Pressuposto de formação de grupos. . . . . . . . . . . . . . . . . . . 10

2.3 Predição da distribuição das classes com o algoritmo EM . . . . . . . . . . 13

2.4 Hiperplanos gerados pela SVM para separar as classes. Figura adaptada de Zhu e Goldberg (2009). . . . . . . . . . . . . . . . . . . 14

2.5 Grafo formado pelos 3 vizinhos mais próximos de cada vértice. . . . . . . . 15

2.6 Grafo bipartido. Imagem modificada de Blum e Mitchell (1998). . . . . . . 22

3.1 Pela a distância euclidiana, o exemplo mais próximo do novo exemplo é da classe " $\times$ ". Pela distância de Mahalanobis é "•". . . . . . . . . . . . . . . . 30

3.2 Diferentes conjuntos de vizinhos para valores de $k=1,3$ e 5 . . . . . . . 33

3.3 Limites de decisão para diferentes valores de $k \ldots \ldots$. . . . . . . . . . 33

3.4 Exemplo de um modelo de similaridade baseado em grafos, sendo 3.4a a distribuição dos dados e 3.4b o modelo alcançado pelas redes KNN. Imagem modificada de Hein e Luxburg (2007) . . . . . . . . . . . . . . . . . . 37

3.5 Exemplo de formação da rede KNN simétrica para $k=3$ onde 3.5a apresenta a distribuição de dados inicial, 3.5b e 3.5c o processo de formação do grafo e $3.5 \mathrm{~d}$ a distribuição real das classes . . . . . . . . . . . . . . 38

3.6 Vértice mais informativo dentre dos $k$ vizinhos mais próximos do novo exemplo . . . . . . . . . . . . . . . . . . . 3

3.7 Diferentes conjuntos de vizinhos encontrados pelas estratégias KNN e SKNN para $k=1$. Imagem modificada de Nock et al. (2000) . . . . . . . . . . . . 40

3.8 Exemplo de obtenção da vizinhança na rede k-associados com a visão testerede e rede-teste para uma rede com $k=3$. Figura modificada de Motta e Lopes $(2009) \quad \ldots \ldots \ldots \ldots$. . . . . . . . . . . . . . . . 41

4.1 Diferentes exemplos novos a serem rotulados o KNN Local. Os exemplos em 4.1a e 4.1b são rotulados com confiança alta, pois a grande maioria de vizinhos pertencem a mesma classe. Entretanto, os exemplos mostrados em 4.1c e 4.1d são rotulados com uma confiança menor. . . . . . . . . . . . 
4.2 Conjunto de vizinhos para diferentes exemplos segundo a estratégia KNN

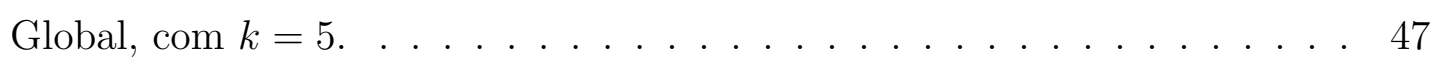

4.3 Re-distribuição de arestas em uma rede KNN com $k=3$. O quadrado representa o novo vértice $\vec{x}_{\text {new }} \ldots \ldots \ldots \ldots$. . . . . . . . . . . 48

4.4 Impacto do novo exemplo na distribuição de arestas de classes esparsas e compactas. . . . . . . . . . . . . . . . . . . . . . . . . 49 49

4.5 Metodologia proposta do presente trabalho. . . . . . . . . . . . . . . 51

4.6 Distribuição inicial dos dados, com dados não rotulados em branco e duas classes coloridas. . . . . . . . . . . . . . . . . 55

4.7 Estado do conjunto de dados após a primeira iteração do algoritmo Co2KNN. 56

4.8 Distribuição de arestas para o exemplo 1. . . . . . . . . . . . . . . . 56

4.9 Distribuição de arestas para o exemplo 2. . . . . . . . . . . . . . . . . . 57

4.10 Distribuição de arestas para o exemplo $3 \ldots$. . . . . . . . . . . . 57

4.11 Distribuição dos dados após a iteração 4. . . . . . . . . . . . . . . . . 58

4.12 Novo exemplo a ser rotulado. . . . . . . . . . . . . . . . . . . . . 59

5.1 Metodologia de avaliação do presente trabalho. . . . . . . . . . . . . . . . 62

5.2 Conjunto de vizinhos recuperado com cada estratégia. O conjunto obtido pelo KNN Global está representado pelas arestas incidentes ao novo exemplo e as arestas de saída representam o conjunto obtido pelo KNN Local. 66

5.3 Comparação entre o número fixo de vizinhos recuperados pelo KNN Local e o número variável recuperado pelo KNN Global para $k \in[1,3,5,15]$. . . 73

5.4 Comparação de AUC entre o desempenho em relação ao crescimento de $K$ em 4 bases. . . . . . . . . . . . . . . . . . . . . . . 74

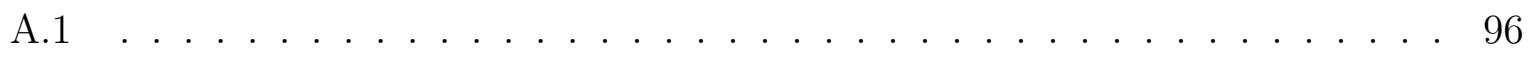

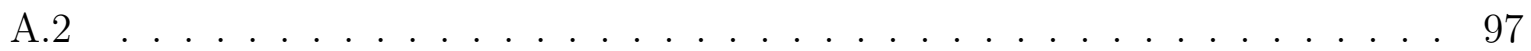

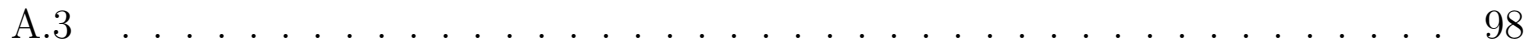




\section{Lista de Tabelas}

5.1 Detalhe dos conjuntos de dados. . . . . . . . . . . . . . . . 64

5.2 Parâmetros dos conjuntos de dados no algoritmo Co2KNN. . . . . . . . . . 65

5.3 Comparação de AUC de diferentes configurações do Co2KNN com os algoritmos tradicionais. . . . . . . . . . . . . . . . . . . 68

$5.4 \mathrm{Co} 2 \mathrm{KNN}$ vs Algoritmos tradicionais com teste estatístico. . . . . . . . . . . 69

5.5 Comparação de AUC do Co2KNN com as estratégias separadas para $k=1$. 70

5.6 Comparação de AUC do Co2KNN com as estratégias separadas para $k=3$. 70

5.7 Comparação de AUC do Co2KNN com as estratégias separadas para $k=5.71$

5.8 Comparação de AUC do Co2KNN com as estratégias separadas para $k=15$. 71

5.9 Co2KNN vs Selftraining com teste estatístico . . . . . . . . . . . . . . 72

5.10 KNN Local vs KNN Global com teste estatístico. . . . . . . . . . . . . . . 72

5.11 Comparação de AUC do Co2KNN com o SKNN para valor de $k=1$. . . 75

5.12 Comparação de AUC do Co2KNN com o SKNN para valor de $k=3$. . . . 75

5.13 Comparação de AUC do Co2KNN com o SKNN para valor de $k=5$. . . 76

5.14 Comparação de AUC do Co2KNN com o SKNN para valor de $k=15$. . . 76

$5.15 \mathrm{Co} 2 \mathrm{KNN}$ vs SKNN. . . . . . . . . . . . . . . . . . . . 77

5.16 Co2KNN vs Co2KNN-FA com teste estatístico. . . . . . . . . . . . 77 



\section{Lista de Algoritmos}

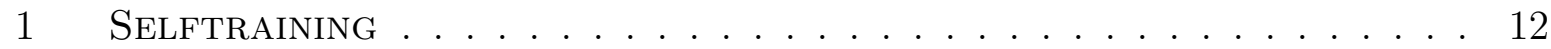

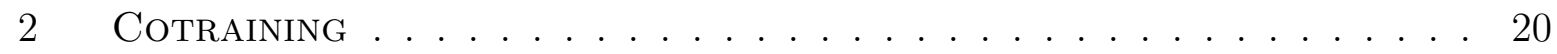

3 K-Vizinhos Mais Próximos . . . . . . . . . . . . . . . 34

4 Recuperar Vizinhos Mais Próximos KNN Local . . . . . . . . . . . 45

5 Recuperar Vizinhos Mais Próximos KNN Global . . . . . . . . . 48

6 Proposta Inicial . . . . . . . . . . . . . . . . . . 52

7 TreinAR KNN GLOBAL . . . . . . . . . . . . . . . 53

8 Recuperar Vizinhos Mais Próximos KNN Global Final . . . . . . 53

9 Algoritmo Co2KNN . . . . . . . . . . . . . . . 54 



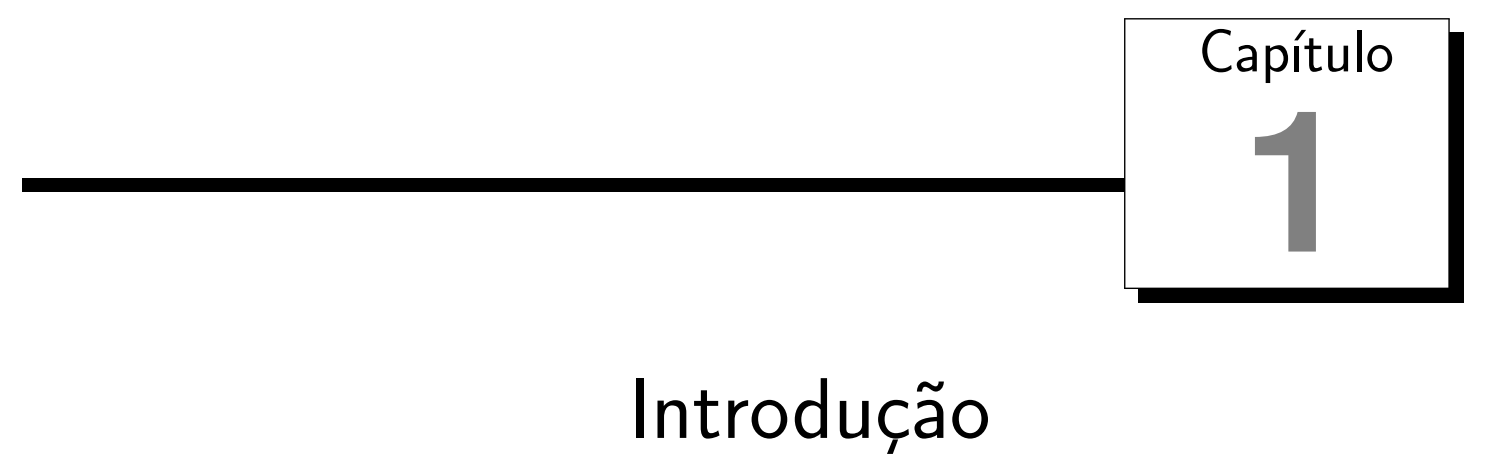

\subsection{Contexto e Motivação}

O desenvolvimento humano está vivenciando uma nova fase, na qual a economia mundial baseia-se principalmente na manipulação de informação ao invés da produção industrial. A quantidade de informação gerada pela humanidade cresce de forma acelerada, o que pode ser comprovado em pesquisas como as de Gantz et al. (2008), que estimaram um crescimento da quantidade de informação gerada no universo digital de cerca de $60 \%$ por ano. Nessa mesma pesquisa, estimou-se que para o ano 2011 o volume de informação gerado será de aproximadamente 1800 hexabytes, quase 10 vezes a quantidade de informação gerada no ano 2006. Atualmente, a crescente capacidade de armazenamento e de integração dos computadores, associadas ao advento e popularização da World Wide Web, são consideradas as principais causas da grande geração de informação pois promovem a proliferação de imensas e diversas coleções de dados em meios eletrônicos (Lyman et al., 2003).

Devido à vasta quantidade de informações disponíveis, torna-se cada vez mais difícil a seleção, exploração e análise de informações e padrões relevantes em um determinado domínio. Nesse contexto, aumenta a necessidade de desenvolvimento de técnicas automáticas para a extração de conhecimento de grandes conjuntos de dados. Diversas áreas têm devotado um grande esforço neste problema, destacando-se a área de Inteligência Artificial com o desenvolvimento diferentes técnicas de identificação de padrões conhecidas como Mineração de Dados (MD) (Witten e Frank, 2005).

Uma das tarefas da MD é a classificação, a qual consiste na generalização de experiencias passadas para predizer algum valor discreto (conhecido como classe) em novos exemplos (Hastie et al., 2003). Entretanto, a maioria da informação existente nas coleções de dados não foi criadas para sua utilização em tarefas de classificação. Para esta tarefa 
é comum a necessidade de exemplos previamente rotulados por especialistas e de uma etapa de pré-processamento dos dados para permitir uma representação inteligível aos algoritmos de classificação.

Diversas técnicas de classificação de dados têm sido empregadas usando algoritmos de Aprendizado de Máquina (AM), em especial de aprendizado supervisionado. As técnicas de aprendizado supervisionado pressupõem a disponibilidade de exemplos rotulados para a etapa de treino dos classificadores. Entre os principais algoritmos de classificação supervisionada estão as árvores de decisão (Quinlan, 1986), aprendizado baseado em regras (Furnkranz, 1999), as redes neuronais (Bishop, 1995), o aprendizado bayesiano (Berger, 1985), aprendizado baseados em instâncias (Aha et al., 1991), entre outros.

O algoritmo K-Vizinhos Mais Próximos (KNN) (Cover e Hart, 1967), é um algoritmo tradicional e muito estudado no paradigma de aprendizado baseado em instâncias pelo fato de ser considerado um algoritmo eficaz e simples (Mitchell e Blum, 1997). A idéia básica do KNN é que um exemplo novo é rotulado com base em seus $k$ vizinhos mais próximos segundo alguma medida de distância. Apesar de ser um algoritmo tradicional, ainda existem várias aplicações do KNN na pesquisa em diversos sistemas inteligentes e a investigação de técnicas relacionadas a ele continuam, encontrando-se diversas pesquisas recentes (Zhang et al., 2006; Song et al., 2007; Bodo e Minier, 2008; Weinberger e Saul, 2009; Fayed e Atiya, 2009). Entretanto, por ser um algoritmo supervisionado, o KNN necessita de um número elevado de exemplos rotulados para o treinamento dos classificadores, sendo que, na maior parte dos domínios, a obtenção de exemplos rotulados manualmente por especialistas é uma tarefa muito custosa, e em alguns casos até impraticável.

Frente a grande dificuldade de obter dados rotulados, algoritmos usando técnicas semi-supervisionadas têm sido desenvolvidos. As técnicas semi-supervisionadas também fazem uso de dados rotulados para a etapa de treinamento dos classificadores, porém, a quantidade necessária é muito menor se comparado com técnicas supervisionadas, por aproveitarem informações presentes nos exemplos não rotulados. Entre os algoritmos semisupervisionados mais relevantes pode-se citar a versão semi-supervisionada do algoritmo Expectation Maximization (Mccallum e Mitchell, 2000), a versão semi-supervisionada do algoritmo SVM (Bennett e Demiriz, 1998), a propagação de rótulos (Zhu e Ghahramani, 2002), e a estratégia tradicional de incrementar iterativamente o conjunto de exemplos rotulados com os dados rotulados com a maior confiança, conhecido como Selftraining (Nigam e Ghani, 2000).

A escassez de exemplos rotulados é um fator de grande impacto no desempenho final dos classificadores no aprendizado semi-supervisionado. Visando suprir a falta de dados rotulados, o aprendizado semi-supervisionado multi-visão foi proposto. A idéia geral do aprendizado semi-supervisionado multi-visão é utilizar várias "visões" dos dados para melhorar a hipótese dos classificadores gerados (Muslea et al., 2002). Por exemplo, para a classificação de textos de artigos científicos poderia utilizar-se os termos e suas freqüências 
para gerar uma tabela atributo-valor, formando uma primeira visão. Para tal, o especialista do domínio teria que ler e rotular uma quantidade de artigos inicial, sendo esta uma tarefa muito custosa. Com o objetivo de aumentar a informação disponível sem aumentar a quantidade de artigos inicialmente rotulados, o especialista poderia aproveitar as informações presentes nas citações para gerar uma segunda visão. Dessa forma, cada exemplo da base de textos teria duas fontes de informações que os descrevem, sendo que o especialista teria que rotular manualmente a mesma quantidade de dados.

O algoritmo Cotraining (Blum e Mitchell, 1998) foi um dos primeiros e mais efetivos algoritmos de aprendizado semi-supervisionado multi-visão a prover um framework completo para o uso de exemplos não rotulados. O Cotraining trabalha com duas visões independentes dos dados. A independência nas representações pode ter uma natureza subjetiva, requerendo de uma análise cuidadosa para verificar esse pressuposto. Ainda que exista essa restrição, alguns trabalhos têm obtido bons resultados usando representações que não são totalmente independentes (Nigam e Ghani, 2000; Goldman e Zhou, 2000; Zhou e Li, 2007).

Um dos maiores problemas na classificação semi-supervisionada multi-visão, no entanto, é a dificuldade de obter mais de uma representação dos dados que modele informações diferentes. Muitas vezes é possível formar diversas descrições dos dados, porém, aquelas descrições são dependentes do domínio. Por exemplo, as citações podem ser encontradas em textos científicos, mas não em notícias de jornais. Nesse contexto, a usabilidade do algoritmo Cotraining é restrita a domínios multi-visão, motivo pelo qual a sua aplicabilidade é reduzida em problemas reais. Frente a esse problema, foi proposto o Aprendizado Baseado em Desacordo (Zhou e Li, 2009), o mesmo que oferece fundamentos teóricos que mostram que o sucesso do algoritmo Cotraining não radica na diversidade de visões dos dados, mas na diversidade de informações considerada pelos classificadores.

\subsection{Objetivos e Metodologia}

O objetivo deste trabalho é propor uma estratégia geral para a geração de dois classificadores a partir de uma visão dos dados em qualquer domínio sob uma abordagem de classificação semi-supervisionada, permitindo melhorar o desempenho da classificação sem aumentar a quantidade de exemplos rotulados por especialistas. Para tal objetivo, utiliza-se do arcabouço teórico oferecido pelo Aprendizado Semi-supervisionado Baseado em Desacordo.

Portanto, neste trabalho pretende-se abordar tarefas de classificação semi-supervisionada de dados utilizando informações provenientes de diferentes estratégias para recuperar os $k$ vizinhos mais próximos de um exemplo. O algoritmo Co2KNN é proposto como alternativa mono-visão semi-supervisionada à abordagem multi-visão. Especificamente, se estudará o algoritmo proposto em domínios gerais nos quais uma matriz de distâncias entre os exemplos possa ser gerada. Com a matriz de distância disponível, propõe-se gerar 
dois classificadores baseados em duas estratégias diferentes para encontrar os vizinhos mais próximos de um exemplo. Uma primeira estratégia, chamada de KNN Local, utiliza o método tradicional K-Vizinhos Mais Próximos para predizer a classe de um exemplo não rotulado. Ou seja, recupera os $k$ vizinhos mais similares do exemplo e os utiliza para classificá-lo. A segunda estratégia, chamada de KNN Global, faz um caminho inverso. Ela recupera os exemplos que contém o novo exemplo dentre os seus $k$ vizinhos mais próximos. Com os dois classificadores gerados, começa a etapa de treinamento semi-supervisionado utilizando uma abordagem do estilo Cotraining. Finalmente, gera-se um classificador que combina as duas estratégias para rotular novos exemplos fora do conjunto de treino, sendo este classificador final o resultado do algoritmo Co2KNN.

A hipótese deste trabalho é que a combinação das duas estratégias já mencionadas no aprendizado semi-supervisionado significará uma melhora na classificação quando comparados com tais estratégias por separado e outras estratégias alternativas.

Neste trabalho, faz-se um estudo das duas estratégias de recuperação de vizinhos, mostrando os casos em que a estratégia KNN Global pode ajudar na classificação. Um dos problemas do KNN Global é a sua complexidade de tempo, que pode ser considerada inviável em vários domínios reais. Por tal motivo, neste trabalho propõe-se um algoritmo otimizado que permite utilizar tanto a estratégia KNN Global quanto o algoritmo Co2KNN.

Assim, as contribuições principais deste trabalho são: (1) a definição de duas estratégias de determinação de vizinhança de um exemplo; (2) definição de uma estratégia mono-visão do estilo Cotraining para a combinação do KNN Local e KNN Global; e (3) otimização do algoritmo para sua viabilidade em problemas reais.

\subsection{Organização do Trabalho}

Os próximos capítulos são estruturados da seguinte forma: no Capítulo 2 são apresentados os conceitos de aprendizado semi-supervisionada, com ênfase no algoritmo Cotraining e a aprendizado semi-supervisionada multi-visão; no Capítulo 3 são apresentados os conceitos da aprendizado baseado em instâncias, destacando-se as vantagens e desvantagens do classificador KNN; no Capítulo 4 são detalhadas as estratégias utilizadas neste trabalho e a abordagem de aprendizado semi-supervisionada de dados denominada Co2KNN; no Capítulo 5 são especificados os procedimentos para avaliação experimental e os resultados alcançados são apresentados e discutidos; e finalmente no Capítulo 6 são apresentadas as conclusões e trabalhos futuros. 


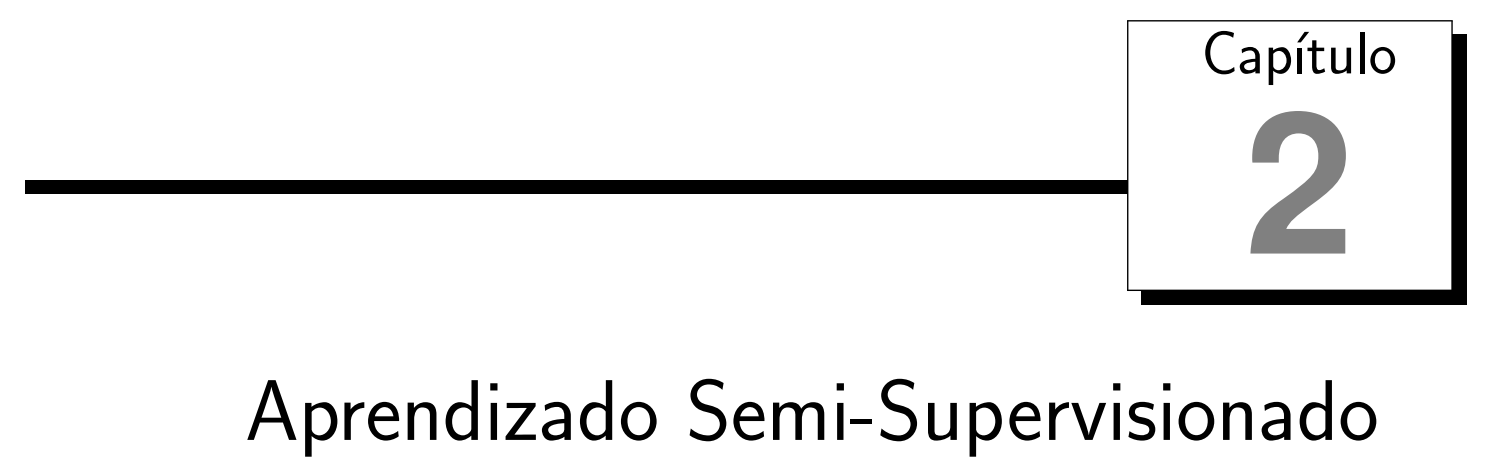

\subsection{Considerações Iniciais}

A Mineração de Dados (MD) visa extrair informação explícita e útil, previamente desconhecida a partir de uma coleção de dados (Witten e Frank, 2005). Claramente esta tarefa não é trivial, pois extrair essas informações pode significar procurar padrões específicos dentro de uma imensidade de padrões sem muita utilidade. Nesse cenário, a área de Aprendizado de Máquina (AM) provê um arcabouço teórico para o uso de diversos algoritmos e técnicas de extração automática de padrões que podem ser aplicadas à mineração de grandes coleções de dados (Mitchell e Blum, 1997). Os dados dessas coleções podem ser previamente preparadas por especialistas para diversas tarefas de AM, sendo possível encontrar bases de dados com alguns dados já classificados manualmente para o seu uso no processo de aprendizado. Estes dados são conhecidos como dados rotulados, sendo o restante conhecido como dados não rotulados.

O aprendizado semi-supervisionado posiciona-se entre as abordagens conhecidas como aprendizado supervisionado e aprendizado não supervisionado. O aprendizado supervisionado generaliza padrões presentes nos dados rotulados de treinamento para predizer alguma informação em novos dados não rotulados (Mitchell e Blum, 1997). Entretanto, o aprendizado não supervisionado aproveita os padrões interessantes presentes nos dados não rotulados para estimar a sua estrutura e extrair conhecimento da mesma (Barlow, 1999).

O Aprendizado Semi-Supervisionado é uma das técnicas de AM que aproveita os padrões contidos tanto nos dados rotulados quanto nos dados não rotulados. Devido a essa característica, o aprendizado semi-supervisionado tem emergido como uma alternativa interessante, especialmente quando uma grande quantidade de dados não rotulados está disponível e rotular os dados manualmente é muito custoso (Chapelle et al., 2006). 
Com o objetivo de entender melhor o funcionamento do aprendizado semi-supervisionado, tanto o aprendizado não supervisionado quanto o supervisionado são formalmente definidos e tratados com maior profundidade a seguir.

\subsection{Aprendizado Supervisionado e Não Supervisionado}

Na maioria dos cenários, os dados não se encontram no formato ideal para a utilização de algoritmos de AM. Dentro do AM existem representações específicas para os dados, para o conhecimento de fundo e para o modelo de aprendizado gerado (Russell e Norvig, 2003). Dentro dessas representações específicas, os algoritmos de AM necessitam que os dados sejam transformados em uma representação inteligível para o seu uso posterior por estes algoritmos. Tradicionalmente, a matriz atributo-valor é a representação mais utilizada devido a sua simplicidade, porém, necessita de um pré-processamento dos dados que possibilite a geração de tal representação. Por exemplo, na mineração de textos, cada documento pode ser representado por uma linha de uma tabela atributo-valor, sendo que cada termo existente na base é utilizado como atributo, e os valores podem ser representados com base na freqüência desses termos dentro do próprio texto (Feldman e Sanger, 2006).

Formalmente, no aprendizado não supervisionado, a representação dos valores dos atributos de um exemplo pode ser definida como $\vec{x}=\left(a_{1}, \ldots, a_{m}\right)$. Portanto, utiliza-se $X=\left(\overrightarrow{x_{1}}, \ldots, \overrightarrow{x_{n}}\right)$ para definir uma matriz $(n \times m)$ com os valores dos atributos que representam cada exemplo da base de dados. Nota-se que com esta representação vetorial pode-se considerar cada exemplo como se fosse um ponto no espaço euclidiano. Assume-se que cada exemplo $\overrightarrow{x_{i}}$ foi escolhido de "forma independente e com a mesma probabilidade" de alguma distribuição de dados desconhecida com densidade $\rho(x)$. De forma geral, o aprendizado não supervisionado visa estimar esta função de densidade sem a ajuda de algum supervisor que faça uma avaliação do erro cometido (Hastie et al., 2003).

Entre as principais técnicas de aprendizado não supervisionado pode-se citar as regras de associação (Agrawal et al., 1993), análise de agrupamento ou clustering (Kaufman e Rousseeuw, 1990), análise de componentes (Anderson, 1963; Comon, 1994), entre outras.

Por outro lado, no aprendizado supervisionado o objetivo é aprender novos conceitos dado um conjunto inicial de treinamento. O aprendizado desses novos conceitos podem ser representados como a estimação de uma função ou mapeamento de $\vec{x}$ para $y$, dado um conjunto inicial de treinamento constituído por $l$ pares do tipo $\left(\overrightarrow{x_{i}}, y_{i}\right)$, o conjunto de treinamento pode ser definido com un vetor da forma $X_{L}=\left\langle\overrightarrow{x_{i}}, y_{i}\right\rangle_{i=1}^{l}$. Neste cenário, a variável $y$ denota o conceito a ser aprendido (também conhecido como classe ou rótulo do exemplo). Portanto, o objetivo do aprendizado supervisionado é induzir uma função $f(\vec{x})=y$, a partir de uma série de exemplos com valores $y$ conhecidos. Neste caso, também se assume que os dados são escolhidos de "forma independente e com a mesma probabilidade" de alguma distribuição em $X \times Y$. Quando a saída é contínua, ou seja, 
$y \in \Re$, a tarefa é conhecida como regressão e quando a saída é discreta, ou seja, $Y=$ $\left\{y_{1}, \ldots y_{m}\right\}$, a tarefa é conhecida como classificação.

Dentro da classificação, a configuração mais simples é aquela onde a classe pode tomar somente dois valores, por exemplo \{masculino, feminino $\}$. Esta configuração é chamada de classificação binária e usualmente uma classe é definida como classe positiva e a outra como classe negativa.

As bases de dados podem ser muito grandes e, por isso, gerar classificadores que gerem hipóteses precisas para todos os cenários possíveis é uma tarefa muito complexa. Por esse motivo, existe uma variedade de métodos de inferência que fazem diferentes pressupostos sobre a distribuição dos dados, sob os quais garante-se um bom desempenho dos classificadores. Entre os principais métodos de inferência para classificação encontramse as árvores de decisão (Quinlan, 1986), o aprendizado baseado em regras (Furnkranz, 1999), as redes neurais (Bishop, 1995), o aprendizado baseado em instâncias (Aha et al., 1991), o aprendizado Bayesiano (Berger, 1985).

Por exemplo, as árvores de decisão pressupõem que existem atributos o suficientemente discriminativos para classificar os dados. Sendo assim, as árvores de decisão aproveitam tais atributos para separar os dados em subgrupos, procurando formar subgrupos que contenham apenas elementos de uma classe ou que a maioria dos elementos pertença a uma classe. Claramente, as árvores de decisão terão um melhor desempenho se tal pressuposto for cumprido.

Segundo Chapelle et al. (2006), existem duas grandes famílias de algoritmos supervisionados: os modelos gerativos e os modelos discriminativos (também conhecidos como modelos preditivos). Os modelos gerativos seguem a teoria clássica estatística para calcular a probabilidade condicional $P(x \mid y)$ por algum método não supervisionado. Nesse contexto, a função $f(\vec{x})=y$ pode ser definida como a probabilidade de $P(y \mid x)$, ou seja, a probabilidade de $y$ assumir o valor de alguma classe dado que o conjunto de valores dos atributos é conhecido previamente. Para este caso, a função retornará a classe que obtenha a maior probabilidade. Aplicando o teorema de Bayes, pode-se definir essa probabilidade com a equação 2.1 , sendo que $y^{\prime} \in\left\{y_{1}, \ldots, y_{m}\right\}$.

$$
P(y \mid x)=\frac{P(x \mid y) P(y)}{\sum_{y^{\prime}} P\left(x \mid y^{\prime}\right) \times P\left(y^{\prime}\right)}
$$

Estes tipos de algoritmos são chamados modelos gerativos devido ao fato que a probabilidade conjunta $P(x, y)=P(x \mid y) \times P(y)$ pode ser definida como a densidade que gera a distribuição dos dados, e seu cálculo é necessário na fórmula de $P(y \mid x)$. Um exemplo representativo deste tipo de algoritmo é o Expectation Maximization (Dempster et al., 1977), o qual se baseia na maximização da probabilidade conjunta $P(x, y)$ assumindo que a densidade de cada classe pode ser modelada por uma distribuição Gaussiana (ou seja, os exemplos se distribuem ao redor da média central). Em geral, os algoritmos gerativos tendem a fazer pressupostos fortes sobre a distribuição dos dados.

Por outro lado, os modelos discriminativos visam encontrar funções $f(\vec{x})$ que permitam 
discriminar entre exemplos de diferentes classes. Em outras palavras, não precisam fazer o cálculo da probabilidade $P(x \mid y)$, mas utilizam as propriedades da distribuição dos dados para induzirem o seu modelo. Estes métodos são conhecidos também como métodos preditivos, e são a base de muitos algoritmos do aprendizado de máquina. Por exemplo, na estrutura de trabalho das Máquinas de Vetores de Suporte (SVM), visa-se encontrar um hiperplano que divida os exemplos em classes diferentes, procurando maximizar a distância mínima de cada classe ao hiperplano (Vapnik, 1995). Nesse mesmo trabalho, Vapnik afirma que a desvantagem dos métodos gerativos está no cálculo de um problema mais geral, representado pelo cálculo da probabilidade $P(x \mid y)$, para o cálculo de um mais específico, sendo representado pela probabilidade $P(y \mid x)$.

Para qualquer modelo de aprendizado, assume-se ter disponível uma quantidade suficiente de exemplos rotulados para o treino dos classificadores. Porém, em muitos casos, a obtenção de dados rotulados é um processo muito custoso. Por exemplo, no problema da classificação de páginas Web, é necessário que um especialista leia a página visando atribuir um rótulo a mesma. Considerando um exemplo mais grave, para diagnosticar se uma pessoa está ou não com a gripe $A H 1 N 1$, o ideal seria que o médico tivesse o menor contato possível com pessoas doentes para não correr o risco de também infectar-se.

Frente a dificuldade de obter dados rotulados, emergiu a abordagem semi-supervisionada que aproveita os dados não rotulados junto com os dados rotulados para o aprendizado. Esta abordagem é detalhada a seguir.

\subsection{Aprendizado Semi-Supervisionado}

Como já comentado, o aprendizado semi-supervisionado se posiciona entre o aprendizado supervisionado e o aprendizado não supervisionado. Existem diversas formas de aproveitar os dados não rotulados para ajudar no aprendizado, tanto para o aprendizado supervisionado quanto para o não supervisionado. As duas estratégias mais estudadas no aprendizado semi-supervisionado são o agrupamento com restrições, mais relacionado com o aprendizado não supervisionado, e a classificação semi-supervisionada, mais relacionada com o aprendizado supervisionado.

O agrupamento com restrições é uma extensão do agrupamento não supervisionado (Abu-Mostafa, 1995). Além da informação tradicional dos atributos representados pela matriz $X=\left(\overrightarrow{x_{1}}, \ldots, \overrightarrow{x_{n}}\right)$, o conjunto de treinamento consiste em restrições da forma mustlink e cannot-link entre dois exemplos da base de dados. Enquanto a restrição cannot-link indica que dois exemplos $\overrightarrow{x_{i}}$ e $\overrightarrow{x_{j}}$ não podem estar no mesmo cluster a must-link indica que necessariamente aqueles exemplos pertencem ao mesmo cluster. Essas restrições podem ser consideradas como informação supervisionada sobre os clusters, pois são informações fornecidas por um especialista do domínio para ajudar na tarefa de agrupamento.

A classificação semi-supervisionada é uma extensão da classificação supervisionada, pois o conjunto de treinamento além de conter informações disponíveis pelos dados ro- 
tulados da forma $X_{L}=\left\langle\vec{x}_{i}, y_{i}\right\rangle_{i=1}^{l}$, utiliza-se de informações presentes nos dados não rotulados, sendo que estes podem ser representados da forma $X_{U}=\left\langle\overrightarrow{x_{j}}\right\rangle_{j=l+1}^{l+u}$. Nota-se que a quantidade total de dados de treinamento é $l+u$, sendo que um cenário propício é quando $u \gg l$. Entretanto, nada impede a utilização dos dados não rotulados em outros cenários.

Cozman e Cohen (2006) fizeram uma pesquisa sobre a utilização dos dados não rotulados para melhorar o desempenho de classificadores. Nessa pesquisa é mostrado que nem sempre é bom utilizar os dados não rotulados, pois é possível que os classificadores, em especial os classificadores gerativos, degradem o seu desempenho. Os autores atribuem as possíveis causas a utilização de modelos estatísticos errados para definir o comportamento dos dados não rotulados e afirmam que, nos casos em que existe uma quantidade suficiente de dados rotulados, deveria-se utilizar uma abordagem supervisionada sem ajuda dos dados não rotulados. Somente nos casos em que os classificadores supervisionados fossem muito fracos, deveria-se utilizar estratégias semi-supervisionadas. Singh et al. (2008) afirmam que existe uma grande quantidade de cenários nos quais a classificação semi-supervisionada pode até melhorar o desempenho da classificação supervisionada utilizando uma quantidade muito menor de dados rotulados de treinamento, sempre que certos pressupostos sejam cumpridos. Esta característica é comentada com mais detalhe a seguir.

\subsubsection{Pressupostos do Aprendizado Semi-Supervisionado}

Nem sempre é vantajoso utilizar dados não rotulados, por isso faz-se necessário estudar quando é possível combinar os dados rotulados e não rotulados e melhorar o processo de aprendizado. Apesar de nem todos os métodos serem probabilísticos, pode-se assumir que todos os métodos representam a sua hipótese como $P(y \mid x)$ e os dados não rotulados como $P(x)$. Como já mostrado no caso supervisionado, a densidade dos dados não rotulados $P(x)$ influencia na inferência da hipótese $P(y \mid x)$. Esta idéia, principalmente utilizada pelos métodos gerativos, foi criticada para o caso supervisionado, pois argumenta-se ser um trabalho a mais calcular essa densidade. No entanto, no caso semi-supervisionado é necessário aproveitar a distribuição dos dados não rotulados para ajudar na classificação. Com isso, pode-se definir que os dados não rotulados poderão ser utilizados para ajudar no processo de aprendizado quando $P(x)$ apresente informação útil para ajudar na classificação (Zhu, 2007).

Entretanto, como saber se $P(x)$ contém informações úteis para o processo de aprendizado? Não existe um método direto para responder essa questão, por esse motivo os métodos de aprendizado semi-supervisionado fazem alguns pressupostos sobre a relação entre a distribuição marginal $P(x)$ e a hipótese $P(y \mid x)$ (Zhu e Goldberg, 2009).

O primeiro é o pressuposto de suavidade ou Smoothness Assumption. Este pressuposto define que os rótulos dos exemplos devem variar de uma forma suave em áreas de densidade alta (Chapelle et al., 2006). Em outras palavras, se dois pontos $\overrightarrow{x 1}, \overrightarrow{x 2}$ ficarem próximos, 
suas saídas $y 1, y 2$ deveriam ser similares. Na Figura 2.1 são mostradas duas distribuições de dados com dois atributos $a 1$ e $a 2$, e duas classe $\times$ e $\mathbf{\square}$, sendo que a primeira cumpre com este pressuposto e a outra não cumpre.

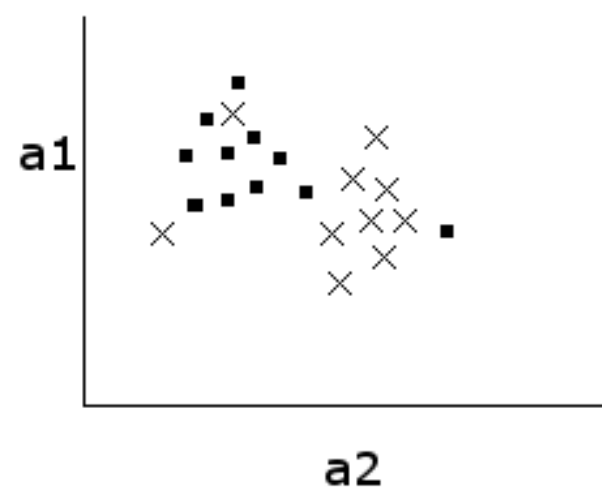

(a) Cumpre

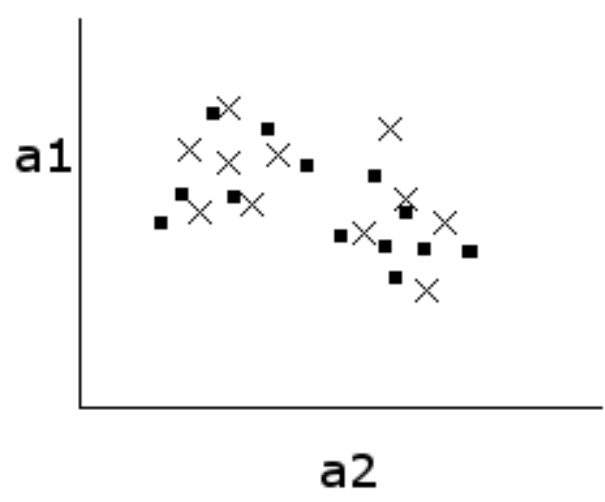

(b) Não cumpre

Figura 2.1: Pressuposto de suavidade.

O Segundo é o pressuposto de formação de grupos ou Cluster Assumption. Neste pressuposto assume-se que pontos que formam um mesmo grupo tendem a pertencer à mesma classe. Observa-se que se define somente uma tendência, permitindo a existência de exemplos que não cumprem este pressuposto com uma freqüência menor. Pode-se definir este pressuposto como uma extensão do pressuposto de suavidade, com a diferença que este pressuposto define que a formação de grupos implica áreas de separação de baixa densidade entre eles, o que não é explicitamente definido pelo pressuposto de suavidade. Na Figura 2.2a é mostrada uma distribuição que apresenta tanto a formação de grupos entre exemplos da mesma classe quanto a suavidade entre exemplos próximos. Já na Figura 2.2b é mostrada uma distribuição que apresenta o pressuposto de suavidade mas não apresenta o pressuposto de formação de grupos.

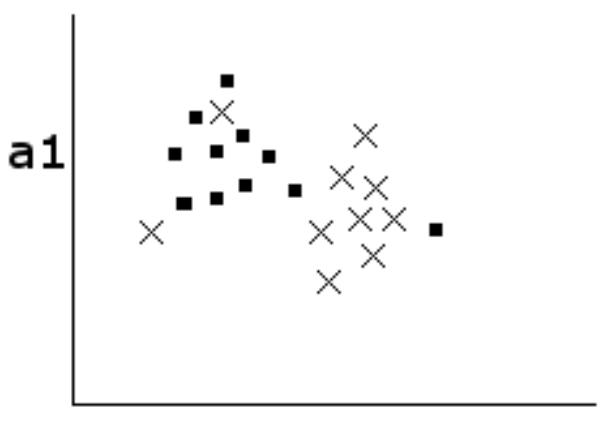

a2

(a) Cumpre

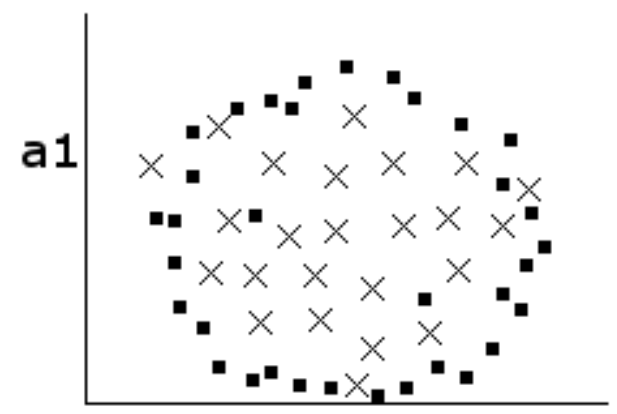

a2

Figura 2.2: Pressuposto de formação de grupos.

Dependendo do problema a tratar, o aprendizado semi-supervisionado pode-se dividir 
em duas estratégias: Aprendizado Indutivo e Aprendizado Transdutivo. Essas estratégias são apresentadas a seguir.

\subsubsection{Aprendizado Indutivo vs. Transdutivo}

Intuitivamente, o aprendizado semi-supervisionado visa utilizar os dados não rotulados disponíveis para melhorar a hipótese do classificador. Nesse contexto, dois cenários diferentes emergem dependendo do domínio de trabalho do classificador a ser gerado. $\mathrm{O}$ primeiro cenário é conhecido como aprendizado indutivo e tem como objetivo induzir um classificador que consiga rotular novos exemplos fora do conjunto de dados não rotulados disponíveis para treinamento. O segundo cenário é conhecido como aprendizado transdutivo e visa rotular os exemplos não rotulados conhecidos sem generalizar o modelo para novos dados desconhecidos. Em outras palavras, enquanto o aprendizado transdutivo conhece previamente todos os exemplos dos quais o rótulo precisa ser predito, o aprendizado indutivo não os conhece.

Portanto, dependendo da característica do problema, pode-se definir o problema a tratar e, com isso, definir se a estratégia semi-supervisionada a utilizar será transdutiva ou indutiva. Por exemplo, no problema da classificação on-line de e-mails em Spam e Não Spam, a geração de novos e-mails é um processo constante, sendo impossível a aplicação de métodos transdutivos para este problema específico. Entretanto, a classificação de uma série de pessoas como terrorista ou Não terrorista conhecidas previamente por serem suspeitas é um caso propício para a aplicação de métodos transdutivos.

Formalmente, o aprendizado indutivo treina o classificador utilizando o conjunto de treinamento que pode conter tanto dados rotulados $X_{L}$, quanto dados não rotulados $X_{U}$. Este tipo de aprendizado visa aprender uma função da forma $f: X \rightarrow Y$, de forma que $f$ obtenha um bom desempenho na predição de rótulos em exemplos novos fora de $X_{U}$.

Entretanto, tendo como entrada tanto os dados rotulados $X_{L}$, quanto os dados não rotulados $X_{U}$, o aprendizado transdutivo visa gerar uma função da forma $f: X^{l+u} \rightarrow$ $Y^{l+u}$, esperando-se que $f$ obtenha um bom desempenho na predição de rótulos de $X_{U}$.

O aprendizado transdutivo foi introduzido por Vapnik e Sterin (1977), sendo o mesmo autor quem definiu a filosofia da inferência transdutiva em Vapnik (1995). Segundo Vapnik, "para resolver um problema de interesse, não é necessário resolver um problema mais geral como passo intermediário", sendo este princípio a base da crítica dos modelos gerativos. Em outras palavras, dada uma série de exemplos rotulados e uma série de exemplos nos quais é necessário fazer uma predição dos rótulos, os métodos indutivos calculam uma função geral para todo o intervalo do universo de valores, enquanto os métodos transdutivos conseguem calcular uma função mais simples para todo o intervalo de $X_{U}$. Vapnik (2006) faz um estudo mais aprofundado sobre a relação entre o aprendizado transdutivo e o aprendizado semi-supervisionado.

O aprendizado semi-supervisionado tem ganho um interesse maior na comunidade científica desde a década dos anos 90. Com isso, diversos métodos semi-supervisionados 
foram desenvolvidos, tendo tanto estratégias apropriadas para o aprendizado indutivo quanto transdutivo. As principais técnicas semi-supervisionadas são apresentados a seguir.

\subsubsection{Técnicas do Aprendizado Semi-Supervisionado}

Cada método semi-supervisionado explora de uma forma mais específica algum dos pressupostos do aprendizado semi-supervisionado apresentados anteriormente e os estendem para aproveitar os dados não rotulados (Zhu e Goldberg, 2009). Dependendo da estratégia utilizada, é possível definir um esquema geral das técnicas do aprendizado semisupervisionado. Entre as principais técnicas estão o Selftraining (Scudder, 1965; Fralick, 1967), os modelos gerativos (McCallum e Nigam, 1998; Nigam e Ghani, 2000; Druck et al., 2007), o SVM semi-supervisionado (Vapnik, 1982, 1995), os métodos baseados em grafos (Zhu, 2005) e o aprendizado semi-supervisionado multi-visão (Blum e Mitchell, 1998).

\section{Selftraining}

O Selftraining é uma técnica tradicional caracterizado por ter uma estrutura de aprendizado simples. A idéia básica do Selftraining é aprender das próprias predições utilizando o processo chamado de "Bootstrapping". Neste processo, utilizam-se os rótulos preditos para re-treinar o classificador e modificar a hipótese (Abney, 2002), e o Selftraining aproveita este conceito para re-treinar o classificador iterativamente. Como mostrado no Algoritmo 1, o Selftraining em cada iteração treina o classificador com conjunto de dados rotulados $L$, rotula o conjunto de exemplos não rotulados $U$, escolhe um subconjunto $S$ dos dados não rotulados com suas respectivas classes preditas para incrementar o conjunto $L$. Geralmente $S$ representa o conjunto dos exemplos rotulados com a maior confiança pelo classificador. O algoritmo termina quando o conjunto $U$ estiver vazio ou atingir algum outro critério de parada. O principal pressuposto que o algoritmo Selftraining faz é que os rótulos preditos em cada iteração, pelo menos os escolhidos dentro do conjunto $S$, são corretos. Este pressuposto está ligado com o pressuposto de formação de grupos, pois aqueles exemplos rotulados com a maior confiança serão aqueles que têm a maior similaridade com os exemplos do conjunto $U$ (Zhu e Goldberg, 2009).

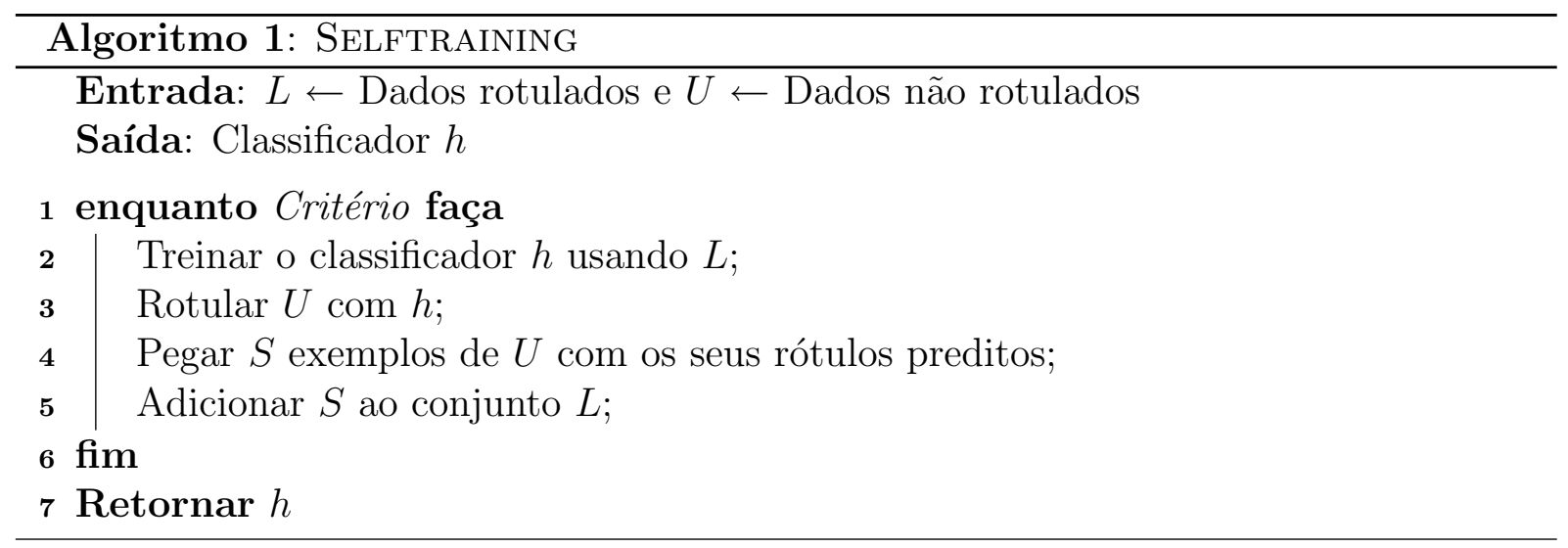


O algoritmo Selftraining é um algoritmo Wrapper, ou seja, não fixa o tipo de classificador utilizado. Poderia ser desde um simples classificador baseado nos $K$ vizinhos mais próximos até uma rede neural. Por este motivo, este algoritmo pode ser utilizado para estratégias tanto indutivas quanto transdutivas.

\section{Modelos Gerativos}

De maneira similar com a abordagem gerativa supervisionada, os modelos gerativos são uma abordagem estatística que utiliza o modelo $P(x, y)=P(x \mid y) P(y)$ para fazer o cálculo da hipótese $P(y \mid x)$. A idéia deste modelo é que inicialmente a distribuição conjunta de todas as classes é conhecida e que é possível decompor a distribuição conjunta em distribuições individuais (Zhu e Goldberg, 2009). Esta idéia pode ser definida como uma extensão do pressuposto de suavidade do aprendizado semi-supervisionado. O algoritmo mais representativo deste modelo é o Expectation Maximization (EM), que pressupõe que os exemplos de cada classe seguem uma distribuição Gaussiana. Com uma grande quantidade de dados não rotulados, os componentes e parâmetros dessas distribuições Gaussianas podem ser calculados, modificando-os iterativamente com o objetivo de maximizar a verossimilhança entre os exemplos da mesma distribuição. Por exemplo, na Figura 2.3 é mostrada uma mesma distribuição para duas etapas diferentes do algoritmo EM. Inicialmente o algoritmo EM pode estimar que as classes dos exemplos distribuem-se conforme ilustrado na Figura 2.3a. Após algumas iterações, o algoritmo modifica os parâmetros das distribuições preditas, chegando nas distribuições da Figura 2.3b.

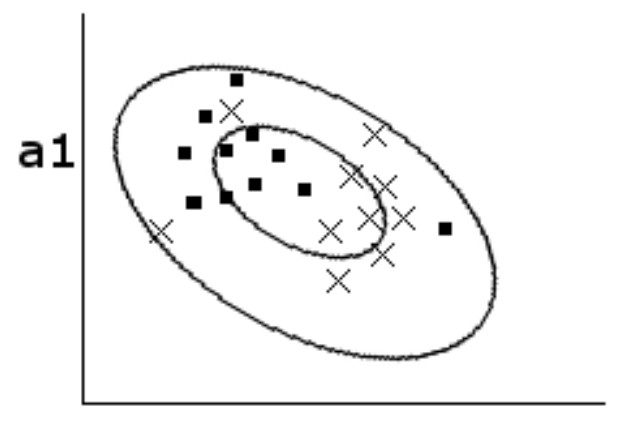

a2

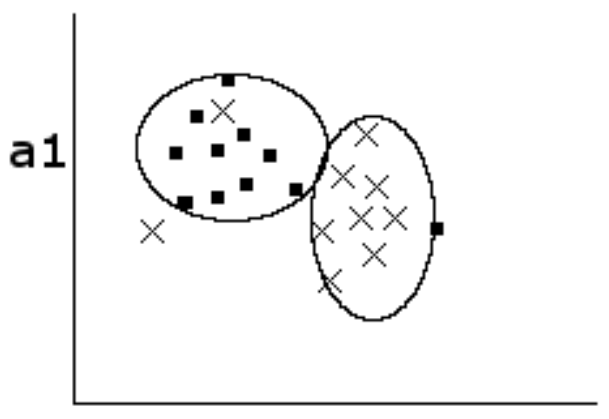

a2

(a) Inicial

(b) Final

Figura 2.3: Predição da distribuição das classes com o algoritmo EM

Por esse motivo, o algoritmo EM já foi bastante utilizado na abordagem semi-supervisionada. Entre os trabalhos mais representativos podem-se citar a Nigam e Ghani (2000); Rayid (2001) e Cong et al. (2004), que aplicam o algoritmo EM na tarefa de classificação de textos, obtendo bons resultados devido ao fato que a representação em tabelas atributo-valor baseadas nas freqüências das palavras, também conhecida como bagof-words, leva a formação de modelos que cumprem os pressupostos sobre a distribuição dos dados. Moreno e Agarwal (2003) aplicam o algoritmo EM semi-supervisionado em 
dados de audio tendo importantes resultados neste domínio frente à grande dificuldade de anotar e rotular os arquivos de áudio manualmente. Para um estudo mais detalhado dos métodos gerativos e do algoritmo EM podem-se referenciar a Dempster et al. (1977); Mclachlan e Krishnan (1996); McCallum e Nigam (1998); Dellaert (2002) e Hastie et al. (2003).

\section{SVM semi-supervisionado}

O interesse pelo aprendizado semi-supervisionado cresceu nos finais da década de 90, especialmente em problemas de linguagem natural e classificação de textos (Bennett e Demiriz, 1998; Blum e Mitchell, 1998; Mccallum e Mitchell, 2000). Um dos algoritmos propostos para trabalhar com poucos dados rotulados foi a versão semi-supervisionada SVM (Vapnik, 1982, 1995). O algoritmo SVM procura um hiperplano que divida os exemplos de cada classe, procurando maximizar a distância mínima de cada grupo de exemplos com o hiperplano. No método supervisionado, considerava-se somente exemplos rotulados nesse processo e utilizava-se otimização de funções para encontrar o hiperplano. A versão semi-supervisionada adiciona a informação da distribuição dos dados $P(x)$ para conseguir melhorar a hipótese do classificador, fazendo o pressuposto que existe uma região de baixa densidade que separa os exemplos de diferentes classes. Este pressuposto pode ser considerado uma extensão do pressuposto de existência de grupos. Por exemplo, na Figura 2.4 é mostrado o hiperplano gerado pela SVM em dois cenários distintos. O primeiro cenário, ilustrado na Figura 2.4a, utiliza somente os dados rotulados para gerar a hipótese do classificador. Já na Figura 2.4b, o algoritmo SVM aproveita os dados não rotulados para melhorar a hipótese e conseguir discriminar os exemplos com uma maior acurácia.

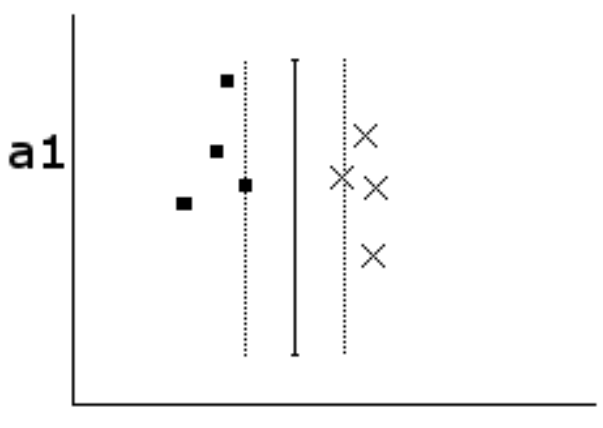

a2

(a) Supervisionado

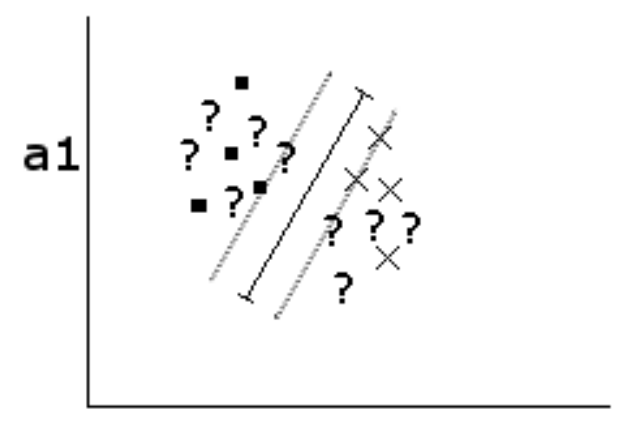

a2

(b) Semi-supervisionado

Figura 2.4: Hiperplanos gerados pela SVM para separar as classes. Figura adaptada de Zhu e Goldberg (2009).

Inicialmente, esta abordagem foi chamado SVM transdutivo, pois o objetivo inicial foi gerar um classificador eficiente para os dados não rotulados disponíveis (Vapnik, 1995; Joachims, 1998, 1999). Contudo, com a mesma hipótese gerada é possível rotular novos 
dados além dos disponíveis para o treino, o que enfatiza que o nome de SVM semisupervisionado é mais coerente (Bennett e Demiriz, 1998; Zhu e Goldberg, 2009).

\section{Métodos Baseados em Grafos}

Tipicamente, os métodos baseados em grafos assumem que os dados formam grandes coleções contínuas em alguma dimensão, formando curvas hiper-dimensionais. Este pressuposto é conhecido como o pressuposto de geração de coleções ou manifold assumption (Chapelle et al., 2006), e os grafos de similaridade tem grande capacidade de modelagem dos dados quando este pressuposto é cumprido. Claramente, a manifold assumption é uma extensão do pressuposto de agrupamentos. A idéia destes métodos é construir grafos nos quais os vértices definem os exemplos da base de dados (tanto os rotulados quanto os não rotulados) e as arestas representam a similaridade entre os mesmos. Os grafos gerados podem ser completos, tendo $v$ vértices e $v *(v-1) / 2$ arestas, ou podem ser redes nas quais cada vértice tem uma aresta com os seus $k$ vizinhos mais próximos. Na Figura 2.5 é mostrado um exemplo para um grafo formado pelos 3 vizinhos mais próximos. Deve-se notar que no caso da formação de grafos dos $k$ vizinhos, é possível que um vértice tenha mais de $k$ arestas.

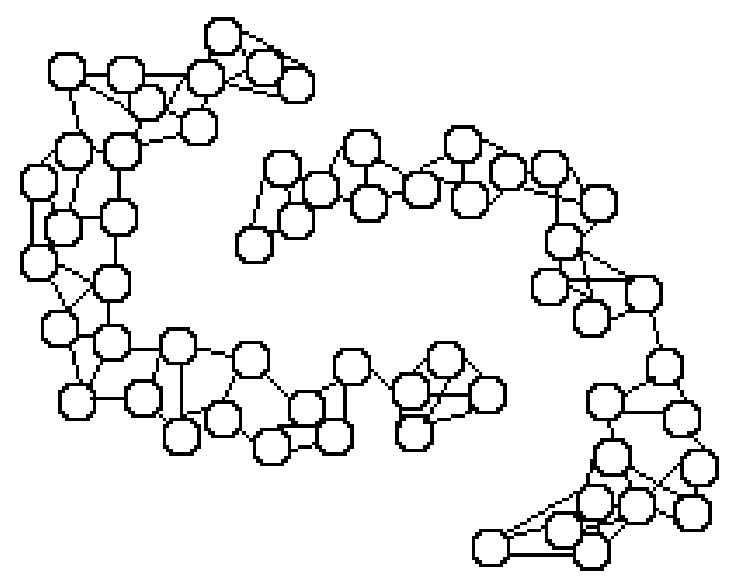

Figura 2.5: Grafo formado pelos 3 vizinhos mais próximos de cada vértice.

Após o processo de geração do grafo, a maior parte dos algoritmos dividem os grafos em componentes (conjunto de vértices no qual para qualquer par de vértices existe um caminho) com os mesmos rótulos utilizando estratégias diversas para propagar os rótulos. Estas estratégias são geralmente transdutivas, pois após a predição dos rótulos dos vértices não rotulados o processo termina. Ou seja, inicialmente o grafo de similaridade é gerado, tanto com os dados rotulados quanto com os dados não rotulados, e posteriormente rotulase todos os vértices não rotulados do grafo com base em algum método de inferência. Por esse motivo, a adição de novos exemplos geralmente não é um cenário contemplado por estes algoritmos.

Além do manifold assumption, um outro pressuposto base para o sucesso destes algoritmos é que os rótulos mudam suavemente em relação ao grafo (Zhu e Goldberg, 2009), o 
qual pode ser considerado como uma extensão do pressuposto de suavidade. Ressalta-se que os métodos baseados em grafos são transdutivos por natureza, pois pressupõem que o grafo já está formado, tanto com vértices rotulados quanto com vértices não rotulados, para aplicar as técnicas adequadas. Geralmente, tais técnicas não são generalizáveis à rotulação de novos exemplos que podem aparecer posteriormente.

Segundo Zhu (2005), os métodos baseados em grafos visam estimar uma função $f$ que satisfaça duas restrições: deve concordar em grande parte com os rótulos dos vértices rotulados e deve cumprir o pressuposto de suavidade no grafo. A primeira restrição é conhecida como função de perda e a segunda a regularizadora. Diferentes métodos diferem na escolha das funções de perda e regularizadora, porém, o autor afirma que mais importante é a construção de um grafo de boa qualidade informativa.

Entre os principais algoritmos transdutivos baseados em grafos pode-se citar o algoritmo do Corte Mínimo ou Mincut (Blum e Chawla, 2001; Blum et al., 2004), que visa gerar componentes no grafo de similaridade, tal que instâncias similares contenham valores altos nos pesos das arestas. O algoritmo baseia-se na técnica do corte mínimo em grafos com peso, que visa encontrar a mínima quantidade de arestas que devem ser retiradas da rede para separá-la em componentes. Finalmente, classifica-se os elementos não-rotulados de cada componente com o valor do rótulo majoritário.

Outras abordagens visam predizer o rótulo de um exemplo novo utilizando-se dos rótulos da vizinhança para a inferência. Por exemplo, a Função Harmônica (Zhu et al., 2003) prediz o rótulo de um vértice $v_{i}$ calculando a média harmônica dos rótulos da vizinhança, utilizando o peso das arestas para ponderar a função. Cita-se também o algoritmo de Propagação de Rótulo (Zhu e Ghahramani, 2002), que é um algoritmo que utiliza os conceitos da função harmônica para propagar rótulos entre os componentes, sendo que aqueles vértices que tem a maior probabilidade de pertencer a uma classe são rotulados. O grafo é atualizado em um processo iterativo que tem como objetivo rotular o grafo completamente.

Apesar de que os métodos baseados em grafos são transdutivos por natureza, uma abordagem indutiva foi proposta. A Manifold Regularization utiliza o grafo de Laplace (Merris, 1998) em conjunto com outras funções espectrais de grafos para generalizar a função $f: X \rightarrow \Re$ e conseguir rotular exemplos novos. Entre os trabalhos que estudam mais aprofundadamente esta abordagem podem-se citar Chapelle et al. (2003); Sindhwani et al. (2005a) e Belkin et al. (2006). Para mais detalhes sobre os métodos semi-supervisionados baseados em grafos recomenda-se o trabalho de Zhu (2005).

Os grafos também podem conter relações explícitas nos domínios relacionais, permitindo gerar arestas diferentes da similaridade. Nestes domínios é comum a utilização da inferência coletiva para rotular os dados. Inferência coletiva significa inferir simultaneamente valores inter-relacionados dentro do grafo, calculando distribuições de probabilidades locais a cada vértice. Estes são processos iterativos que terminam quando as probabilidades locais convergem em valores únicos. Entre os trabalhos mais representa- 
tivos da inferência coletiva pode-se citar à Amostragem de Gibss (Geman e Geman, 1990), Suavização de Rótulos (Chakrabarti et al., 1998), Classificação Iterativa (Lu e Getoor, 2003), entre outros. A inferência coletiva é uma abordagem de classificação transdutiva em grafos que poderia ser utilizada tanto no aprendizado supervisionado quanto no semi-supervisionado.

\section{Aprendizado Multi-visão}

O objetivo principal do aprendizado semi-supervisionado é melhorar o desempenho dos classificadores utilizando as informações presentes nos dados não rotulados. Porém, a pouca quantidade de dados rotulados é ainda um problema que influencia no aprendizado dos classificadores. Nesse contexto, o aprendizado semi-supervisionado multi-visão surgiu com o objetivo de adicionar informação às representações tradicionais sem aumentar a quantidade de exemplos rotulados necessária para o treinamento dos classificadores.

A abordagem multi-visão foi inicialmente formalizada por Blum e Mitchell (1998), aproveitando a redundância de atributos obtidas por duas visões pouco correlacionadas. No entanto, a idéia de combinar diferentes fontes de informação já tinha sido explorada anteriormente. Sa (1993) começou a utilizar diferentes sensores de som para obter duas visões dos dados no processo de reconhecimento de vogais. Yarowsky (1995) enfrentou o problema da desambiguação do sentido de palavras contidas em documentos, utilizando tanto a informação geral dos documentos quanto o contexto local das palavras. A seguir é mostrado uma definição formal do aprendizado multi-visão.

Suponha-se que cada exemplo da base de dados é representado por $v$ visões diferentes. O espaço de instâncias é definido por $X=X^{(1)} \times X^{(2)} \times \ldots X^{(v)}$. Assumindo uma classificação binária por simplicidade, define-se $\left\{C_{1}, C_{2}\right\}$ como os rótulos dos exemplos. Nesse sentido, um exemplo pode ter diferentes classes preditas com diferente confiança para cada visão, da forma $C_{i}=f\left(\vec{x}^{(i)}\right)$, sendo que os algoritmos de aprendizado multi-visão combinam as diversas confianças na classificação para rotular o exemplo de uma forma mais precisa.

Entre os principais algoritmos do aprendizado semi-supervisionado multi-visão pode-se citar o algoritmo Cotraining (Blum e Mitchell, 1998), que propõe o uso de duas representações dos dados para gerar dois classificadores que consigam se ajudar mutuamente em um processo conhecido como bootstrapping (já comentado na explicação do algoritmo Selftrainig). O algoritmo Co-EM proposto por Nigam e Ghani (2000) é uma variação do algoritmo semi-supervisionado EM para aceitar mais de uma visão dos dados. O Coregularization (Sindhwani et al., 2005b), aproveita as múltiplas visões para fazer um melhor cálculo do hiperplano da versão semi-supervisionada do algoritmo SVM (Bennett e Demiriz, 1998). O OLTV (One Labeled example and Two Views), que necessita de duas visões dos dados e somente um exemplo rotulado, aproveitando a correlação entre elas para classificar exemplos não rotulados (Zhou et al., 2007).

Pesquisas recentes colocam o aprendizado semi-supervisionado multi-visão dentro de 
uma técnica ainda maior chamada Aprendizado Semi-supervisionado baseado em Desacordo (Li e Ogihara, 2005; Zhou, 2009; Zhou e Li, 2009). Esta técnica estuda a geração de diversos classificadores para um processo colaborativo no qual o desacordo na classificação de um elemento é explorado durante o aprendizado. Zhou e Li (2009) explicam que os dados não rotulados servem de plataforma para a troca de informações entre os classificadores, sendo que possivelmente um classificador tenha mais confiança na classificação de um exemplo e os outros aprendam este conceito para melhorar suas hipóteses. Se os classificadores aprendem usando diferentes visões, o cenário é considerado de aprendizado semi-supervisionado multi-visão. Se os classificadores são gerados sob uma mesma visão, o cenário é chamado de aprendizado semi-supervisionado mono-visão com múltiplos classificadores.

O algoritmo Cotraining é um dos algoritmos tradicionais do aprendizado semi-supervisionado e é a base do presente trabalho. Por esse motivo, será apresentado com maior detalhe a seguir.

\subsection{O Cotraining}

Blum e Mitchell propuseram utilizar a redundância de atributos obtida por duas visões diferentes dos dados para gerar dois classificadores capazes de aprender com exemplos rotulados iterativamente, a partir de um processo de bootstrapping conjunto. Blum e Mitchell avaliaram o algoritmo na classificação de páginas Web. A primeira visão é baseada nos textos de cada página, e a segunda visão é baseada nos textos dos hyperlinks que apontam a cada página. O Cotraining baseia-se na idéia de que um classificador rotulará exemplos que conterão informações desconhecidas pelo outro classificador, permitindo o processo de aprendizagem.

Formalmente, o modelo de aprendizado do Cotraining é definido da seguinte forma. $\mathrm{O}$ espaço de instâncias com duas visões que representam os dados é definido por $X=X^{(1)} \times$ $X^{(2)}$ sobre uma distribuição dos dados $D$. Com isso, o conjunto de exemplos rotulados é definido como $X_{L}=\left\langle{\overrightarrow{x_{i}}}^{(1)},{\overrightarrow{x_{i}}}^{(2)}, y_{i}\right\rangle_{i=1}^{l}$, e o conjunto de exemplos não rotulados é definido como $X_{U}=\left\langle{\overrightarrow{x_{j}}}^{(1)},{\overrightarrow{x_{j}}}^{(2)}\right\rangle_{j=l+1}^{l+u}$, assumindo que $u \gg l$. Dois classificadores $C_{1}$ e $C_{2}$ são treinados com o conjunto $X_{L}$, gerando duas hipóteses $h_{1}\left(X^{(1)}\right)$ e $h_{2}\left(X^{(2)}\right)$ sobre as funções objetivo respectivas $f_{1}\left(X^{(1)}\right)$ e $f_{2}\left(X^{(2)}\right)$. O Cotraining trabalha com o conceito de funções compatíveis, o qual se define da seguinte forma $f_{1}\left(X^{(1)}\right)=f_{2}\left(X^{(2)}\right)=y$. Em outras palavras, o modelo de aprendizado Cotraining assume que as funções objetivo geradas com as diferentes visões devem concordar na predição das classes, e devem ser corretas. Nesse sentido, espera-se que $h 1 \approx f_{1} \wedge h_{2} \approx f_{2}$, e, portanto, espera-se também que as hipóteses $h_{1}$ e $h_{2}$ concordem na predição da maior parte dos exemplos.

Para assegurar o conceito de compatibilidade entre as funções objetivo, o algoritmo Cotraining considera alguns pressupostos sobre a distribuição dos dados, apresentados a seguir. 


\subsubsection{Pressupostos do Cotraining}

O primeiro pressuposto define que cada visão é suficiente para treinar um classificador com bom desempenho caso a função de classificação respectiva seja conhecida e tenha-se uma quantidade suficiente de dados de treinamento. Este pressuposto é conhecido como o pressuposto de suficiência, pois cada classificador é suficiente para predizer a função objetivo (Ling et al., 2009).

O segundo pressuposto indica que as visões são condicionalmente independentes dada a classe. Em outras palavras, a representação obtida por uma visão não depende ou tem correlação direta com a outra visão. Este pressuposto é conhecido como o pressuposto de independência (Zhou e Li, 2009). Nota-se que, se as informações obtidas pelas duas visões são as mesmas, o modelo de aprendizado Cotraining se degrada para o modelo Selftraining.

Alguns resultados teóricos têm mostrado que quando os pressupostos são cumpridos, o algoritmo Cotraining garante um bom desempenho dos classificadores treinados (Nigam e Ghani, 2000; Dasgupta et al., 2002; Balcan et al., 2005). Porém, o pressuposto de independência é considerado muito rigoroso, devido ao fato que em um cenário real, a obtenção de dois conjuntos de atributos que representam duas visões reduz bastante a quantidade de domínios cobertos, e adicionar o pressuposto de independência torna muito difícil a aplicação deste algoritmo em problemas reais (Wang e Zhou, 2007). De fato, o pressuposto de independência é tão forte que, quando cumprido, é necessário que se tenha somente um exemplo para permitir um aprendizado com sucesso. Tal afirmação foi apresentada por Zhou et al. (2007), que mostraram que se houver disponível somente um exemplo positivo rotulado, pode-se encontrar o conjunto de atributos que mais identificam aquele exemplo e, portanto, encontrar o conjunto de exemplos que são mais correlacionados com o único exemplo rotulado, sendo também rotulados como positivos.

Por esse motivo, diversos trabalhos suavizaram o pressuposto de independência para abranger domínios nos quais as visões dos dados não são totalmente independentes obtendo resultados promissores (Nigam e Ghani, 2000; Goldman e Zhou, 2000; Abney, 2002; Zhou et al., 2007; Zhang e Zheng, 2009). O fator comum entre todos esses trabalhos é o fato de utilizarem duas visões que não são totalmente independentes.

\subsubsection{O Algoritmo Cotraining}

O Cotraining, mostrado no Algoritmo 2, supõe que inicialmente existem dois conjuntos de exemplos: o conjunto inicial de dados rotulados chamado $L$ e o conjunto de dados não rotulados $U$, os quais formam o conjunto de treinamento e são dados como entrada do algoritmo. Assim, a entrada do algoritmo é formada pelos conjuntos $L, U$ e as variáveis loops, que representa a quantidade de iterações que o algoritmo será executado e $p$ e $n$ que representam a quantidade de exemplos positivos e negativos, respectivamente, escolhidos em cada iteração por cada classificador. No primeiro passo, deve-se gerar um conjunto 
de exemplos não-rotulados $A$, com um tamanho menor do que $U$. O objetivo da geração deste conjunto será explicado posteriormente. Observa-se que os valores de loops não devem ser elevados para evitar a falta de exemplos de treinamento não-rotulados das classes minoritárias após várias iterações. Para cada iteração, cada classificador deve treinar usando a visão respectiva de cada exemplo do conjunto $L=\left\langle{\overrightarrow{x_{i}}}^{(1)},{\overrightarrow{x_{i}}}^{(2)}, y_{i}\right\rangle_{i=1}^{l}$. Após o treinamento, cada classificador deve escolher $p$ exemplos positivos classificados com maior confiança e $n$ exemplos negativos classificados com maior confiança. Em cada iteração, portanto, são rotulados $2 n+2 p$ exemplos, os quais são extraídos do conjunto $A$ e utilizados para atualizar o conjunto $L$. A seguir, deve-se repor os exemplos extraídos de $A$, escolhendo aleatoriamente $2 n+2 p$ exemplos não-rotulados de $U$. O uso do conjunto especial $A$ tem a função de manter a distribuição inicial dos dados não-rotulados durante todo o processo. Segundo Blum e Mitchell (1998), o uso desse conjunto especial força aos classificadores a selecionar exemplos que são mais representativos da distribuição dos dados em $U$. Finalmente, após loops iterações tem-se como saída do algoritmo dois classificadores com suas respectivas hipóteses $h 1$ e $h 2$, treinados semi-supervisionadamente. É possível gerar um terceiro classificador multiplicando as probabilidades de cada classificador para rotular novos dados, obtendo um novo valor de confiança no processo de classificação e, portanto, gerar um novo classificador chamado de classificador combinado (Blum e Mitchell, 1998).

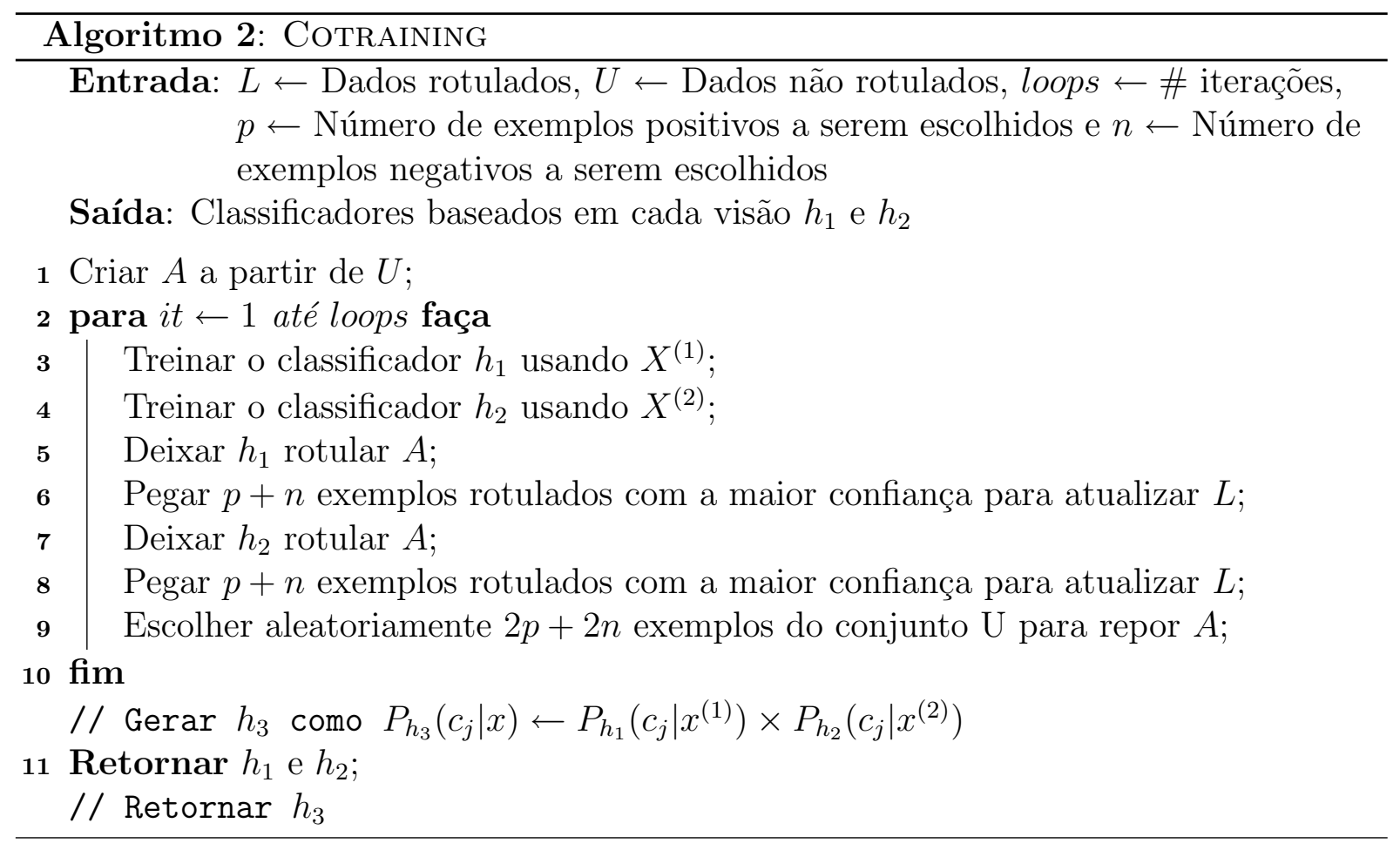

Algumas variações do algoritmo podem reduzir a dependência pela grande quantidade de parâmetros necessários, por exemplo, substituir os parâmetros $n$ e $p$ por um limiar de confiança para a seleção dos exemplos, ou iterar o algoritmo até não conseguir exemplos rotulados com confiança alta ou até o conjunto $U$ ficar vazio. Por esse motivo, denomina-se a estrutura geral do algoritmo como Família de Algoritmos Cotraining (Zhu e Goldberg, 
2009).

Sob o processo de aprendizado do algoritmo Cotraining, os exemplos rotulados por um classificador são utilizados como exemplos de treinamento pelo outro classificador, criando um processo de Bootstrapping em equipe. O fato de escolher os exemplos rotulados com a maior confiança para atualizar o conjunto $L$, conjuntamente com os pressupostos do algoritmos, permite que os classificadores aprendam um com o outro. Nesse sentido, exemplos em que um classificador tem confiança alta para rotulá-lo, e outro classificador tem uma confiança baixa são ótimos para este processo de aprendizado.

\subsubsection{O Cotraining e o Aprendizado Baseado em Desacordo}

Como já comentado, Blum e Mitchell (1998) definem o conceito de compatibilidade entre funções para mostrar o sucesso do algoritmo Cotraining. Dadas as duas visões $X^{(1)}$ e $X^{(2)}$, as funções compatíveis são definidas como $f_{1}\left(X^{(1)}\right)=f_{2}\left(X^{(2)}\right)=y$. Em outras palavras, $f=\left(f_{1}, f_{2}\right)$ é compatível com a distribuição $D$ se $D$ atribui probabilidade zero a qualquer exemplo $x=\left(x^{(1)}, x^{(2)}\right)$ no qual $f_{1}\left(x^{(1)}\right) \neq f_{2}\left(x^{(2)}\right)$.

Tal compatibilidade pode ser satisfeita mesmo que as distribuições verdadeiras das classes sejam muito complexas, e as funções $f_{1}$ e $f_{2}$ sejam menores e mais simples. Sendo tal compatibilidade satisfeita, os autores mostram que os dados não rotulados podem ser utilizados para diminuir a necessidade de dados rotulados necessários. Os autores explicam o conceito de compatibilidade como um processo de aprendizado rotativo em um grafo bipartido ${ }^{1}$. De um lado colocam-se os exemplos com a visão $X^{(1)}$, e do outro, o mesmos exemplos com a visão $X^{(2)}$. Nota-se que os vértices que estão um na frente do outro representam a duas visões para um mesmo exemplo. Existe uma aresta $\left(\overrightarrow{x_{1}}, \overrightarrow{x_{2}}\right)$ se a partir do conceito aprendido em $\overrightarrow{x_{1}}$ por um classificador é possível predizer o rótulo de $\overrightarrow{x_{2}}$ com uma probabilidade maior do que zero. As arestas de cor mais escura representam confianças altas para a classificação de um exemplo e as mais claras representam confianças baixas. O peso da aresta $\left(\overrightarrow{x_{1}}, \overrightarrow{x_{2}}\right)$ é proporcional à confiança com a qual o rótulo é predito. Na Figura 2.6 é mostrado um exemplo de grafo bipartido para uma iteração do Cotraining. Ressalta-se que, apesar de não se conhecerem os rótulos dos exemplos, pode-se observar a formação de três componentes, tendo dois deles probabilidades muito altas de conter elementos da mesma classe.

Claramente, se as funções $f_{1}$ e $f_{2}$ são compatíveis com a distribuição $D$, cada componente do grafo conterá exemplos de uma mesma classe. Em outras palavras, o conceito de compatibilidade indica que não pode existir uma aresta com um peso alto (probabilidade alta) que una dois exemplos de classes diferentes. O conceito de funções compatíveis são muito importantes para o algoritmo Cotraining, pois se arestas erradas fossem adicionadas no grafo, os classificadores aprenderiam exemplo rotulados erroneamente, e o desempenho dos classificadores se degradaria. Por esse motivo, o Cotraining faz pressupostos fortes

\footnotetext{
${ }^{1}$ Grafo bipartido é um grafo com dois subconjuntos de vértices, no qual não há arestas entre vértices do mesmo subconjunto.
} 


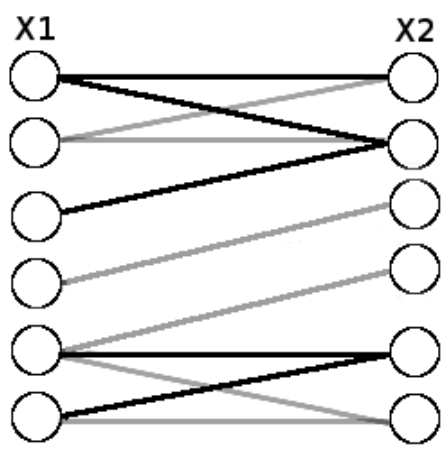

Figura 2.6: Grafo bipartido. Imagem modificada de Blum e Mitchell (1998).

sobre a distribuição e representações dos dados para assegurar um bom desempenho.

Balcan et al. (2005) afirmam que é possível suavizar ou até não precisar do pressuposto de independência, sempre que os dados cumpram o pressuposto de expansão, que é menos restritivo do que o pressuposto de independência. O pressuposto de expansão indica a probabilidade de que os classificadores rotulem com confiança alta os exemplos somente em uma das duas visões é maior ou igual do que os classificadores concordarem na classificação. Formalmente, se $S_{1} \subseteq X^{(1)} \wedge S_{2} \subseteq X^{(2)}$, e definimos $S_{1}$ e $S_{2}$ como o conjunto de exemplos nos quais cada visão tem uma confiança alta na classificação, então $P\left(S_{1} \wedge S_{2}\right)$ denota a probabilidade dos classificadores terem confiança alta nas duas visões, $P\left(S_{1} \oplus S_{2}\right)$ denota a probabilidade de somente uma visão ter confiança alta e $P\left(\bar{S}_{1} \wedge \bar{S}_{2}\right)$ representa a densidade de exemplos nos quais nenhuma das duas visões tem confiança alta. Com isso $D^{+}$é $\epsilon$-expansível se a probabilidade de somente uma visão ter confiança alta é maior ou igual do que as duas terem confiança alta ou as duas terem confiança baixa, como mostrado na Equação 2.2. Com o pressuposto de expansão, os autores mostram que os algoritmos do estilo Cotraining conseguem ter sucesso no treinamento semi-supervisionado de classificadores baseados em diferentes visões, mesmo se não são independentes.

$$
P\left(S_{1} \oplus S_{2}\right) \geq \epsilon \min \left[P\left(S_{1} \wedge S_{2}\right), P\left(\bar{S}_{1} \wedge \bar{S}_{2}\right)\right]
$$

Zhou e Li (2007) utilizam como base os estudos de Balcan et al. (2005), para apresentar um estudo teórico sobre o aprendizado baseado em desacordo. Como já comentado, o aprendizado baseado em desacordo indica que é possível combinar classificadores para se ajudarem mutuamente se eles discordarem na classificação de uma parte significativa dos exemplos, o que explica o sucesso do algoritmo Cotraining mesmo quando uma única visão nos atributos está disponível (Zhou e Li, 2009). Sendo definido $h^{*}$ como a hipóteses verdadeira da distribuição dos dados, $d\left(h, h^{*}\right)$ como a diferença entre uma hipótese $h$ e a hipótese verdadeira, e $h_{j}^{(i)}$ como a hipótese da visão $i$ na iteração $j$ do algoritmo Cotraining, o principal resultado do trabalho de Zhou e Li (2007) é mostrado no Teorema 2.4.1, mostrando que se existe uma diversidade mínima entre os classificadores, os dados rotulados por um classificador para serem aprendidos pelo outro permitiram que a diferença entre $d\left(h_{j}^{(i)}, h^{*}\right)$ diminua proporcionalmente ao número de iterações do algoritmo 
Cotraining.

Teorema 2.4.1 Dado um conjunto rotulado inicial $L$, e assumindo que o tamanho $l$ do mesmo é suficiente para treinar dois classificadores $h_{0}^{(1)}$ e $h_{0}^{(2)}$ dentro do contexto do aprendizado PAC (Valiant, 1984). Se $h_{0}^{(1)}$ seleciona u exemplos não rotulados para atualizar $L$ e posteriormente $h_{1}^{(2)}$ é treinado com $L$, e sendo $b_{1}$ e $\delta$ definidos pelo aprendizado PAC, então se cumpre que:

$$
P\left(d\left(h_{1}^{(2)}, h^{*}\right) \geq b_{1}\right) \leq \delta
$$

Os autores afirmam que o aprendizado multi-visão é um caso especial do aprendizado baseado em desacordo, e o fato de ter visões independentes é uma condição suficiente e não necessária para o sucesso do algoritmo. Em outras palavras, quando existem duas visões explícitas nos atributos dos dados, a diversidade entre os classificadores é atingida e, portanto, o desacordo entre eles permite o sucesso no processo de ajuda. Quando não existem duas visões dos dados, a diversidade entre os classificadores pode ser alcançada através de outras estratégias.

Com o objetivo de gerar bons classificadores, é interessante notar que o Aprendizado Baseado em Desacordo, quando o desacordo é provido por diferentes algoritmos, pode ser visto também como Aprendizado por Comitês de Classificadores (ensembles). Como já comentado, o aprendizado semi-supervisionado afirma que o uso de duas visões independentes dos dados não rotulados é suficiente para obter bons classificadores e, portanto, nesse caso, não existiria necessidade de combinar diferentes classificadores. Por outro lado, técnicas baseadas em comitês de classificadores afirmam que com a combinação de classificadores com desacordo suficiente entre as suas hipóteses conseguem obter bons resultados e, portanto, não seria necessário utilizar os dados não rotulados no processo de treinamento (Kuncheva e Whitaker, 2003). Nesse contexto, o Aprendizado Baseado em Desacordo , como proposto nesta dissertação, pode combinar tais técnicas para definir um modelo de aprendizado capaz de gerar comitês de classificadores robustos aproveitando a grande quantidade de dados não rotulados disponíveis para o treinamento dos mesmos (Zhou, 2009).

\subsection{Trabalhos Relacionados}

Tanto o algoritmo Cotraining como suas variações já foram utilizados para resolver diferentes problemas de aprendizado, incluindo classificação de textos (Blum e Mitchell, 1998; Rayid, 2001; Nigam e Ghani, 2000; Laguna e Lopes, 2009), reconhecimento de entidades nos textos (Collins e Singer, 1999), processamento de linguagem natural (Pierce e Cardie, 2001), classificação de imagens (Yan e Naphade, 2005), entre outras. Estas aplicações mostram a importância do algoritmo em diferentes áreas nas quais é possível gerar dois conjuntos de atributos independentes dos dados para formar as duas visões.

Na grande maioria de cenários, no entanto, existe uma visão dos dados ao invés de duas, pelo que a usabilidade do algoritmo Cotraining é limitada. Nesse contexto, a necessidade 
de suavizar o pressuposto de independência teve uma grande atenção na comunidade científica, como já mencionado. Os resultados mostram que suavizar esse pressuposto para utilizar visões não independentes ainda permite resultados bons. Nesse contexto, variações do Cotraining que não utilizam explicitamente duas visões dos conjuntos de atributos foram desenvolvidas. Estas variações do Cotraining posicionam-se dentro dos algoritmos de aprendizado semi-supervisionado mono-visão com múltiplos classificadores, que por sua vez é uma parte do aprendizado baseado em desacordo.

Nigam e Ghani (2000) fizeram experimentos para comparar o algoritmo Cotraining com os modelos do Expectation Maximitation (EM). Devido ao fato que nem todas as bases de dados apresentam duas partições de atributos independentes, geraram as duas partições aleatoriamente sobre os atributos da única visão. Os resultados empíricos sugerem que quando existe uma quantidade grande de atributos, gerar as duas visões através de duas partições aleatórias dos mesmos atinge resultados bons, até melhor do que outros algoritmos semi-supervisionados como são o EM e o Selftraining. No entanto, quando a quantidade de atributos não é tão elevada, esta abordagem não apresenta um desempenho aceitável. Zhang e Zheng (2009) estenderam a idéia de partição de atributos, escolhendo atributos ortogonais para cada visão. Para tal objetivo, os autores propuseram utilizar a técnica PCA (Principal Component Analysis) para projetar os dados no subespaço ortogonal e gerar subconjuntos de atributos menos correlacionados possível.

Goldman e Zhou (2000) utilizaram o algoritmo Cotraining para treinar dois classificadores com diferentes algoritmos de aprendizado e uma visão dos dados. Este método requer que cada classificador particione o espaço de exemplos em classes de equivalência (subconjuntos da mesma classe da mesma forma que fazem as àrvores de decisão). $\mathrm{O}$ método para combinar os classificadores foi rotular os exemplos quando os classificadores concordarem. Em caso dos classificadores discordarem, a acurácia de cada classificador sobre as classes de equivalência era calculada com 10-fold cross validation, de maneira que era possível compará-los e determinar o rótulo de novo exemplo. Embora esta abordagem conseguiu suavizar bastante o pressuposto de independência, é muito restritiva para escolher os classificadores, pois nem todos trabalham com o conceito de classes de equivalência. Zhou e Goldman (2004) estenderam o algoritmo para trabalhar com mais classificadores, rotulando um exemplo por votação ponderada pela confiança de classificação.

Li (2005) propôs a TriTraining, que faz uso de três classificadores baseados no mesmo algoritmo de aprendizado mas utilizando diferentes conjuntos de treino. Ela difere do modelo do algoritmo Cotraining pelo fato de não ser multi-visão necessariamente, pois a base do algoritmo TriTraining é o uso dos 3 classificadores. Se pelo menos dois dos classificadores coincidirem na classificação de um exemplo, o exemplo é rotulado e utilizado para atualizar o conjunto de treino. A idéia básica desta abordagem é que a maioria ensina à minoria. Li e Zhou (2007) propuseram o método Co-forest, que é uma extensão do algoritmo TriTraining para utilizar mais classificadores, utilizando a teoria de ensembles para a predição final do rótulo. 
Apesar de várias pesquisas terem sucesso na aplicação do algoritmo Cotraining em domínios nos quais os atributos são gerados a partir de uma mesma visão, a teoria clássica do aprendizado semi-supervisionado multi-visão não consegue explicar formalmente estes resultados. Wang e Zhou (2007) apresentaram resultados teóricos que mostram que o motivo do sucesso dos métodos que combinam diferentes classificadores é a diversidade de informação entre os algoritmos de aprendizado, não importando se essa diversidade é atingida utilizando mais de uma visão, diferentes algoritmos de aprendizado, diferente calibração de parâmetros nos algoritmos ou outros recursos. Kuncheva (2004) mostra mais detalhe da teoria tradicional para combinar diversos classificadores, e (Zhou e Li, 2009) mostra uma teoria mais detalhada sobre os métodos que combinaram classificadores, dentro do novo paradigma chamado Aprendizado Semi-supervisionado baseado em Desacordo. 


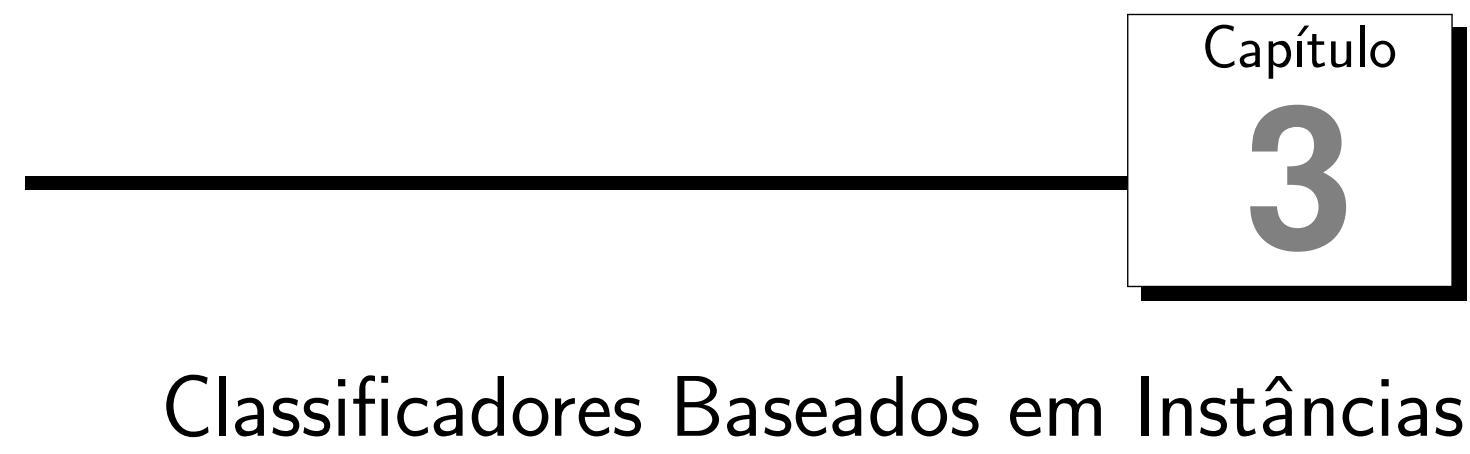

\subsection{Considerações Iniciais}

A hipótese induzida em uma grande quantidade de algoritmos de aprendizado é construída em uma etapa de treinamento dos classificadores. Esses algoritmos são conhecidos como gulosos ou eagers, pois a generalização ou indução da hipótese se dá nessa etapa prévia (Mitchell e Blum, 1997). Entretanto, existe um outro tipo de algoritmo que simplesmente armazena os exemplos na etapa de treino e todo o processamento para a predição do rótulo é feito na etapa de classificação. Por essa característica, esses algoritmos são conhecidos como baseados em instâncias ou lazy (preguiçosos) (Aha et al., 1991).

Uma das características principais dos algoritmos baseados em instâncias é que a predição do rótulo de um exemplo novo depende dos rótulos dos exemplos mais similares. Logicamente, o principal pressuposto desses algoritmos é que exemplos similares terão rótulos similares (Aha et al., 1991). Quando é necessário rotular algum exemplo novo, um subconjunto de exemplos similares são recuperados da memória e utilizados para predizer o rótulo daquele exemplo. Hastie et al. (2003) denominam este tipo de métodos como métodos livres de modelo, devido ao fato que ao invés de generalizar um modelo específico global para predizer os rótulos, calculam uma aproximação local diferente para cada exemplo novo a ser classificado. Estes métodos têm vantagem nos casos em que a função objetivo é muito complexa para ser generalizada completamente, mas é possível definí-la por uma coleção de funções de aproximações locais menos complexas (Mitchell e Blum, 1997). Embora não haja custo associado na etapa de treino desses classificadores, o custo da predição do rótulo de novos exemplos é alto devido à alta complexidade da tarefa de recuperação dos exemplos similares em grandes base de dados.

O algoritmo mais tradicional do aprendizado baseado em instâncias é o algoritmo $K$-Vizinhos Mais Próximos (KNN), introduzido por Cover e Hart (1967). O KNN assume 
que é possível representar todos os exemplos da base no espaço n-dimensional, sendo a similaridade calculada usando alguma medida de distância. A idéia básica é recuperar, do conjunto de treinamento, os $k$ vizinhos mais similares ao exemplo a rotular e predizer o seu rótulo com base nos rótulos dos $k$ vizinhos mais próximos.

Um outro método importante do aprendizado baseado em instâncias é Case-Based Reasoning, que utiliza experiências passadas para entender e resolver novos problemas (Watson, 1995). Uma grande diferença com o algoritmo KNN é que este método não representa os dados no espaço euclidiano e contém etapas de adaptação dos casos recuperados, bem como de armazenagem desses novos casos. Outros métodos relacionados com o aprendizado baseado em instâncias são as Redes Neurais Probabilísticas (Specht, 1990), Redes de Funções de Base Radial (Powell, 1987), entre outros.

Um fator comum entre todos esses métodos é a necessidade de definir como calcular a similaridade entre exemplos. Especificamente, este trabalho considera uma abordagem relacionada com o algoritmo KNN. Por esse motivo, algumas medidas comumente utilizadas pelo KNN para calcular a similaridade são definidas a seguir.

\subsection{Medidas de Similaridade}

Especificamente em aprendizado de máquina, a similaridade é definida com base em uma função que combina as similaridades de cada atributo dos exemplos. Tal similaridade nos atributos é definida com base em alguma medida de distância, sendo que exemplos mais similares têm uma distância menor. Em geral, estes atributos podem ser de 3 tipos: contínuos, discretos e categóricos. Formalmente, pretende-se calcular a similaridade entre dois conjuntos de atributos da forma $\overrightarrow{x_{1}}=\left(a_{11}, \ldots, a_{1 d}\right)$ e $\overrightarrow{x_{2}}=\left(a_{21}, \ldots, a_{2 d}\right)$, sendo que cada atributo $a_{i j}$ pode ser de um tipo diferente. A seguir, são definidos as medidas mais utilizadas para cada tipo de dado.

\subsubsection{Dados Numéricos}

Os dados numéricos são facilmente representáveis no espaço n-dimensional. Por esse motivo, a medida mais utilizada para calcular a distância entre dois exemplos é a distância euclidiana, representada pela Equação 3.1.

$$
d\left(\overrightarrow{x_{1}}, \overrightarrow{x_{2}}\right)=\sqrt{\sum_{r=1}^{n}\left(a_{1 r}-a_{2 r}\right)^{2}}
$$

Nesse sentido, a similaridade entre os dois exemplos define-se como uma função complementar da distância, sendo usualmente utilizada o oposto $\operatorname{sim}\left(\overrightarrow{x_{1}}, \overrightarrow{x_{2}}\right)=-d\left(\overrightarrow{x_{1}}, \overrightarrow{x_{2}}\right)$ ou a função inversa $\operatorname{sim}\left(\overrightarrow{x_{1}}, \overrightarrow{x_{2}}\right)=1 / d\left(\overrightarrow{x_{1}}, \overrightarrow{x_{2}}\right)$. A distância euclidiana é a mais utilizada no contexto de aprendizado de máquina, sendo um padrão na aplicação de diversos algoritmos, especialmente do KNN (Aha et al., 1991). 
Outra função de distância utilizada no contexto do aprendizado de máquina é a distância de Manhattan. Como mostrado na Equação 3.2, simplesmente considera a soma geral das diferenças em valor absoluto de cada atributo.

$$
d\left(\overrightarrow{x_{1}}, \overrightarrow{x_{2}}\right)=\sum_{r=1}^{n}\left|a_{1 r}-a_{2 r}\right|
$$

Tanto a distância euclidiana quanto a distância de Manhattan são casos particulares da distância de Minkowsky, definida pela Equação 3.3. A distância de Manhattan é obtida com o valor de $p=1$ e a distância euclidiana é obtida com o valor de $p=2$.

$$
d\left(\overrightarrow{x_{1}}, \overrightarrow{x_{2}}\right)=\left(\sum_{r=1}^{n}\left|a_{1 r}-a_{2 r}\right|^{p}\right)^{1 / p}
$$

Uma desvantagem das medidas apresentadas é que sofrem do chamado problema da dimensionalidade, porque consideram que todos os atributos influenciam a distância com o mesmo peso (Bishop, 1995). Nesse contexto, uma outra medida proposta foi a distância de Mahanalobis (Mahalanobis, 1936), que consegue transformar o espaço para oferecer uma noção de distância mais representativa da variança dos mesmos. As distribuições elípticas e normais são casos indicados para aplicar esta distância. Dados dois vetores e a matriz de covariancia $S$, a distância de Mahanalobis pode ser calculada como mostrado na equação 3.4. Para os casos nos quais a matriz de covariancia $S$ é uma matriz diagonal ${ }^{1}$, a fórmula pode ser reduzida à forma mostrada na Equação 3.5, a qual é uma distância euclidiana normalizada pela variância de cada atributo. Como ilustrado na Figura 3.1, dados um exemplo a ser rotulado e dois pontos rotulados, o exemplo pode estar mais próximo do exemplo da classe " $\times$ " quando a distância euclidiana é utilizada, porém, utilizando a distância de Mahanalobis a distribuição dos dados é considerada segundo a variância dos atributos, e com isso, o exemplo será rotulado como sendo da classe "•".

$$
\begin{gathered}
d\left(\overrightarrow{x_{1}}, \overrightarrow{x_{2}}\right)=\sqrt{\left(\overrightarrow{x_{1}}-\overrightarrow{x_{2}}\right)^{T} * S^{-1} *\left(\overrightarrow{x_{1}}-\overrightarrow{x_{2}}\right)} \\
d\left(\overrightarrow{x_{1}}, \overrightarrow{x_{2}}\right)=\sqrt{\sum_{r=1}^{n} \frac{\left(a_{1 r}-a_{2 r}\right)^{2}}{\sigma_{r}^{2}}}
\end{gathered}
$$

Além dessas medidas, outras medidas de distâncias foram definidas visando atacar o problema da dimensionalidade. Algumas distâncias importantes são apresentadas nos trabalhos de Wilson e Martinez (1997) e Santini e Jain (1999).

\subsubsection{Dados Categóricos}

Quando os dados não são contínuos, nem existe uma relação de diferença de ordem entre eles, não é possível calcular a distância por atributos. Especificamente, o cálculo de

\footnotetext{
${ }^{1}$ Uma matriz diagonal é uma matriz quadrada na qual os valores são 0, com exceção da diagonal principal, nas quais não necessariamente tem valor nulo.
} 


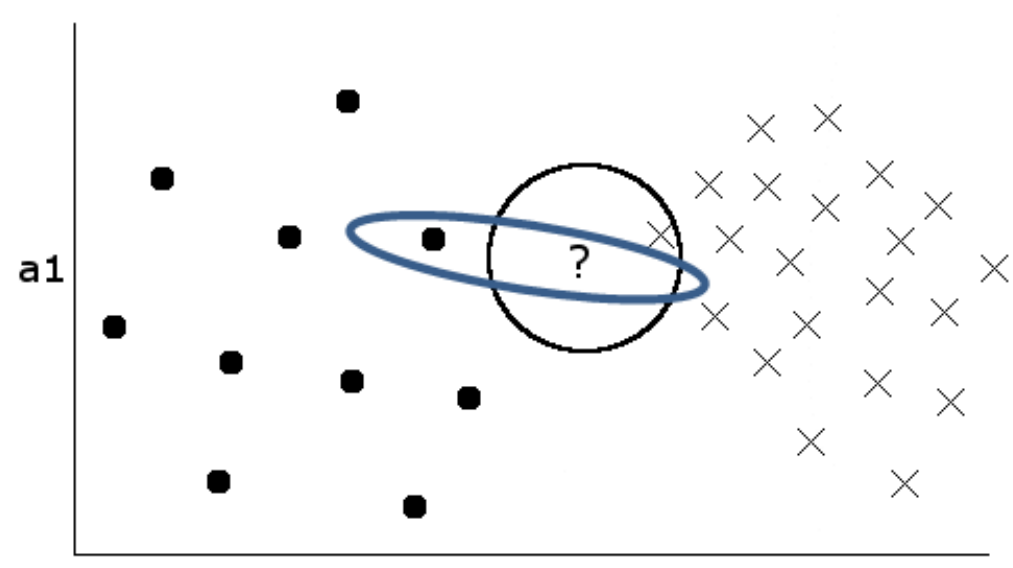

a2

Figura 3.1: Pela a distância euclidiana, o exemplo mais próximo do novo exemplo é da classe "×". Pela distância de Mahalanobis é "•".

$a_{1 r}-a_{2 r}$ não é viável por métodos para dados não numéricos devido a não existir uma definição de ordem inerente a este tipo de dados (Boriah et al., 2008). Nesse sentido, a maneira mais fácil, mostrada na Equação 3.6, é atribuir um valor de distância de 1 se são iguais, ou 0 se são diferentes.

$$
d_{\text {nom }}\left(a_{1 r}, a_{2 r}\right)= \begin{cases}1 & \text { se } a_{1 r}=a_{2 r} \\ 0 & \text { caso contrário. }\end{cases}
$$

Já no caso de um exemplo com dados tanto numéricos quanto categóricos é possível combinar as distâncias apresentadas anteriormente. Por exemplo, Kuncheva (2004) redefine a distância euclidiana com a Equação 3.7, sendo que a função $d_{a t}\left(a_{1}, a_{2}\right)$ retorna a distância entre os valores de dois atributos definida pela Equação 3.8.

$$
\begin{gathered}
d\left(\overrightarrow{x_{1}}, \overrightarrow{x_{2}}\right)=\sqrt{\sum_{r=1}^{n} d_{a t}\left(a_{1 r}, a_{2 r}\right)} \\
d_{a t}\left(a_{1 r}, a_{2 r}\right)= \begin{cases}\left(a_{1 r}-a_{2 r}\right)^{2}, & \text { se o atributo for numérico } \\
1-d_{\text {nom }}\left(a_{1 r}, a_{2 r}\right) & \text { se o atributo for categórico }\end{cases}
\end{gathered}
$$

Huang (1997) apresentou uma distância baseada na freqüência chamada medida de disimilaridade, para abordar o problema de agrupamento de dados com atributos nominais. Jiang et al. (2007) redefine essa distância como mostrado na Equação 3.9, sendo que $F\left(a_{i j}\right)$ representa a freqüência do valor do atributo $j$ de $x_{i}$ na base de dados.

$$
d\left(\overrightarrow{x_{1}}, \overrightarrow{x_{2}}\right)=\sum_{r=1}^{n} \frac{F\left(a_{1 r}\right)+F\left(a_{2 r}\right)}{F\left(a_{1 r}\right) * F\left(a_{2 r}\right)} * d_{a t}\left(a_{1 r}, a_{2 r}\right)
$$

Boriah et al. (2008) mostra outros tipos de distância para dados categóricos, e faz uma comparação experimental das diversas distâncias para resolver o problema de detecção de outliers ou ruído. 


\subsubsection{Outros Tipos de dados}

Embora os dados numéricos e categóricos sejam os mais utilizados para representar os dados em tarefas de mineração, existem outro tipo de representação específicas para certos domínios. Um dos domínios mais estudados é o textual, cujo estudo na mineração é conhecido como Mineração de Textos (Feldman e Sanger, 2006). A representação mais utilizada aplicada na mineração de textos com ênfase em métodos estatísticos leva a uma representação matricial do tipo tabela atributo-valor (Weiss et al., 2005). Nessa representação, denominada bag of words, cada documento é representado como um vetor de atributos (termos que ocorrem no texto) e valores (pesos que quantificam a presença ou freqüência de ocorrência dos termos nos documentos). Nessa abordagem a tabela atributo-valor pode eventualmente conter milhares de atributos representando os termos de toda a coleção de documentos.

Nota-se que a representação das freqüência é numérica, o que pode levar a pensar que a distância euclidiana é uma boa métrica para avaliar a similaridade. Porém, não é muito útil devido ao fato que textos de uma mesma classe podem ter diferentes frequiências de palavras, ocasionando distâncias com valores altos (e pouca similaridade) entre eles. Por exemplo, um texto completo e o seu resumo seriam considerados pouco similares. Uma das medida mais utilizada para calcular a similaridade entre textos é a medida do cosseno, a qual define a similaridade entre dois vetores como o cosseno do ângulo formado entre eles. Essa medida aproveita o fato do cosseno do ângulo formado por dois vetores ser igual ao produto escalar dos vetores dividido pelo produto dos módulos dos mesmos, como apresentado na Equação 3.10. Neste caso, o vetor $A$ representa o primeiro documento, e o vetor $B$ o segundo. Pode-se citar o trabalho de Goto et al. (2007) e Lee e Welsh (2005) como referências sobre diferente medidas de similaridade entre documentos no processo de classificação.

$$
\operatorname{sim}(A, B)=\frac{A \cdot B}{\|A\| *\|B\|}
$$

Uma outra representação de grande atenção na comunidade científica é a relacional. Nesta representação aproveitam-se as conexões explícitas que existem entre os dados para obter informação que possibilite o cálculo de similaridade (Getoor e Taskar, 2007). Tradicionalmente, estes tipos de dados são representados por grafos, sendo a similaridade de Jaccard (Jaccard, 1901) uma das mais utilizadas. Esta medida calcula a similaridade entre dois vértices de um grafo baseando-se na quantidade de vizinhos compartilhados. Seja $\Gamma_{i}$ a vizinhança do vértice $i$ e $\Gamma_{j}$ a do vértice $j$, a similaridade de Jaccard é calculada como mostrado na Equação 3.11. Leicht et al. (2006) apresentam diversas medidas de similaridade utilizadas no domínio relacional.

$$
\operatorname{sim}\left(v_{i}, v_{j}\right)=\frac{\left|\Gamma_{i} \cap \Gamma_{j}\right|}{\left|\Gamma_{i} \cup \Gamma_{j}\right|}
$$

Os métodos baseados em instâncias utilizam-se de definições de similaridade para 
aprender. Como já mencionado, o algoritmo KNN é um dois mais tradicionais e importantes algoritmos deste paradigma. Por esse motivo, o KNN é estudado com mais detalhe a seguir.

\subsection{Algoritmo K Vizinhos Mais Próximos}

O KNN é um algoritmo tradicional que apesar da sua simplicidade, mostrou-se robusto em tarefas de classificação em diversos domínios, sendo utilizado desde problemas de reconhecimento de dígitos até aprendizado de padrões elétricos do coração. Nesta seção uma análise do algoritmo é apresentada, assim como diversas variações propostas na literatura.

\subsubsection{Definição do Algoritmo}

Como já mencionado, o algoritmo KNN utiliza o conceito de distância para decidir quais exemplos pertencem a vizinhança do exemplo a ser rotulado. Por tal motivo, a distância euclidiana é a mais utilizada para o cálculo da similaridade entre os exemplos, porém, o algoritmo é independente da definição de distância utilizada. Com isso, dado um exemplo $x_{\text {novo }}$ que deve ser rotulado, obtêm-se os $k$ vizinhos mais similares com $x_{\text {novo }}$, representados por $k_{-} V i z i n h o s\left(x_{n o v o}\right)=\left\{\left(x_{1}, y_{1}\right) \ldots\left(x_{k}, y_{k}\right)\right\}$. A versão mais simples do algoritmo indica que o rótulo de $x_{i}$ é calculado com base na votação majoritária dos rótulos $y_{i}$ de cada exemplo do conjunto $k_{-}$Vizinhos. Como mostrado na Equação 3.12, faz-se uma contagem para cada classe do conjunto total de classes $C$. Os empates são resolvidos aleatoriamente, sendo que para o caso binário recomenda-se sempre utilizar valores de $k$ ímpares para evitar os empates.

$$
f\left(x_{\text {novo }}\right)=\underset{c \in C}{\operatorname{argmax}} \sum_{j=1}^{k}\left(c=y_{j}\right)
$$

Como pode ser notado, a classe predita pelo algoritmo depende de $k$, pois a distribuição dos rótulos da vizinhança pode variar, especialmente nas fronteiras entre os grupos formados por cada classe. Este comportamento pode ser observado na Figura 3.2, que ilustra uma situação na qual para diferentes valores de $k$ a classe majoritária muda. Por exemplo, para $k=1$ e $k=5$ a classe majoritária é "•" e para $k=3$ a classe majoritária é "×".

De uma forma mais geral, os limites de decisão do algoritmo podem mudar com o valor de $k$, sendo que quanto menor o valor de $k$, a tendência é formar subconjuntos de decisão separados. Entretanto, quanto maior o valor de $k$, os limites de decisão tornam-se mais uniformes e suaves, com tendência a gerar tantos subgrupos quantas classes existem. Esta característica do KNN pode ser observada na Figura $3.3^{2}$. Note-se que para $k=1$, a superfície de decisão é mais estrita, gerando diversos subgrupos isolados. Uma

\footnotetext{
${ }^{2}$ Imagens geradas com LocBoost Demo Applet. http://www.cs.technion.ac.il/ rani/LocBoost/
} 


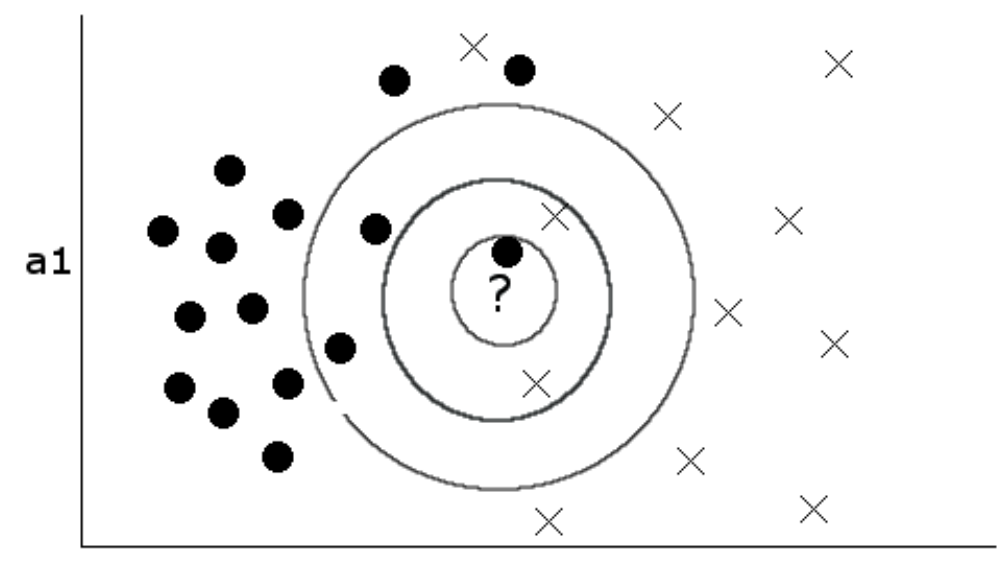

a2

Figura 3.2: Diferentes conjuntos de vizinhos para valores de $k=1,3$ e 5 .

característica importante é que o erro no conjunto de treinamento para $k=1$ é 0 , ou seja, exemplo nenhum está contido em regiões da outra classe. Já para $k=5$ a superfície de decisão mostra-se mais uniforme, não importando se alguns exemplos do conjunto de treinamento são incorretamente considerados como pertencentes a outra classe. Este tipo de dados são considerados outliers ou ruído nas classes. Em geral, quanto maior o valor de $k$, maior a tolerância ao ruído nas classes (Bishop, 1995), sempre que $k \ll N$, sendo $N$ o tamanho do conjunto de treinamento (Okamoto e Yugami, 2003). Ressalta-se que as superfícies de decisão formadas na figuras anteriores são complexas dificultando a sua representação mediante uma única função.

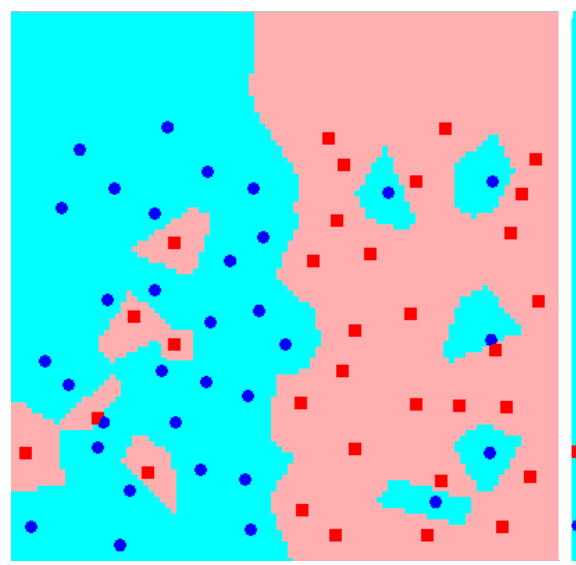

(a) $\mathrm{k}=1$

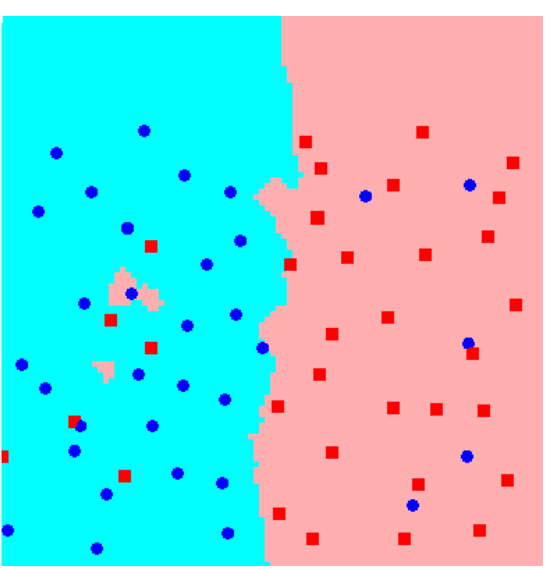

(b) $\mathrm{k}=5$

Figura 3.3: Limites de decisão para diferentes valores de $k$

Uma extensão natural do algoritmo para diminuir o impacto do ruído de classe é refinar o cálculo do rótulo do exemplo novo, atribuindo um peso à contribuição de cada vizinho proporcional com a similaridade (Dudani, 1976). Ou seja, aqueles que são mais similares têm uma contribuição maior do que aqueles vizinhos mais distantes. Este cálculo é definido segundo a Equação 3.13, sendo que $w_{i}$ é definida como o inverso do quadrado da distância entre as duas instâncias, como mostrado na Equação 3.14. 


$$
\begin{gathered}
f\left(x_{\text {novo }}\right)=\underset{c \in C}{\operatorname{argmax}} \sum_{j=1}^{k} w_{j} * I\left(c, y_{j}\right) \\
w_{j} \equiv \frac{1}{d\left(\text { xnovo }, x_{j}\right)^{2}}
\end{gathered}
$$

Independentemente da estratégia de predição do rótulo e da distância utilizada, o algoritmo KNN tem uma estrutura geral como mostrado no Algoritmo 3, sendo que todo o processo de inferência do algoritmo é feito na etapa de classificação.

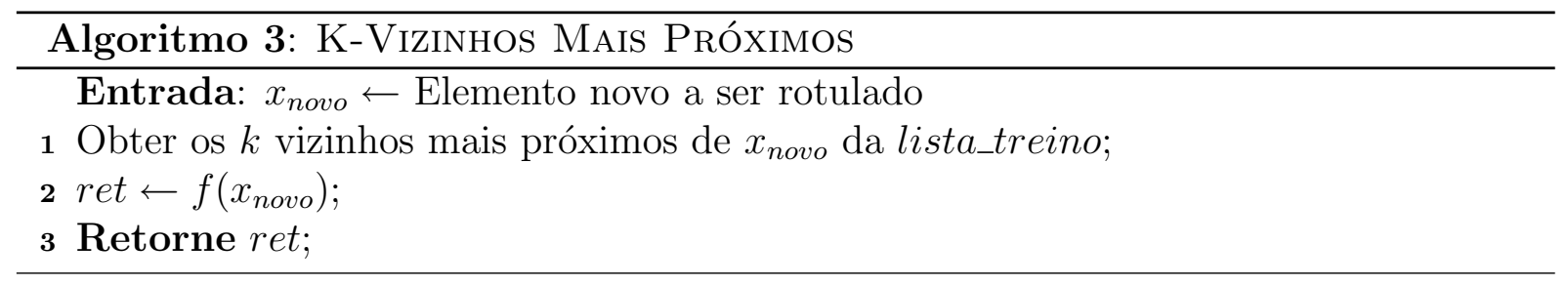

\subsubsection{Variações do Algoritmo}

O algoritmo KNN tem sido muito utilizado em diversos domínios. Com isso, algumas desvantagens do algoritmo em determinados domínios foram detetadas, sendo propostas algumas variações.

A primeira desvantagem que apresenta o algoritmo KNN é a sua complexidade alta na etapa de classificação de um novo elemento. Considerando uma estratégia de força bruta, para cada exemplo a ser rotulado é necessário percorrer o conjunto de treino completo para recuperar os $k$ vizinhos mais próximos e depois ordenar as distâncias. Considerando que $l$ exemplos rotulados estão disponíveis, a complexidade de obter os $k$ vizinhos mais próximos é $O(l+l * \log l) \approx O(l * \log l)$. Para rotular $u$ exemplos, a complexidade total da etapa de classificação é $O(u * l * \log l)$. Esta complexidade é muito alta, e até pode ser considerada inviável em alguns domínios. Alguns métodos de particionamento do espaço foram desenvolvidos com o objetivo de diminuir a complexidade da recuperação dos exemplos similares, como são KD-Tree (Bentley, 1975) e Projections Search (Papadimitriou e Bentley, 1980). Estes métodos utilizam estruturas complexas, que particionam o espaço em subgrupos para reduzir a complexidade de recuperação dos $k$ vizinhos mais próximos a $O(k * \log l)$. Porém, adicionam a complexidade de construção das estruturas de dados à etapa de treino. Por exemplo, na kd-tree a complexidade de construção da árvore binária é $O\left(l * \log ^{2} l\right)$. Além disso, adicionam complexidade de memória, o que pode ocasionar problemas de armazenamento de dados em bases muito grandes.

Frente ao problema de complexidade computacional (tanto em tempo de execução quanto em memória requerida), técnicas de redução do conjunto de treino foram propostas. Umas abordagens visam selecionar um subconjunto de treino de seleção, sendo chamadas de edição do KNN (edited KNN) (Wilson, 1972). Entre os principais trabalhos de edição do KNN pode-se citar a Gates (1972); Ritter et al. (1975); Aha et al. (1991), sendo que 
o seu estudo ainda continua tendo vigência como mostrado nos trabalhos de Jiang e hua Zhou (2004); Gil-Pita e Yao (2007); Fayed e Atiya (2009) entre outros.

Uma outra abordagem visa selecionar vértices que representem os agrupamentos, mesmo se tais vértices não existem no conjunto original. Esta abordagem é chamada de condensação de protótipos (Chang, 1974). Enquanto a edição do KNN está mais ligado com a idéia original do KNN, a condensação está mais ligada com uma classe de redes neurais conhecidos como Learning Vector Quantization (Kohonen et al., 2001). Estas redes utilizam um conjunto de protótipos, encontrados através da construção de redes neurais, para representar as classes. Quando um novo exemplo não rotulado estiver disponível o rótulo do protótipo mais próximo é atribuído. A condensação de protótipos utiliza tanto estratégias do aprendizado não supervisionado para encontrar os protótipos mais representativos, quanto estratégias do aprendizados supervisionado para rotular os novos exemplos. Entre os principais trabalhos pode-se citar a Domingos (1995), que define um subconjunto de regras de decisão que apresentam maior cobertura sobre os dados para classificá-los, e Decaestecker (1997) que além de encontrar os protótipos mais representativos utilizando o algoritmo K-Means (Lloyd, 1982), reduz a quantidade de protótipos utilizando a teoria de gradiente descendente para minimizar o erro na classificação.

O objetivo comum das duas abordagens é diminuir a quantidade de memória necessária para salvar os dados, agilizar o processo de classificação de novos dados, desconsiderar o ruído no conjunto de treino, e melhorar o desempenho na classificação. Para maiores detalhes sobre as técnicas de redução, ver o trabalho de Wilson e Martinez (2000).

Uma outra desvantagem mais ligada com o tipo de distância utilizado é a grande intolerância ao atributos irrelevantes. Tipicamente, a medida de distância é calculada utilizando todos os atributos, não importando se alguns atributos são menos informativos do que outros. Por exemplo, a distância euclidiana assume que todos os atributos contribuem com o mesmo peso no cálculo da distância. Como já mencionado, esta desvantagem é conhecida como de problema da dimensionalidade, e o algoritmo KNN é especialmente sensível a este problema (Mitchell e Blum, 1997).

Uma solução drástica para o problema da dimensionalidade é selecionar aqueles atributos mais informativos e descartar os menos relevantes (Liu e Motoda, 1998). Porém, é possível perder qualidade na classificação reduzindo consideravelmente a dimensão dos atributos. Nesse sentido, Han (1999) propôs colocar um peso em cada atributo para refinar o sua contribuição no cálculo da distância, sendo que os atributos mais relevantes tem um peso maior. Por exemplo, tomando como base a distância euclidiana, poderia redefinir-se a distância entre dois exemplos como mostrado na Equação 3.15.

$$
d\left(\overrightarrow{x_{1}}, \overrightarrow{x_{2}}\right)=\sqrt{\sum_{r=1}^{n} w_{r} * d_{a t}\left(a_{1 r}, a_{2 r}\right)}
$$

Uma terceira desvantagem é o fato do que o desempenho do KNN é altamente sensível ao valor de $k$. Embora com valores maiores de $k$ os limites de decisão vão se uniformizando, 
valores ditintos de $k$ implicam diferentes comportamentos do algoritmo, em especial na fronteira de decisão. Portanto, uma idéia inicial é testar todos os valores de $k$ e escolher o melhor dentro de um domínio. Zhipeng et al. (2002) propuseram testar diferentes valores de $k$ para cada novo elemento a ser rotulado, escolhendo aquele que tenha melhor desempenho quando gerado um classificador nä̈ve bayes a partir da vizinhança. Nesta proposta são apresentados resultados interessantes, porém é muito mais custosa do que o KNN normal, limitando a sua aplicação. Jiang et al. (2007) apresentam um levantamento bibliográfico interessante sobre as desvantagens do KNN e as modificações propostas na literatura, bem como propuseram escolher o melhor valor de $k$ utilizando cross-validation no conjunto de treino.

\subsubsection{Grafos baseados em Similaridade}

No Capítulo 2 foram introduzidos os conceitos do aprendizado baseado em grafos. A idéia geral desses métodos é gerar grafos nos quais as arestas representem a similaridade entre os exemplos. Os métodos baseados em grafos são bastante utilizados no aprendizado não supervisionado (Schaeffer, 2007), sendo conhecidos como Graph Clustering. Nesses métodos, é usual manter um número máximo de vizinhos aos quais um vértice pode ser conectado, definido por uma variável $k$. Por esse motivo, esses grafos são chamados grafos ou redes KNN, existindo vários trabalhos no contexto de aprendizado não supervisionado. Por exemplo, Karypis et al. (1999) propõe gerar uma rede KNN, e particioná-la em pequenos grupos que representem componentes altamente conectados. Após esse processo, juntam-se os grupos procurando maximizar os conexões dentro dos grupos e minimizar as conexões de similaridade com outros grupos.

As técnicas de agrupamento baseadas em grafos foram estendidas para servir de classificação, especialmente classificação semi-supervisionada. Uma vez que os grupos são obtidos, atribui-se a cada grupo um rótulo dependendo em algum critério (Zhu, 2005). Nesse sentido, as redes KNN tiveram uma atenção importante, pois permitem aproveitar na classificação semi-supervisionada uma característica interessante que é sua capacidade de modelar formas e curvas na distribuição dos dados que outros métodos não conseguem (Schaeffer, 2007). Esta característica esta baseada no manifold assumption, introduzido no Capítulo 2. Um exemplo é mostrado na Figura 3.4, sendo que os dados encontram-se em uma hiper-curva que dificilmente outros métodos poderiam modelar.

O processo de formação das redes KNN é incremental, no qual cada vértice é conectado com os seus $k$ vizinhos mais próximos. Existem duas abordagens principais para a formação das redes KNN, as simétricas e as mútuas. As redes KNN simétricas colocam uma aresta para cada vizinho mais próximo de cada vértice, sendo que um vértice poderia ter mais de $k$ arestas. As redes KNN simétricas foram inicialmente utilizadas na área de processamento de imagens (Harwood et al., 1987), sendo aplicadas posteriormente na área de aprendizado de máquina, especialmente no aprendizado não supervisionado (Schaeffer, 2007; Hutter et al., 2007). Entretanto, as redes KNN mútuas colocam uma aresta entre 


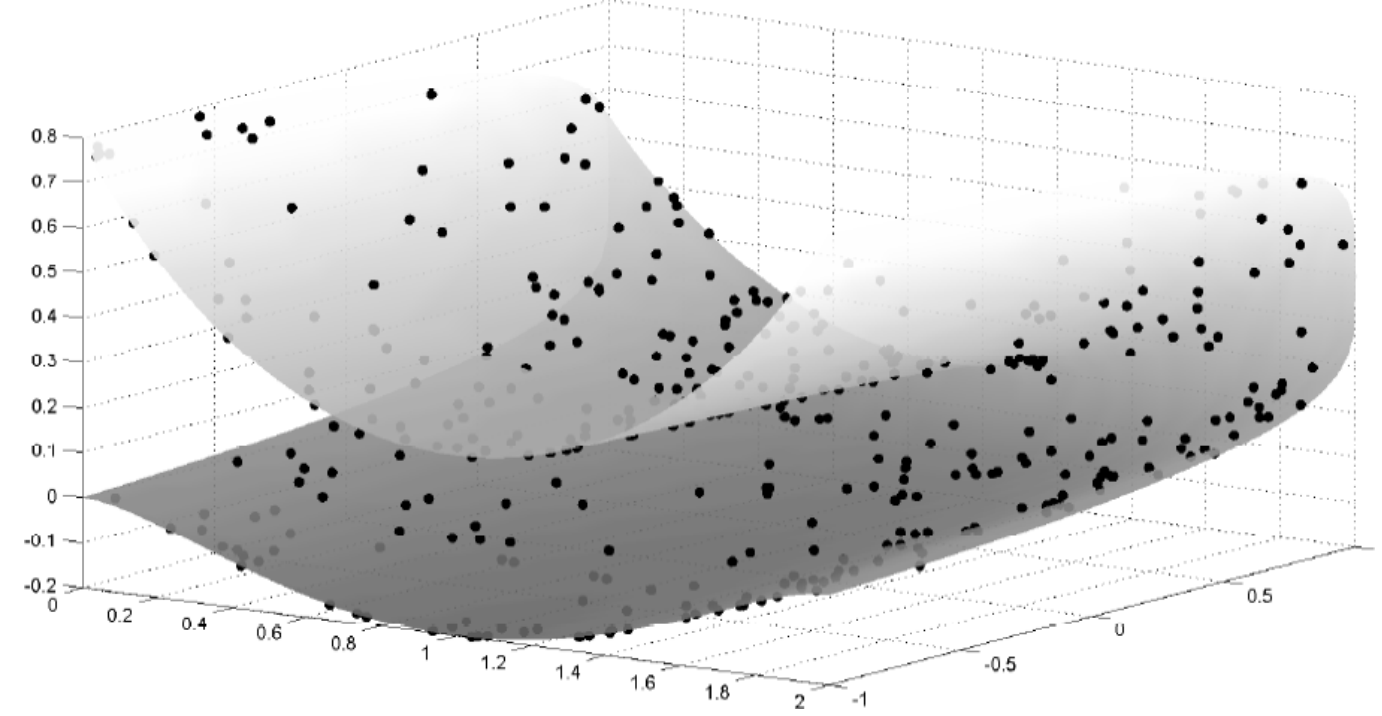

(a)

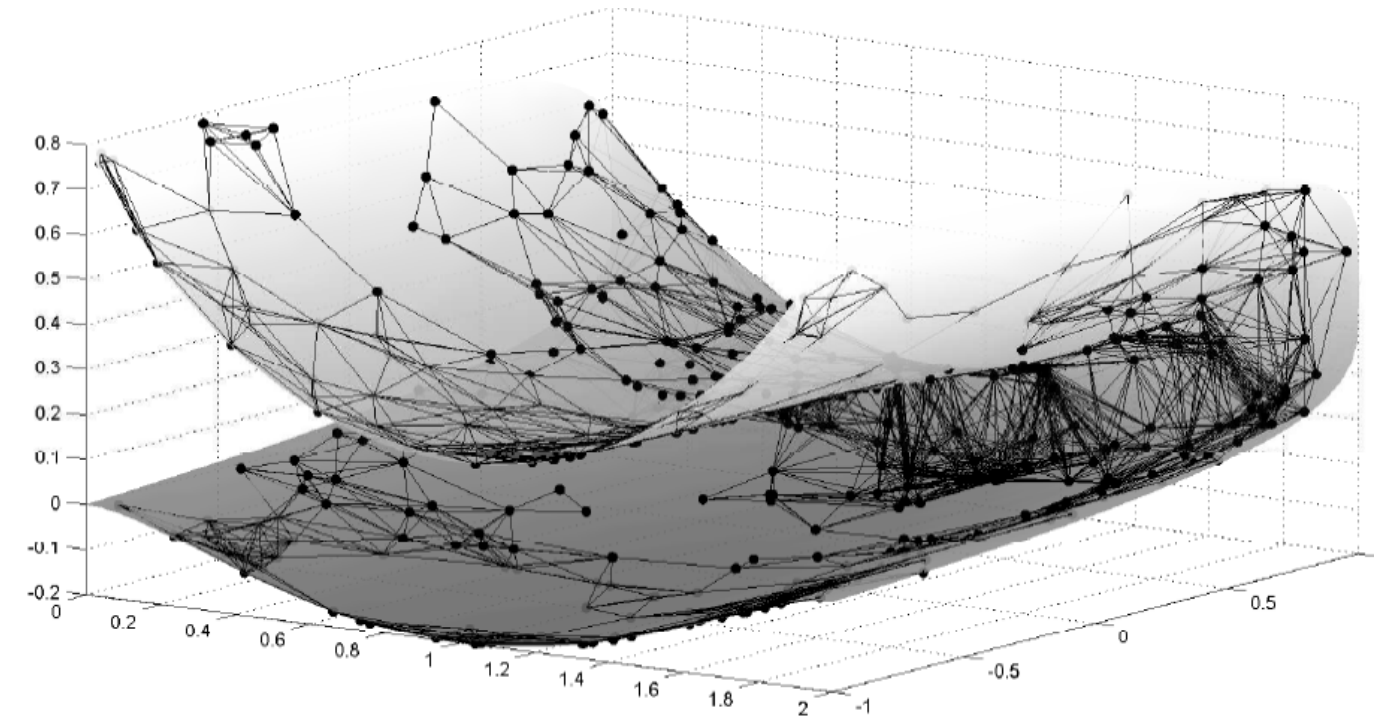

(b)

Figura 3.4: Exemplo de um modelo de similaridade baseado em grafos, sendo 3.4a a distribuição dos dados e 3.4b o modelo alcançado pelas redes KNN. Imagem modificada de Hein e Luxburg (2007)

dois vértices somente se a regra de vizinhança se cumpre mutuamente, ou seja, existe uma aresta entre $x_{i}$ e $x_{j}$, se e somente se $x_{i} \in k_{-} \operatorname{Vizinhos}\left(x_{j}\right) \wedge x_{j} \in k_{-} \operatorname{Vizinhos}\left(x_{i}\right)$. Por esse motivo as redes KNN mútuas são consideradas mais restritivas, sendo utilizadas tradicionalmente no aprendizado não supervisionado (Gowda e Krishna, 1978; Brito et al., 1997). Contudo, os processos atuais de geração de redes KNN utilizam a nomenclatura redes KNN para se referir as redes KNN simétricas, pois são as mais utilizadas no processo de geração de grafos de similaridade (Schaeffer, 2007).

Na Figura 3.5 é ilustrado o processo de formação das redes KNN simétricas. Notase que para este exemplo, dois componentes separados foram obtidos, mas nem sempre isto acontece. Por esse motivo, existem diversas pesquisas que visam dividir os grafos em possíveis grupos que definam as classes (Blum e Chawla, 2001; Zhu, 2005). Para um maior 
detalhe matemático-geométrico da formação das redes KNN, ver Eppstein et al. (1997).

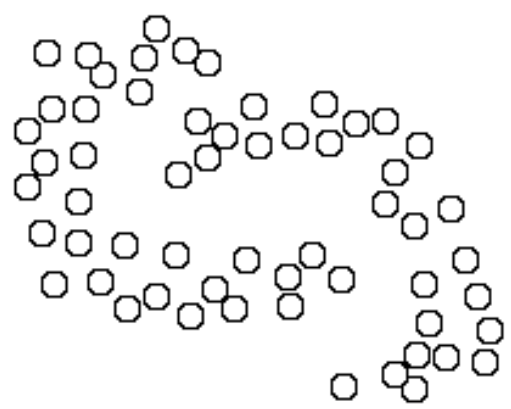

(a)

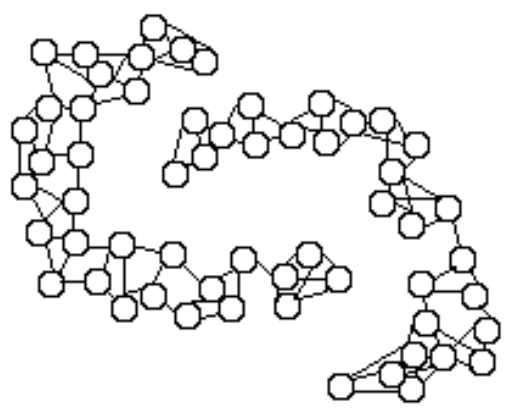

(c)

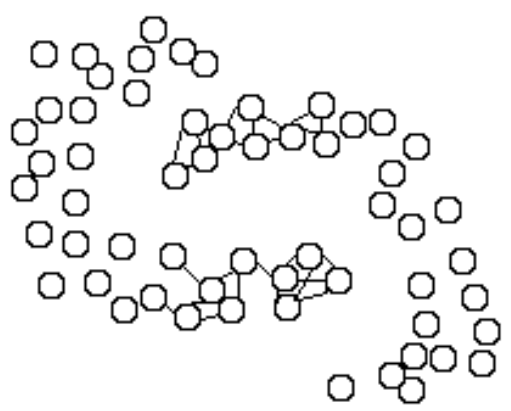

(b)

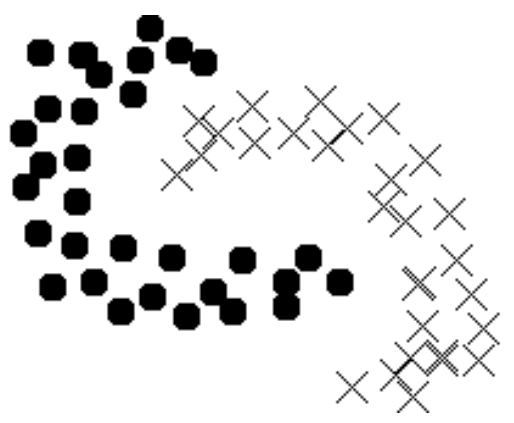

(d)

Figura 3.5: Exemplo de formação da rede KNN simétrica para $k=3$ onde 3.5a apresenta a distribuição de dados inicial, 3.5b e 3.5c o processo de formação do grafo e $3.5 \mathrm{~d}$ a distribuição real das classes

Também é possível aplicar técnicas do aprendizado relacional aos grafos KNN. Diferentes técnicas de classificação foram propostas no cenário relacional como a Inferência Coletiva, introduzida no Capítulo 2 ou a Classificação Baseada em Links (Getoor e Diehl, 2005), a qual utiliza os padrões similares entre os links dos vértices para rotular novos vértices. Recentemente, a teoria das Redes Complexas (Newman, 2003) tem ganhado uma grande atenção da comunidade científica dentro do domínio relacional, devido ao fato que provê um arcabouço teórico que permite caracterizar redes com uma enorme quantidade de vértices e arestas a partir de medidas especiais para tais objetivos. Por exemplo, as medidas de detecção de comunidades e recuperação de vértices importantes são medidas muito utilizadas das Redes Complexas. Nesse contexto, alguns trabalhos já aplicaram as estratégias das técnicas relacionais, os modelos e as medidas das Redes Complexas para estudar as redes de similaridade com muito bons resultados (Motta et al., 2008; Lathia et al., 2008; Motta e Lopes, 2009).

\subsection{Trabalhos Relacionados}

Apesar de ser um algoritmo tradicional, existem diversas aplicações e pesquisas recentes que estudam as variações e propriedades do KNN (Zhang et al., 2006; Schaeffer, 2007; Song et al., 2007; Wang et al., 2008; Fayed e Atiya, 2009; Motta e Lopes, 2009), entre outras. A proposta desta dissertação está relacionada com uma nova forma de definir 
a vizinhança de um exemplo, independente da medida de distância utilizada. Nesse contexto, alguns trabalhos já exploraram diferentes opções.

Song et al. (2007), por exemplo, definem o conceito de informatividade para escolher dentro dos $k$ vizinhos os mais informativos, sendo esta abordagem chamada de IKNN. São considerados vértices informativos aqueles que ficam mais próximos de vértices da mesma classe e mais longe de vértices de outras classes. Para o exemplo mostrado na Figura 3.6, a medida de informatividade consegue predizer o rótulo corretamente, embora os 7 vizinhos mais próximos indiquem o contrário.

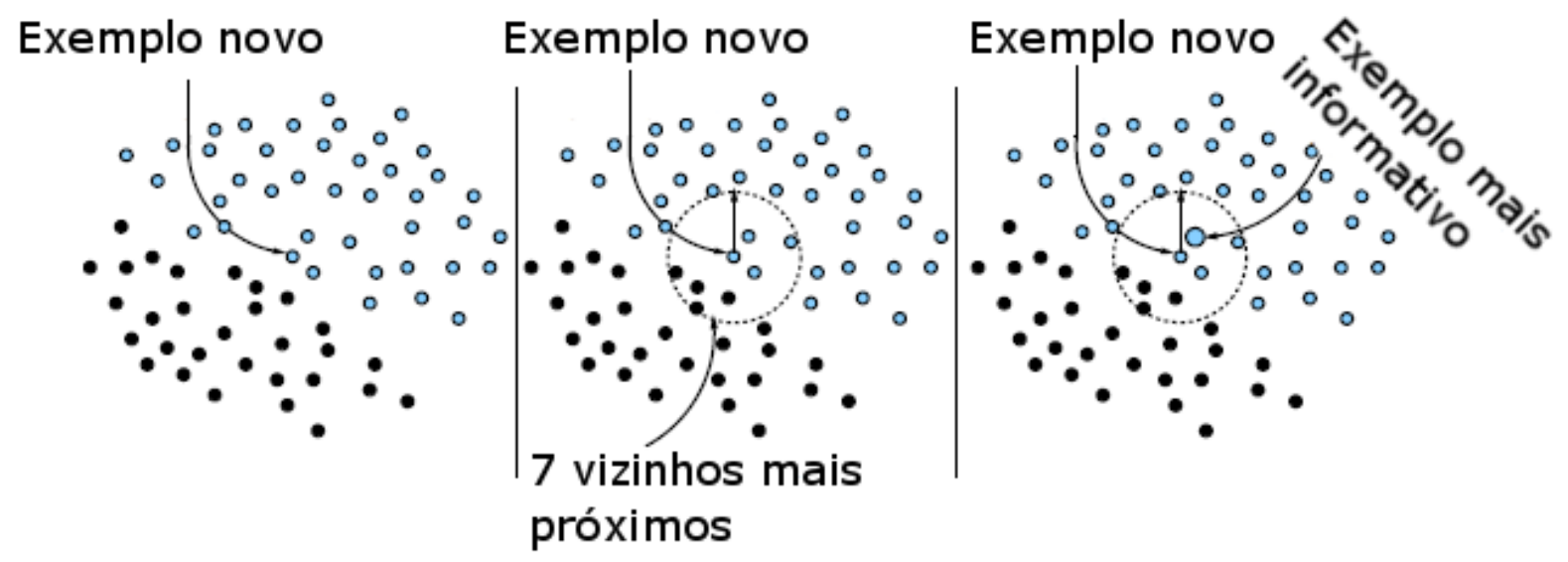

Figura 3.6: Vértice mais informativo dentre dos $k$ vizinhos mais próximos do novo exemplo

Weinberger e Saul (2009) induziram um classificador KNN com a distância de Mahalanobis, sendo esta estratégia chamada de LMNN (Large Margin Nearest Neighbor). Esta estratégia escolhe um subconjunto de vértices objetivando encontrar fronteiras que separam os exemplos de diferentes classes, enquanto visa diminuir a distância entre exemplos da mesma classe. Os resultados mostram que o uso da distância de Mahanalobis permite adicionar ao cálculo da distância componentes estatísticos que a distância euclidiana não considera, alcançando classificadores com melhor desempenho do que as SVM.

A idéia da regra do KNN simétrico (SKNN), aplicado em grafos, também foi explorado dentro do contexto de classificação. Nock et al. (2000) propuseram atualizar o conjunto de vizinhos de um exemplo com aqueles vértices que contenahm o exemplo novo dentro dos $k$ vizinhos mais próximos. Na figura 3.7 é ilustrado como é possível que os vizinhos mais próximos de um exemplo sejam incrementados dependendo da distribuição dos dados. Nota-se que enquanto $x 1$ consegue incrementar o conjunto de vizinhos, $x 2$ mantém o conjunto. Devido a alta complexidade para o cálculo dos vértices adicionais, os autores 
propõem a utilização de árvores para encontrar os vizinhos de todas as folhas de forma mais eficiente. Em um trabalho posterior, Nock et al. (2003) aplicam o SKNN no domínio relacionado com a normalização da poluição tropical, apresentado resultados interessantes com desempenho melhor do que o KNN. O trabalho proposto nesta dissertação visa encontrar uma extensão da vizinhança com uma estratégia similar com a SKNN, sendo que ao invés de incrementar a vizinhança de um exemplo, é proposto tratar cada conjunto como uma visão diferente de vizinhança em uma abordagem semi-supervisionada no estilo Cotraining.

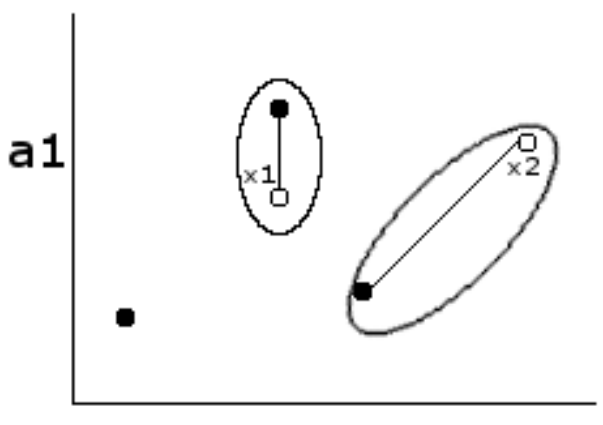

a2

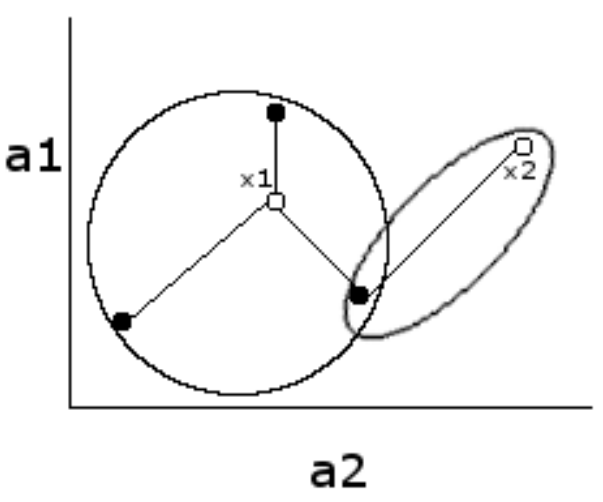

(b) $1-\mathrm{SNN}$

Figura 3.7: Diferentes conjuntos de vizinhos encontrados pelas estratégias KNN e SKNN para $k=1$. Imagem modificada de Nock et al. (2000)

Motta e Lopes (2009) propuseram a aplicação dos conceitos das Redes Complexas (Newman, 2003) para modelar as redes de similaridade entre objetos e induzir classificadores relacionais que explorem as estruturas de tais redes. Os autores propõem uma forma nova de criar as redes de similaridade chamada de Redes K-Associados. Nestas redes, diferentes componentes são gerados em um processo de construção da rede similar com a construção da rede KNN, com a diferença que somente exemplos da mesma classe possuem conexões. Com a rede gerada, é possível calcular a pureza de cada componente, sendo essa medida utilizada como uma probabilidade apriori dos vértices de um componente pertencer à classe do componente. Quando é necessário rotular um novo exemplo, os autores propõem combinar duas visões chamadas de Teste-Rede e Rede-Teste. A visão Teste-Rede é simplesmente a obtenção dos $k$ vizinhos mais próximos na rede, e a visão Rede-Teste é formada pelos vértices da rede que contém o vértice novo dentro dos seus $k$ vizinhos mais próximos. A Figura 3.8 ilustra as duas visões para um mesmo exemplo. Dentro das estratégias propostas de classificação, definiu-se dois classificadores que combinam essas duas visões com um comitê de classificadores. O primeiro classificador rotula o exemplo somente se as duas visões concordarem, o segundo classificador atribui a probabilidade máxima dentre as duas visões. Como resultados deste trabalho obtevese que o primeiro classificador apresenta uma precisão alta, porém, a cobertura diminui substancialmente porque não se garante a classificação de todos os dados. O segundo clas- 
sificador mostrou-se mais robusto e com melhor desempenho nos casos em que a escolha do valor de $k$ é ótima. Nesse trabalho o autor investiga a combinação das duas visões para o domínio relacional dentro de uma abordagem supervisionada. Por outro lado, neste trabalho propõe-se a combinação dessas duas visões para qualquer domínio em uma abordagem semi-supervisionada, gerando classificadores diferentes separadamente com cada visão. Esta abordagem é detalhada no Capítulo 4.
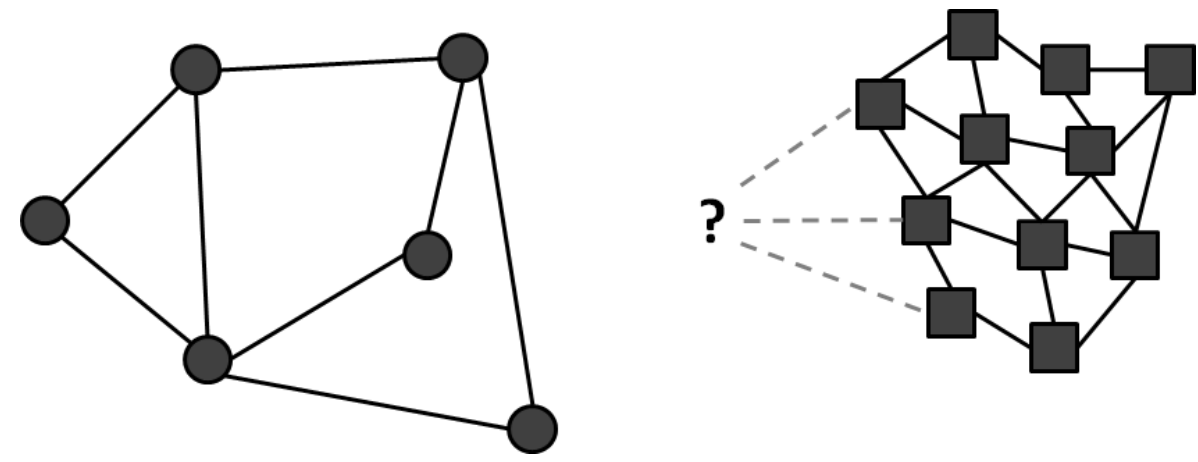

(a) teste-rede
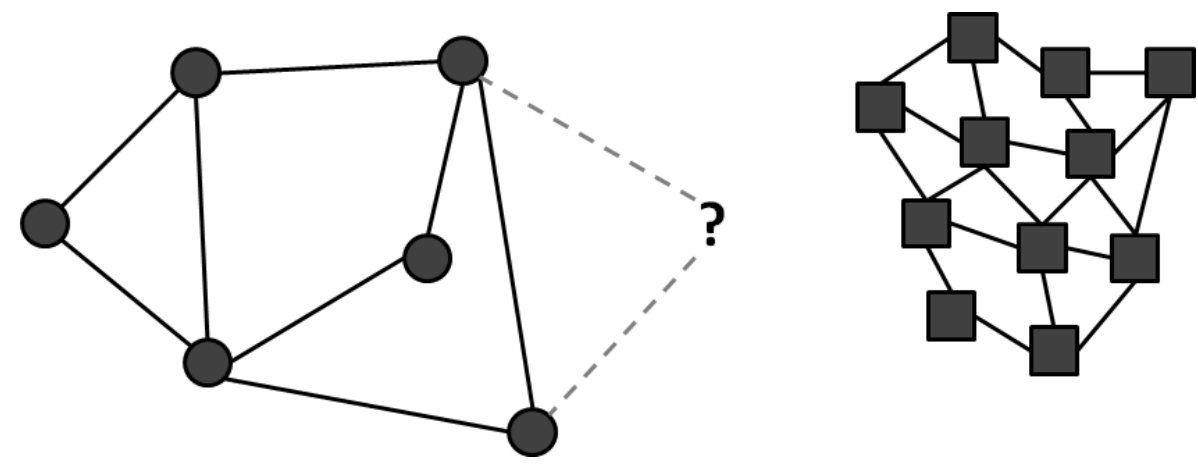

(b) rede-teste

Figura 3.8: Exemplo de obtenção da vizinhança na rede k-associados com a visão testerede e rede-teste para uma rede com $k=3$. Figura modificada de Motta e Lopes (2009)

Conforme o aprendizado semi-supervisionado foi ganhando maior atenção pela comunidade científica, diversas extensões do KNN foram propostas. Como afirma Xiong et al. (2006), existem duas extensões naturais do KNN para o aprendizado semi-supervisionado. O primeiro é o KNNS ( $k$ nearest neighbor based semi-supervised), também conhecido como propagação de rótulo, no qual cada exemplo rotulado vai propagando o seu rótulo entre os seus $k$ vizinhos (somente propaga para os exemplos não rotulados). Em caso de empate, diversas estratégias podem ponderar o resultado final. Uma outra abordagem natural é o TOP-KNNS (top- $k$ nearest neighbor), o qual age da mesma forma que a abordagem supervisionada para rotular um novo exemplo, mas atualiza o conjunto de treino incrementalmente a medida que mais exemplos são rotulados. Esta abordagem pode-se entender como uma estratégia Selftraining na qual em cada iteração rotula-se somente um exemplo.

Guan et al. (2008) propõem uma abordagem semi-supervisionada para editar o conjunto de dados rotulados. A abordagem proposta pelos autores adiciona um processo inicial de pseudo-classificação no qual os vértices não rotulados são classificados para escolher dentre os dados rotulados o melhor subconjunto que representa da melhor forma 
a distribuição dos dados com o objetivo de melhorar o desempenho do classificador KNN final. Esse processo de pseudo-classificação prévio é feito com Cotraining, dividindo os atributos em 3 visões não independentes como Ensembles. Os autores denominam esta abordagem de En-Cotraining e a comparam com os métodos tradicionais de edição do conjunto de vizinhos, obtendo melhores resultados. Ressalta-se que, neste caso, o aprendizado semi-supervisionado é utilizado exclusivamente para a edição do conjunto de exemplos rotulados, sendo essa uma estratégia custosa em termos computacionais.

A proposta deste trabalho, segundo as nossas pesquisas, encontra-se mais alinhada com a proposta de Zhou e Li (2005). Nessa proposta, os autores modificam o algoritmo Cotraining para ser aplicado na tarefa de regressão. O algoritmo proposto é chamado de COREG, o mesmo que utiliza dois regressores KNN que se ajudam mutuamente no processo de predição dos dados contínuos. Os autores fundamentam o uso de regressores KNN devido a sua natureza preguiçosa para o treino. Como o algoritmo Cotraining é um processo de treino contínuo, a utilização de outro tipo de regressores teria um custo computacional maior. Os dois regressores não pressupõem que existam duas visões independentes dos dados, sendo a diferença entre os regressores obtida pela medida de distância utilizada por cada regressor. As duas medidas estão baseadas na distância de Minkowsky, utilizando diferente parâmetro $p$. O sucesso desse trabalho fundamenta-se no paradigma de Aprendizado Semi-supervisionado baseado em Desacordo, introduzido no Capítulo 2. Evidentemente, a regressão não trabalha com o conceito de confiança e, portanto, não é possível escolher os exemplos rotulados com a maior confiança necessários no Cotraining. Nesse sentido, os autores definem o conceito de confiança na regressão como aquele regressor que tem o menor erro quadrático (MSE) no conjunto incremental dos exemplos rotulados $L$. Devido ao fato que calcular o MSE de cada regressor em todo o conjunto em cada iteração seria muito custoso, os autores propõem uma aproximação dada pela Equação 3.16, onde $h$ denota o regressor original e $h^{\prime}$ o regressor refinado pelo exemplo não rotulado novo. Para cada exemplo $x_{u}$, o algoritmo identifica os $k$ vizinhos rotulados mais próximos e os utiliza para calcular o MSE, sendo que o regressor com a maior confiança é aquele que maximiza o valor de $\delta\left(x_{u}\right)$. Em um trabalho posterior Zhou e Li (2007) apresentam um maior fundamento teórico e estendem os experimentos, mostrando que o Aprendizado Semi-supervisionado baseado em Desacordo pode ser aplicado tanto na tarefa de classificação quanto na tarefa de regressão. A proposta desta dissertação é focada na tarefa de classificação, utilizando as duas visões teste-rede e rede-teste, as quais são independentes da medida de distância utilizada, o que é uma diferença notável com o trabalho de Zhou e Li (2007).

$$
\delta\left(x_{u}\right)=\sum_{\left.x_{i} \in k_{-} \operatorname{Vizinhos}\left(x_{u}\right)\right)}\left(\left(y_{i}-h\left(x_{i}\right)\right)^{2}-\left(y_{i}-h^{\prime}\left(x_{i}\right)\right)^{2}\right)
$$

Finalmente, Bodo e Minier (2008) fizeram um estudo de diversas abordagens do KNN e KNN semi-supervisionado para a classificação multi-rótulo, sendo que o KNN que utiliza distâncias mais sofisticadas consegue melhorar o desempenho do classificador KNN. 


\section{Classificação Semi-Supervisionada Baseada em Desacordo Usando KNN Global e Local}

\subsection{Considerações Iniciais}

Dentro dos esforços devotados para suprir a falta de informação inerente ao processo de classificação semi-supervisionada, surgiu a classificação semi-supervisionada multi-visão, detalhada no Capítulo 2. Um dos principais problemas existentes em tal abordagem de classificação é a necessidade de se ter disponíveis diferentes conjuntos de atributos provenientes de recursos distintos, o qual reduz muito a sua aplicabilidade em problemas reais. Nesse contexto, tornam-se importantes as propostas que utilizam outros recursos para a geração das visões a partir de um conjunto de atributos e que ao mesmo tempo cumpram os pressupostos necessários para um bom comportamento dos algoritmos multivisão.

Entretanto, como já comentado no Capítulo 3, é possível obter diferentes definições de vizinhos mais similares de um exemplo. Aproveitando a base teórica dada pelo Aprendizado Semi-supervisionado baseado em Desacordo, é possível combinar diversas estratégias objetivando melhorar o desempenho da classificação.

Neste Capítulo, é apresentada a proposta deste trabalho que visa auxiliar o processo de classificação semi-supervisionada utilizando duas estratégias diferentes para obter os conjuntos de vizinhos mais similares. Essas estratégias são combinadas dentro de um cenário no qual existe somente uma visão ou um conjunto de atributos disponível. A idéia é aproveitar os algoritmos multi-visão, especificamente o Cotraining, com o objetivo de combinar diversas estratégias para recuperar os vizinhos mais próximos de um exemplo. Dessa forma, o algoritmo consegue trabalhar em domínios não multi-visão, sendo a sua aplicabilidade mais abrangente. 
A seguir, essas duas estratégias são explicadas em detalhes, bem como são analisadas, sob uma perspectiva de redes KNN, que provê o desacordo entre as duas estratégias de determinação do conjunto de vizinhos mais próximos para um processo satisfatório de ajuda na classificação.

\subsection{Estratégias de Recuperação de Vizinhos}

Muitos trabalhos tentaram modificar o conceito de distância envolvido dentro do cálculo de similaridade, como já mencionado no Capítulo 3. Entretanto, a idéia principal desses trabalhos é modificar a proposta tradicional do KNN. Nesta seção, um análise da estratégia tradicional é apresentada e, posteriormente, será descrita uma estratégia alternativa que considera a distribuição global dos dados, segundo a proposta feita por Motta e Lopes (2009). Além disso, é apresentada evidência teórica do desacordo entre as duas estratégias para um bom desempenho no cenário do Aprendizado Baseado em Desacordo.

\subsubsection{KNN Local}

O KNN Local é a estratégia tradicional para classificar um novo exemplo com base em $k$ vizinhos mais próximos do mesmo. Dentro do processo de obtenção dos $k$ vizinhos mais próximos, a estratégia tradicional efetua apenas uma consideração local ao exemplo a ser rotulado. Em outras palavras, recuperam-se aqueles exemplos que, na perspectiva do novo exemplo, encontram-se mais próximos dele. O KNN Local utiliza esta vizinhança obtida para a predição do rótulo do novos exemplos. Por exemplo, tendo a distribuição ilustrada na Figura 4.1, diferentes novos exemplos a serem rotulados consideram vizinhanças distintas. Considerando a distância euclidiana como base, pode-se definir a vizinhança de um exemplo como todos os exemplos que se encontram dentro da hiperesfera no espaço $n$-dimensional com raio igual à distância do elemento mais afastado dentro dos $k$ vizinhos mais próximos.

Uma busca linear pode ser utilizada para encontrar os $k$ vizinhos mais próximos de um novo exemplo com complexidade $O(l)$, sendo $l=\left|X_{L}\right|$. No Algoritmo 4 é mostrada uma possível implementação do KNN Local. Nesta implementação, é utilizada uma heap para guardar os $k$ vizinhos que contenham as menores distâncias de cada exemplo, obtendo a complexidade linear se $k<l$. A complexidade do algoritmo pode ser diminuída utilizando-se tipos de dados especializados para particionamento do espaço que sejam capazes de atingir uma complexidade logarítmica, como as $k d$-trees, citadas no Capítulo 3. Embora a complexidade em tempo seja diminuída, a complexidade em memória aumenta proporcionalmente com a quantidade de atributos, sendo que o desempenho para dimensões muito grandes é pior do que a busca sequencial em cenários complexos (Weber et al., 1998). Por esse motivo, é importante fazer uma análise criteriosa para a escolha da implementação do KNN Local.

Considerando um cenário com poucos dados rotulados, é provável o aumento do erro 


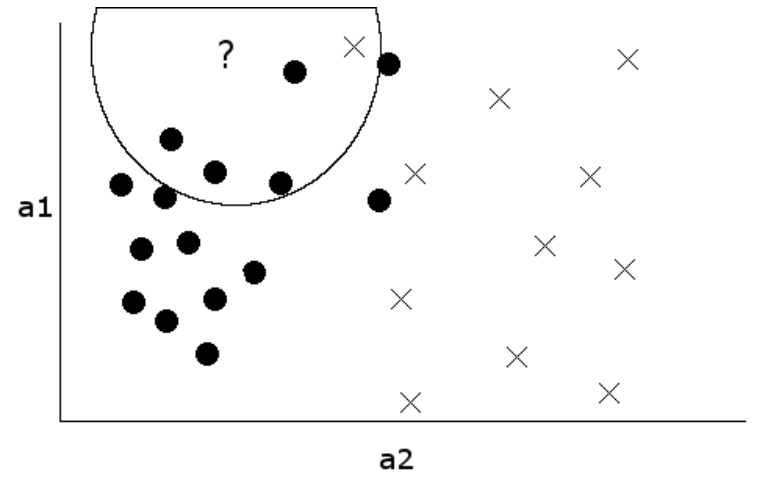

(a)

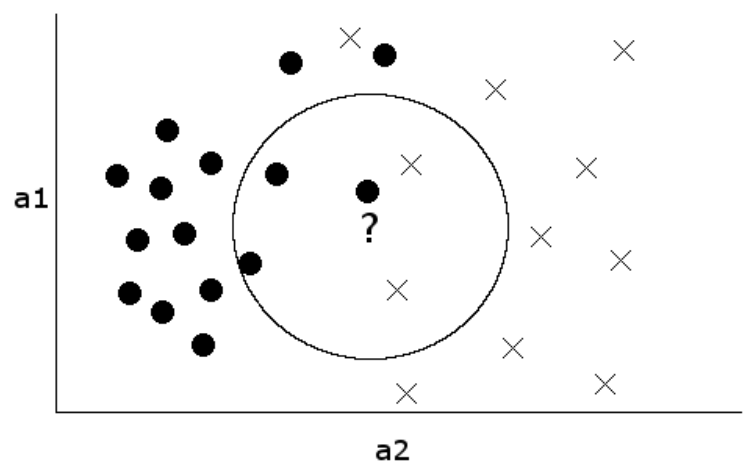

(c)

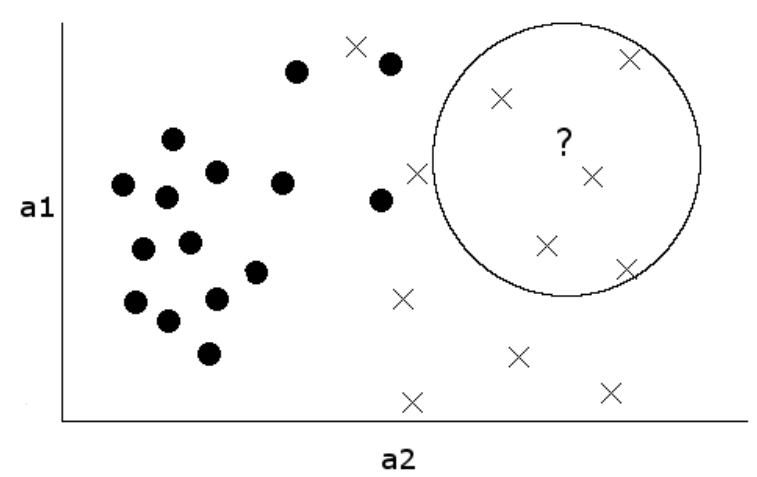

(b)

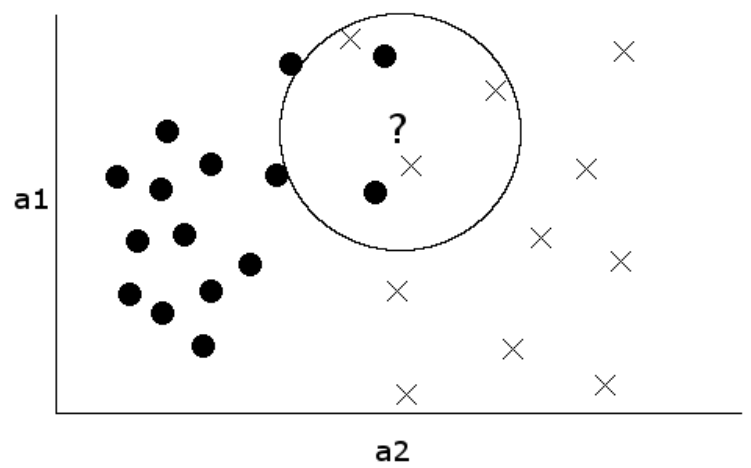

(d)

Figura 4.1: Diferentes exemplos novos a serem rotulados o KNN Local. Os exemplos em 4.1a e 4.1b são rotulados com confiança alta, pois a grande maioria de vizinhos pertencem a mesma classe. Entretanto, os exemplos mostrados em 4.1c e 4.1d são rotulados com uma confiança menor.

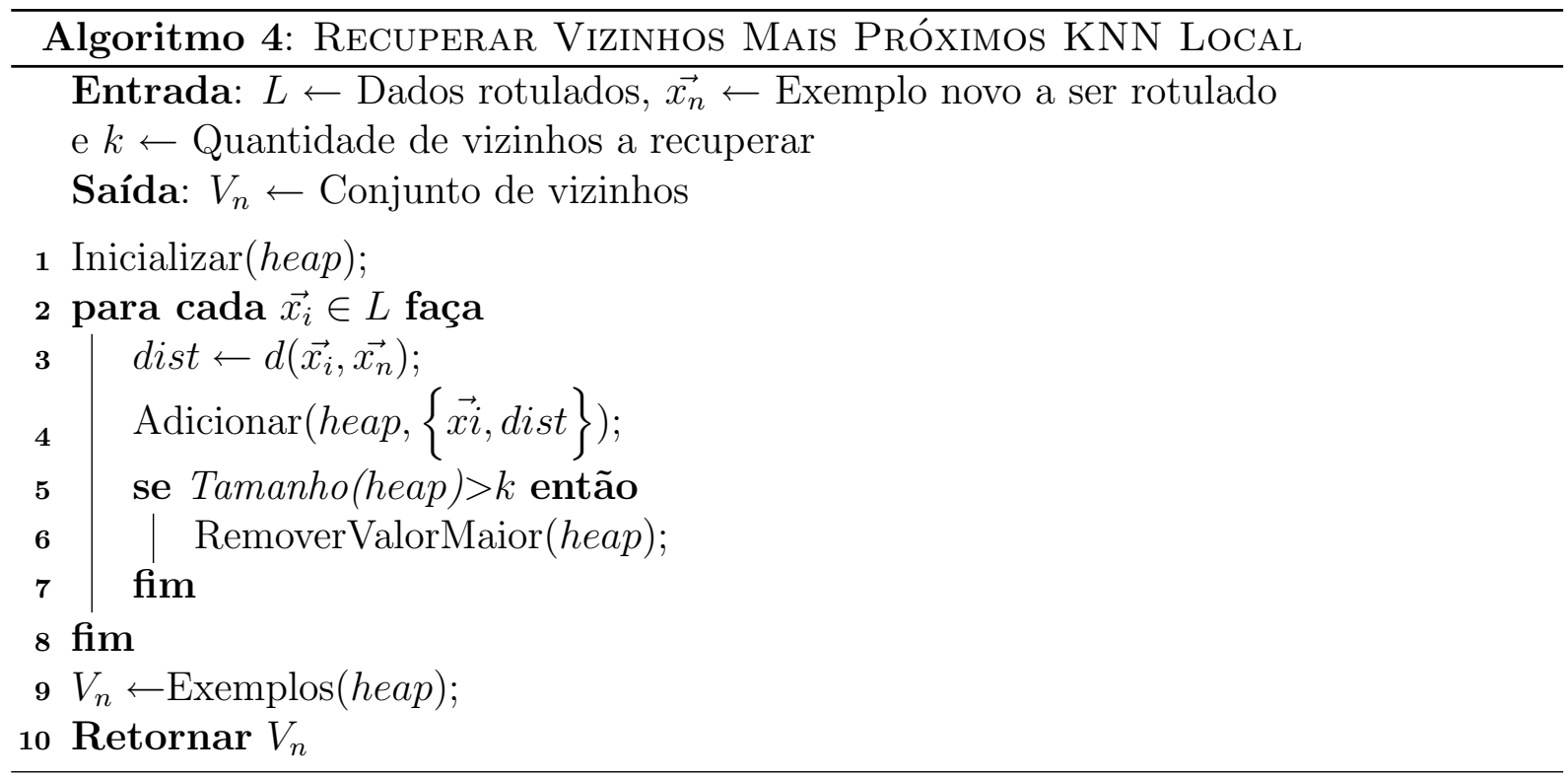

cometido pelo KNN Local devido ao fato que, inicialmente, a pouca quantidade de exemplos não representa a distribuição real das classes, ocasionando erros de classificação, especificamente, na etapa de treinamento de algoritmos semi-supervisionados. Por exemplo, no caso da utilização do algoritmo Selftraining para treinar semi-supervisionadamente 
um classificador KNN, existe a possibilidade de degradar o aprendizado, adicionando ao conjunto de treinamento exemplos rotulados de forma errada. Visando atacar esse problema, uma outra estratégia para a recuperação dos vizinhos mais próximos é proposta a seguir.

\subsubsection{KNN Global}

Diferente da estratégia tradicional chamada neste trabalho de KNN Local, o KNN Global modifica a perspectiva de similaridade para recuperar os vizinhos mais próximos de um exemplo. Assim, a estratégia utilizada no KNN Global indica que os vizinhos mais próximos de um exemplo $\overrightarrow{x_{n}}$ são aqueles exemplos $\overrightarrow{x_{i}} \in X_{L}$ que contêm o novo exemplo dentro dos seus $k$ vizinhos mais próximos. Em outras palavras, os vizinhos mais próximos de $\overrightarrow{x_{n}}$ são aqueles que são influenciados pela aparição do novo exemplo na distribuição dos dados. No capítulo anterior foi introduzido tal conceito, especificamente na construção de uma rede especial conhecida como redes KNN. Neste trabalho, pretende-se aproveitar esta estratégia para obter um conjunto de vizinhos mais próximos que contenha informações diferentes da abordagem tradicional. Devido ao fato de que para obter esta nova vizinhança é necessário fazer uma análise global dos exemplos, neste trabalho, essa estratégia foi chamada de KNN Global. Na Figura 4.2 é mostrado o conjunto de vizinhos obtidos utilizando as duas estratégias para visualizar melhor as possíveis diferenças entre as estratégias para o valor de $k=5$. Pode-se notar que os conjuntos de vizinhos podem ser parecidos, como mostrado na Figura 4.2a; iguais, como mostrado na Figura 4.2b; ou totalmente diferentes como mostrado na Figura 4.2c. Ressalta-se que com esta estratégia, a quantidade de vizinhos mais próximos de um exemplo não é necessariamente $k$, podendo tomar valores entre $k \in\{0 . . N\}$, sendo $N$ o número total de exemplos rotulados.

Uma implementação inicial desta estratégia faz uma busca exaustiva sobre o conjunto de dados para encontrar os vizinhos mais próximos. No Algoritmo 5 é mostrada a implementação desta estratégia com a busca linear. Nesse algoritmo, são recuperados os $k$ vizinhos de cada exemplo do conjunto $L$, e são armazenados aqueles que contêm o novo exemplo dentro dos seus $k$ vizinhos mais próximos. A complexidade total do algoritmo é $O(l \times(l+k))=O\left(l^{2}\right)$, assumindo que $l \gg k$. Era esperado que o custo computacional deste algoritmo fosse maior do que a complexidade do KNN Local uma vez que para calcular os vizinhos mais próximos com o KNN Global é necessário invocar várias vezes o KNN Local para recuperar os seus $k$ vizinhos mais próximos.

Embora o KNN Global apresente uma alta complexidade para recuperar vizinhos, esta estratégia consegue, em alguns casos, recuperar conjuntos de vizinhos diferentes do que o KNN Local. Esta característica sugere que pode existir uma sinergia que favoreça o desempenho final dos classificadores. Porém, é necessário verificar se essa diferença entre os classificadores é relevante para a sua combinação com o algoritmo Cotraining, especificamente, dentro do contexto do Aprendizado Baseado em Desacordo. Por esse motivo, o desacordo entre as duas estratégias de recuperação de vizinhos é analisado com 

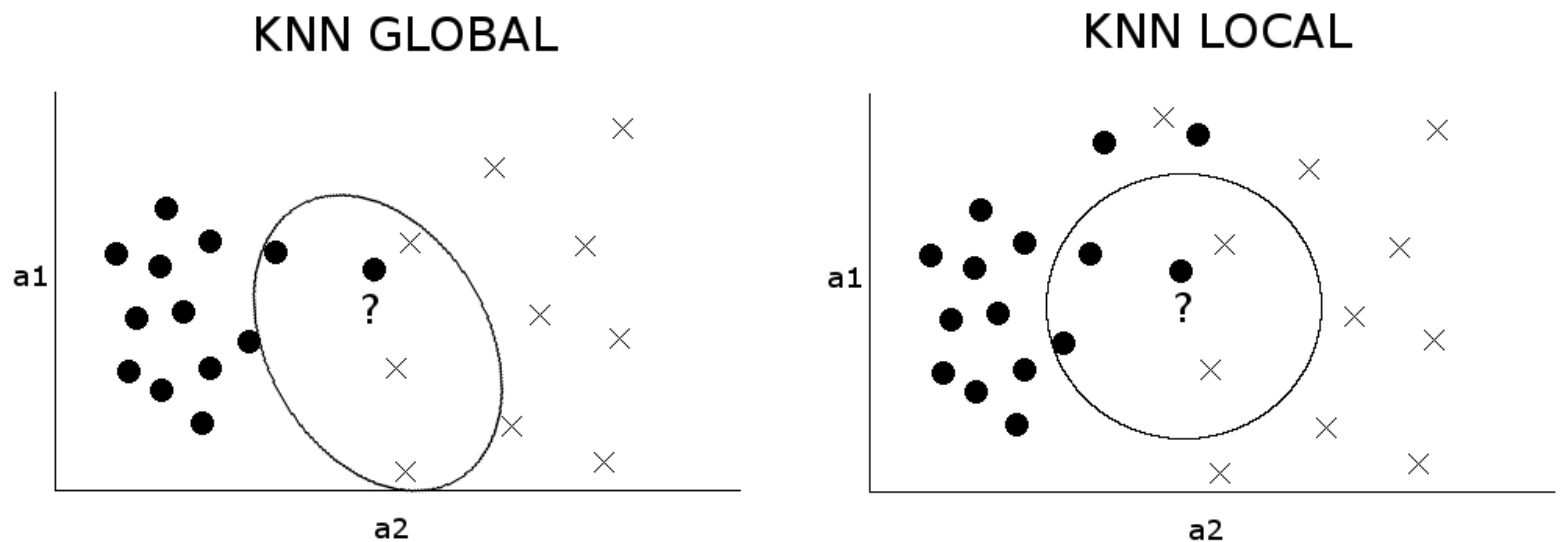

(a)
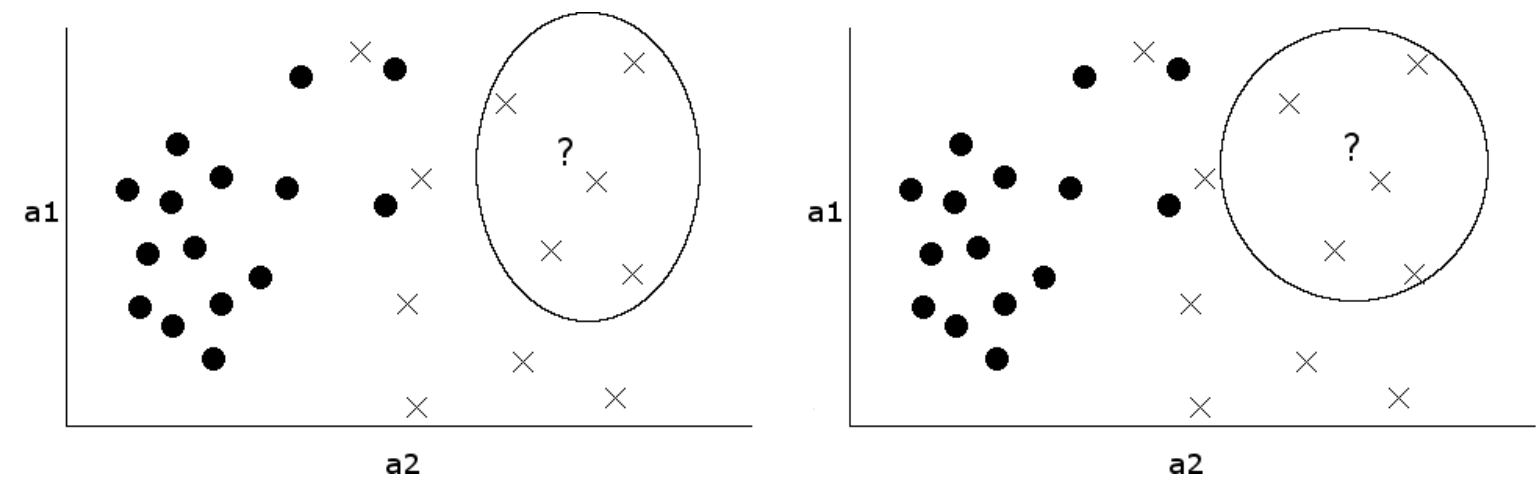

(b)
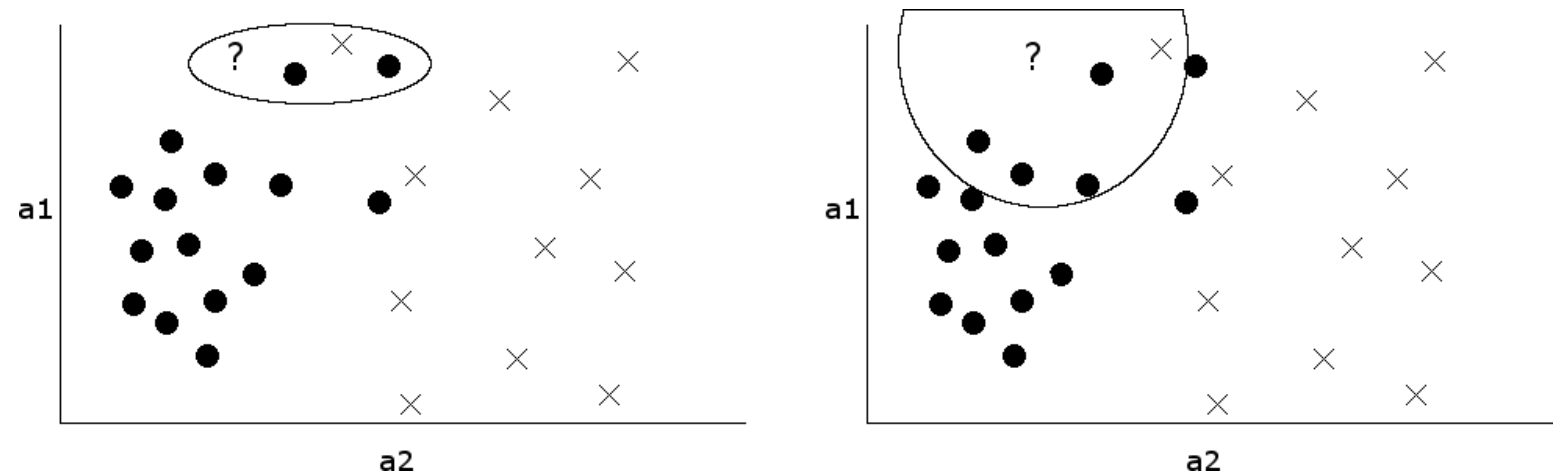

(c)

Figura 4.2: Conjunto de vizinhos para diferentes exemplos segundo a estratégia KNN Global, com $k=5$.

mais detalhe a seguir.

\subsubsection{Análise de Desacordo entre as Estratégias}

A diferença entre o KNN Global e Local pode ser analisada sob a perspectiva das redes KNN, já introduzidas no Capítulo 3, da seguinte maneira. Define-se $G_{k}$ como a rede KNN gerada utilizando o conjunto de treino $X_{L}$. Considera-se $G_{k}$ como um grafo direcionado, ou seja, as arestas de saída do vértice $v_{i}$ representam a conexão de tal vértice com os seus $k$ vizinhos mais próximos. Já as arestas de entrada ou incidentes, conectam o vértice $v_{i}$ com aqueles vértices que contêm a $v_{i}$ dentro dos seus $k$ vizinhos mais próximos. Dessa maneira, 


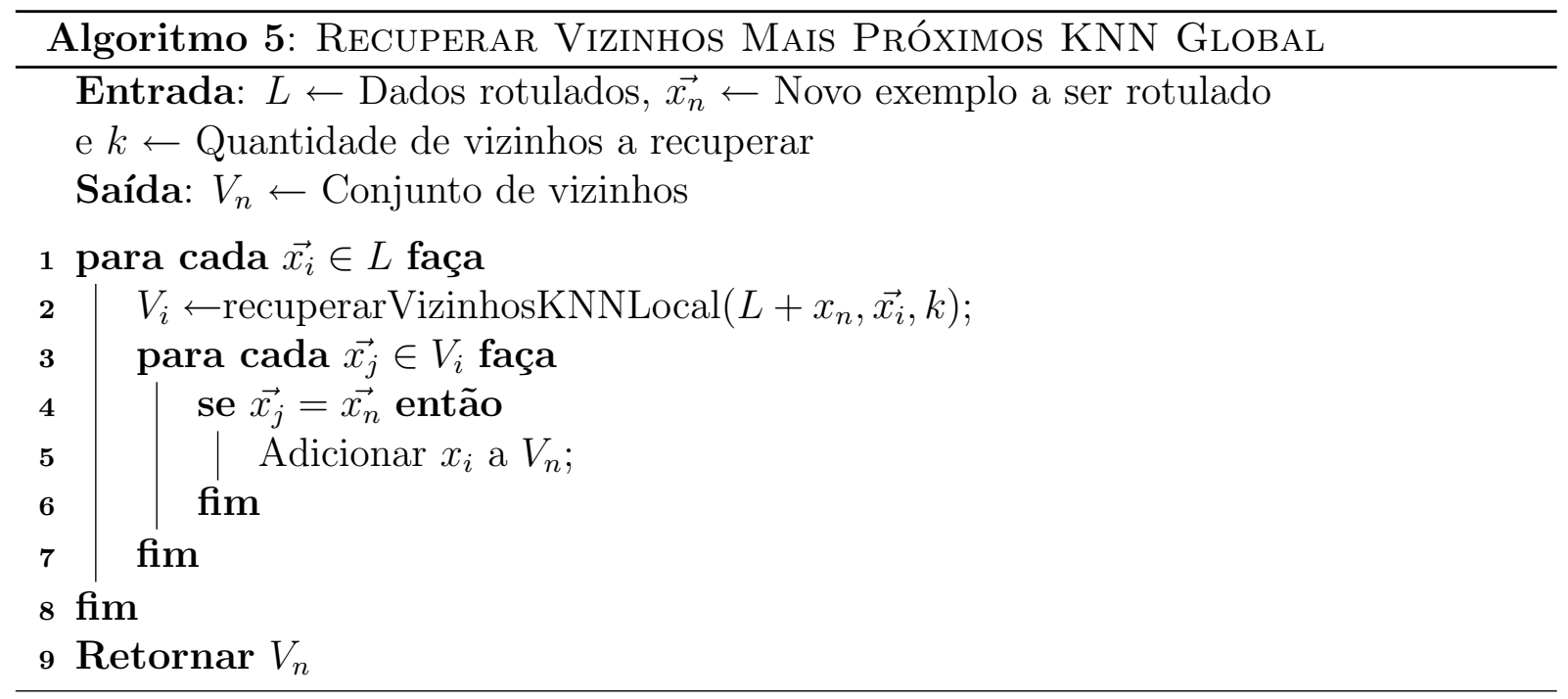

quando um novo vértice $v_{\text {new }}$ necessita fazer parte do grafo, a distribuição global de arestas de $G_{k}$ mudará. Com isso, após o processo de re-distribuição de arestas, encontrar os $k$ vizinhos mais próximos de $v_{\text {new }}$ torna-se um processo trivial. Como mostrado na Figura 4.3, com o KNN Local os vizinhos mais próximos de " $\square$ " com $k=3$ são $\{1,2,3\}$ e com o KNN Global são $\{1,2,3,5\}$. Note-se que as arestas bidirecionais representam as conexões com os vértices compartilhados pelas duas estratégias.

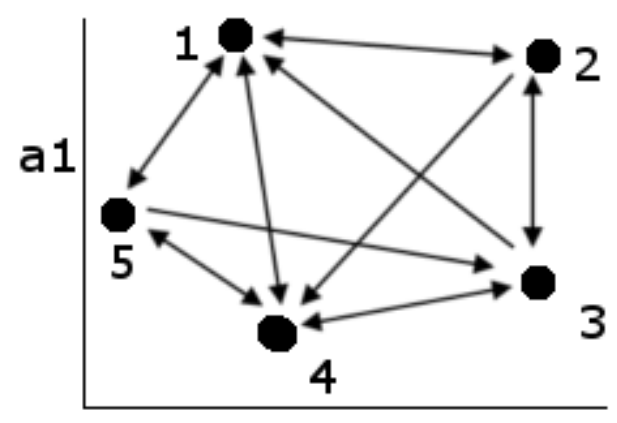

a2

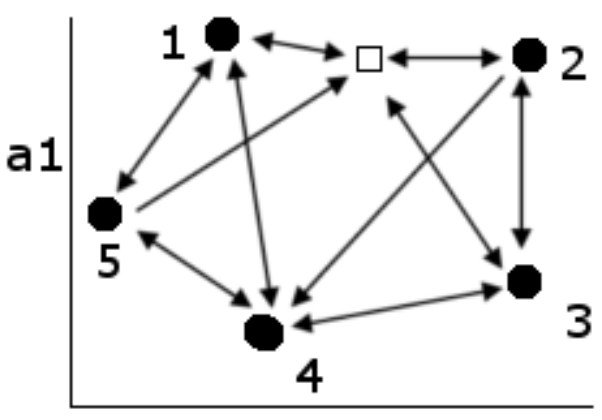

a2

Figura 4.3: Re-distribuição de arestas em uma rede KNN com $k=3$. O quadrado representa o novo vértice $\vec{x}_{n e w}$.

Considerando as estratégias tradicionais para predizer os rótulos, da mesma forma como é feita na votação majoritária ou votação ponderada, podem-se definir dois cenários diferentes. O primeiro cenário ocorre quando a distribuição de dados em torno de $v_{\text {new }}$ tem uma única classe ou uma grande classe majoritária $C$. Neste cenário é muito provável que as duas estratégias concordem. Para provar esta afirmação, definamos a distribuição de dados em torno de $v_{\text {new }}$ como $S D$, sendo $|S D| \gg k$. Com isso, a vizinhança Vlocal $_{k}$ encontrada pelo KNN Local é um subconjunto de $S D$ e, portanto, a classe predita será a classe $C$ com uma alta confiança. Por outro lado, com a estratégia KNN Global, a vizinhança encontrada $\mathrm{Vglobal}_{k}$ pode conter elementos mais distantes do que o KNN Local, mas sempre mostrando uma proximidade relativa ao novo exemplo $v_{\text {new }}$. Assim, 
assume-se que Vlocal $_{k} \wedge V$ global $k \subseteq S D$ e, portanto, que a classe predita por classificadores baseados nas duas estratégias concordaram com alta probabilidade.

O segundo cenário é dado quando a distribuição de dados $S D$ tem classes diferentes. Tal cenário é frequente nas fronteiras de decisão. Nesse sentido, espera-se que a classe predita ou a confiança de classificação seja distinta nestes casos, pois é bem provável que os conjuntos de vizinhos não compartilhem vértices. Na perspectiva de redes KNN, espera-se que as arestas não bidirecionais permitam gerar o desacordo necessário para um bom processo de ajuda conjunta dos classificadores. Tal desacordo é mais notório quando a dispersão dos dados varia entre classes próximas. Como mostrado na Figura 4.4, o aparecimento de um novo vértice terá maior impacto na distribuição das arestas de saída de um componente quando sua conexão aos vértices do componente altera pouco a dispersão atual. Consequentemente, se uma classe é suficientemente compacta, o aparecimento de um novo vértice não influenciará na distribuição das arestas de saída de tal classe.
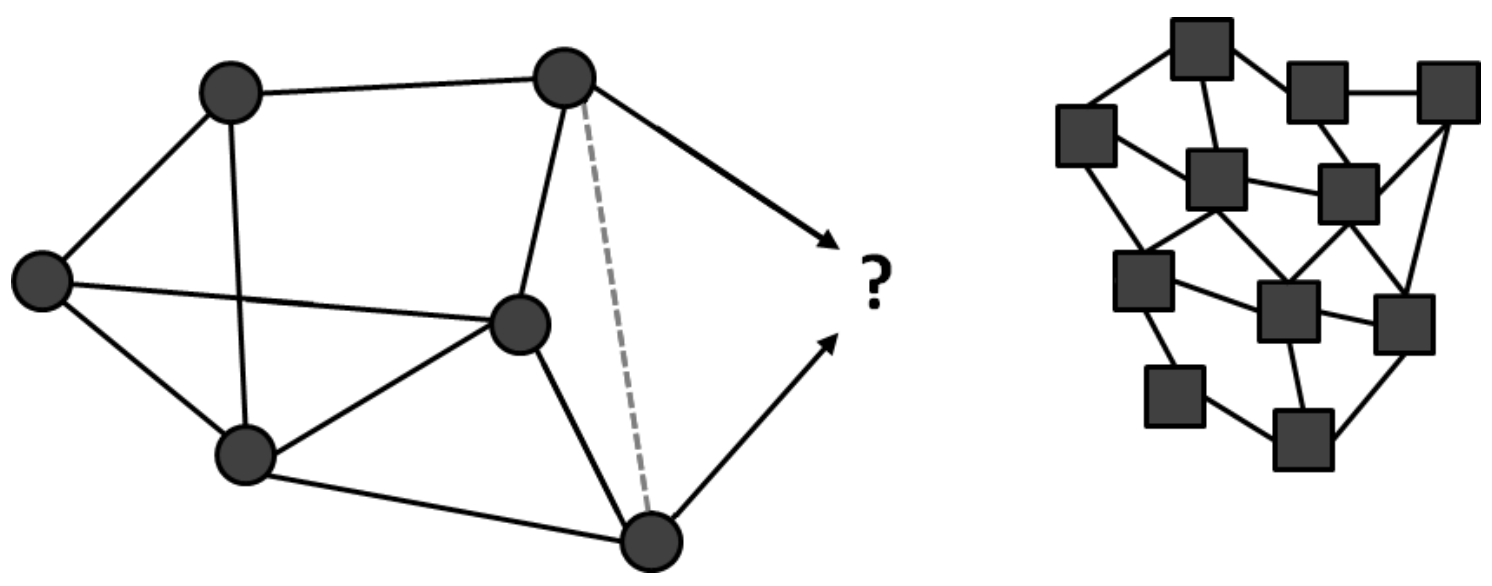

Figura 4.4: Impacto do novo exemplo na distribuição de arestas de classes esparsas e compactas.

A condição necessária para que a aparição de um novo vértice influencie um vértice $v_{i}$ pode ser definida formalmente como: dado $N k\left(v_{i}\right)$ como os $k$ vizinhos mais próximos de $v_{i}$, existirá uma aresta de $v_{i}$ para $v_{\text {new }}$ se

$$
d\left(v_{i}, v_{\text {new }}\right)<\operatorname{argmax}\left(d\left(v_{i}, v_{j}\right)\right)_{v_{j} \in N k\left(v_{i}\right)}
$$

Portanto, enquanto o KNN Local define uma maior importância à perspectiva do novo exemplo para decidir a qual classe ele pertence, o KNN Global dá maior importância a topologia da rede, o que pode ser entendido como a perspectiva global para definir uma confiança de classificação do novo exemplo. Este fato sugere que a combinação destas estratégias significará uma melhora no desempenho da classificação, especialmente, nas fronteiras de decisão. Nesse sentido, a combinação dessas duas estratégias se apresenta como uma idéia interessante, pois espera-se um processo de ajuda nos casos em que uma estratégia tenha problemas durante a rotulação de um novo exemplo. A seguir, é apresentada a proposta para a combinação dessas estratégias, dentro de um cenário no qual existem poucos dados rotulados disponíveis para o treinamento dos classificadores. 


\subsection{Combinando o KNN Local e Global com Cotraining}

O algoritmo Cotraining mostra-se como uma alternativa interessante para a combinação das duas estratégias apresentadas anteriormente. Com isso, embora os pressupostos tradicionais do Cotraining indique que é necessário duas visões explícitas nos atributos, como mostrado no Capítulo 2, estudos recentes mostram que é possível combinar classificadores com diversidade suficiente aproveitando o fundamento teórico fornecido pelo Aprendizado Baseado em Desacordo. Nesse contexto, neste trabalho foi proposta uma alternativa para o problema da falta de informações disponíveis dentro do aprendizado semi-supervisionado. Para tal objetivo, utilizou-se unicamente uma visão explícita nos atributos e, em seguida, foram gerados dois classificadores, sendo o primeiro baseado no KNN Local e o segundo baseado no KNN Global. Tais classificadores foram combinados com o algoritmo Cotraining.

\subsubsection{Metodologia}

A metodologia proposta neste trabalho, como mostrado na Figura 4.5, assume que existem inicialmente pequena quantidade de dados rotulados e muitos dados não rotulados. Uma vez disponível, dois classificadores baseados nas estratégias Global e Local são gerados. Então, o algoritmo Cotraining é utilizado para o "treinamento" dos classificadores. Nesse treinamento semi-supervisionado, o conjunto de dados rotulados é incrementado iterativamente com os exemplos rotulados com a maior confiança por cada classificador. Consequentemente, as superfícies de decisão de cada classificador são atualizadas a cada iteração. Após o processo de treinamento iterativo, também pode-se considerar o classificador combinado utilizando a proposta feita por Blum e Mitchell (1998), o qual é definido como o classificador final da metodologia.

Para rotular um novo exemplo, cada classificador gera a sua distribuição de probabilidades e tais probabilidades são multiplicadas para obter a distribuição final de probabilidades para o novo exemplo. É importante ressaltar que, na realidade, quando o classificador combinado for rotular um novo exemplo é necessário que os classificadores treinados com cada estratégia forneçam suas confianças de classificação.

Uma característica interessante da metodologia é que não está restrita a domínios multi-visão, e é independente da medida de distância utilizada, sendo necessário somente uma configuração prévia da medida de distância e o valor de $k$ que será utilizado para cada problema de classificação a ser resolvido. Por exemplo, se for definido um cenário de classificação de dados textuais, uma distância apropriada poderia ser a medida do Cosenno, já se for um cenário relacional, a medida de Jaccard poderia ser usada. A estratégia de predição adotada pelo classificador também é independente do algoritmo, podendo ser escolhidas diferentes estratégias, como a votação majoritária, votação ponderada, ou alguma outra estratégia de inferência final do rótulo dado o conjunto de vizinhos. 


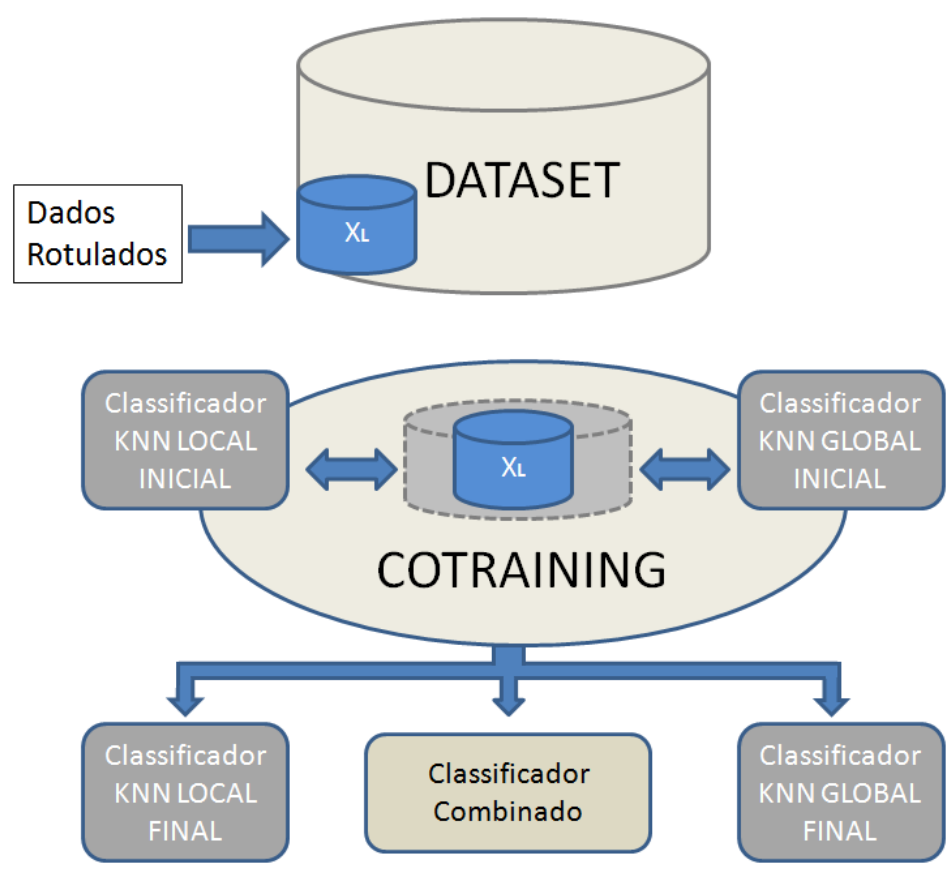

Figura 4.5: Metodologia proposta do presente trabalho.

\subsubsection{O Algoritmo Co2KNN}

O algoritmo proposto neste trabalho foi denominado Co2KNN, devido ao fato que visa integrar duas estratégias de recuperação de vizinhos do KNN com o algoritmo Cotraining. Considerando uma implementação inicial do algoritmo Co2KNN, que segue a estrutura clássica do algoritmo Cotraining, pode-se chegar em uma implementação como a mostrada no Algoritmo 6. Note-se a utilização de heaps para otimizar a seleção dos $n+p$ exemplos rotulados com a maior confiança.

Considerando tal algoritmo, a complexidade de cada iteração do Cotraining pode ser calculada como a soma de algumas etapas da seguinte forma: O(treinamento) + $O$ (classificação). As complexidades da etapa de treino são lineares, pois simplesmente geram uma lista com os dados rotulados. As complexidades da classificação estão definidas pela complexidade de recuperar os vizinhos mais próximos do exemplo novo por cada estratégia, a mesma que já foi calculada anteriormente. Considerando esses cálculos e considerando $u$ como a quantidade de exemplos não rotulados dentro do subconjunto $A$ e $l$ como a quantidade de exemplos rotulados de $L$, a complexidade da etapa do treinamento é $O(2 \times l)$ e a etapa de classificação tem como complexidade $O\left(u \times\left(l+l^{2}\right)\right)$, considerando a busca linear para o KNN Local. Ressalta-se que em cada iteração é necessário que as duas estratégias classifiquem todos os dados não rotulados e façam um ranking para escolher aqueles que foram rotulados com a maior confiança. Finalmente, a complexidade total é dada por $O\left(\right.$ loops $\left.\times\left(2 \times l+u \times\left(l+l^{2}\right)\right)\right)=O\left(\right.$ loops $\left.\times u \times l^{2}\right)$. Considerando que $l \rightarrow u$, no pior caso, a complexidade poderia ser cúbica, da forma $O\left(\right.$ loops $\left.\times u^{3}\right)$, sendo este um valor muito restritivo para a aplicação em problemas reais, sendo o KNN Global a principal causa da alta complexidade.

Com o objetivo de reduzir a alta complexidade computacional do KNN Global, uma 


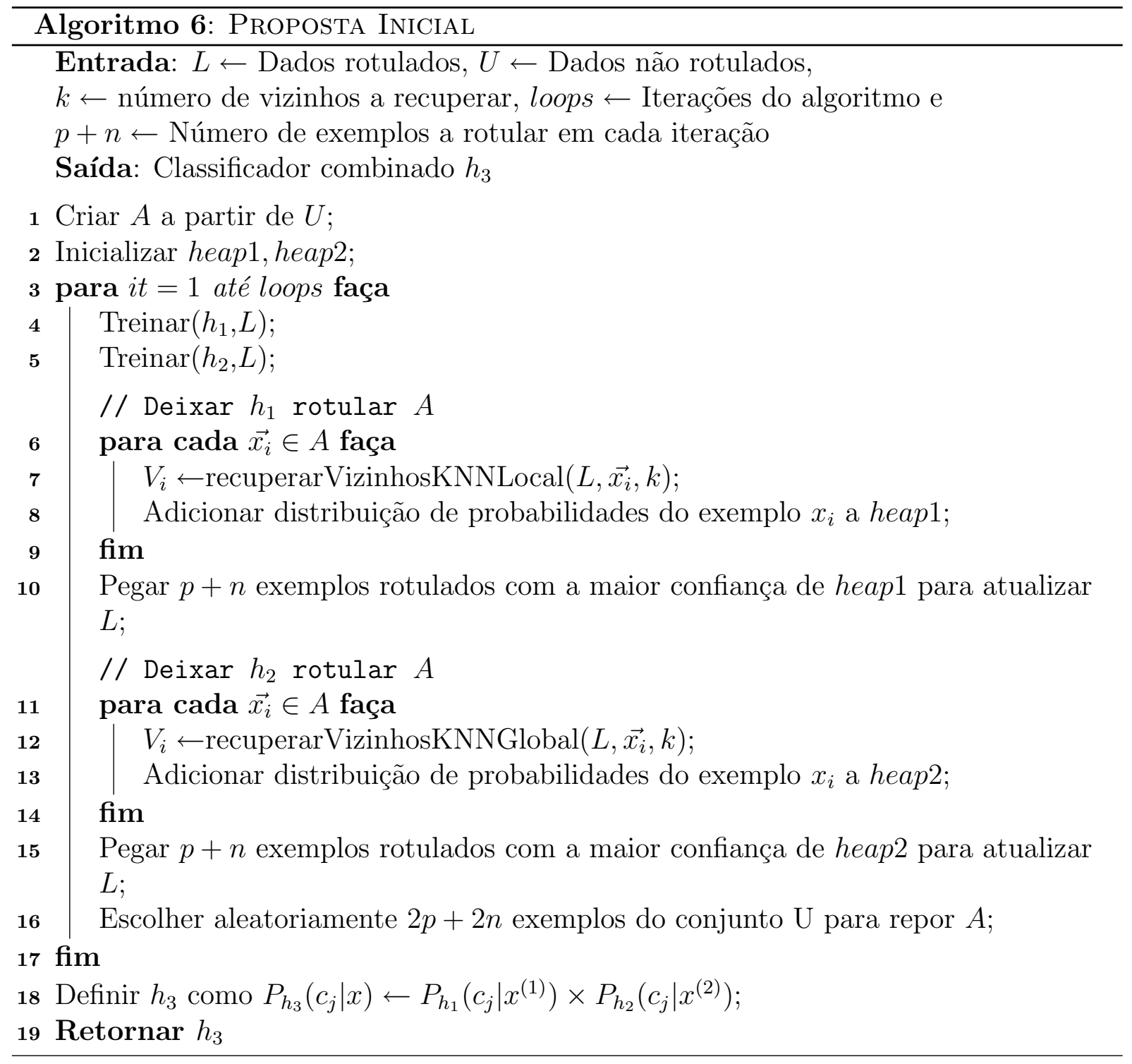

otimização da estratégia KNN Global que aproveita a característica definida pela Equação 4.1 foi proposta. Assim, uma lista de distâncias $D k_{\max }$ é gerada, a mesma que guarda a máxima distância entre cada exemplo de treinamento $\vec{x}_{i}$ e o seus $k$ vizinhos mais próximos $N k\left(\vec{x}_{i}\right)$. Com esta lista, a complexidade de recuperação da vizinhança com a estratégia KNN Global torna-se linear em relação ao número de exemplos rotulados de treinamento.

Considerando tal otimização da estratégia KNN Global, verifica-se que é possível evitar a busca exaustiva para cada exemplo do conjunto $L$. Entretanto, a complexidade de treinamento é afetada por esta otimização, pois é necessário fazer um préprocessamento na etapa de treinamento para gerar a lista com as distâncias máximas. Esse pré-processamento consiste em percorrer o conjunto de dados rotulados e calcular os valores da lista para ser utilizada na etapa de classificação. O algoritmo da etapa de treinamento para o classificador baseado na estratégia KNN Global, que envolve o préprocessamento citado, é mostrado no Algoritmo 7, sendo que para a construção da lista foi utilizada uma busca exaustiva de complexidade $O\left(l^{2}\right)$. Assume-se que é possível obter o argumento máximo da lista em $O(1)$ utilizando as variáveis necessárias. 


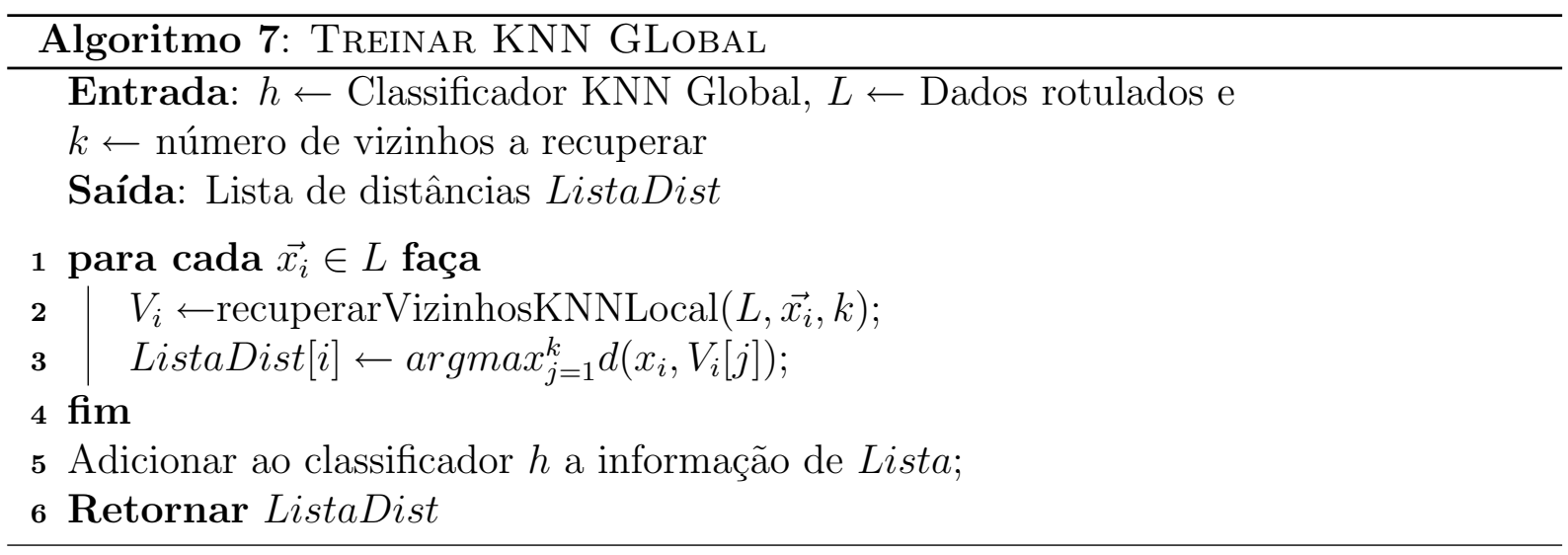

Após o treinamento, a recuperação dos vizinhos que contém o novo exemplo como um dos seus $k$ vizinhos mais próximos torna-se uma tarefa menos custosa. Como mostrado no Algoritmo 8, o único processo envolvido é percorrer a lista para verificar quais vizinhos possuem uma distância menor com o novo exemplo $x_{n}$ do que com os seus $k$ vizinhos mais próximos. Com isso, a complexidade final do algoritmo é $O(l)$, tendo um desempenho em custo computacional comparável com a complexidade da estratégia KNN Local.

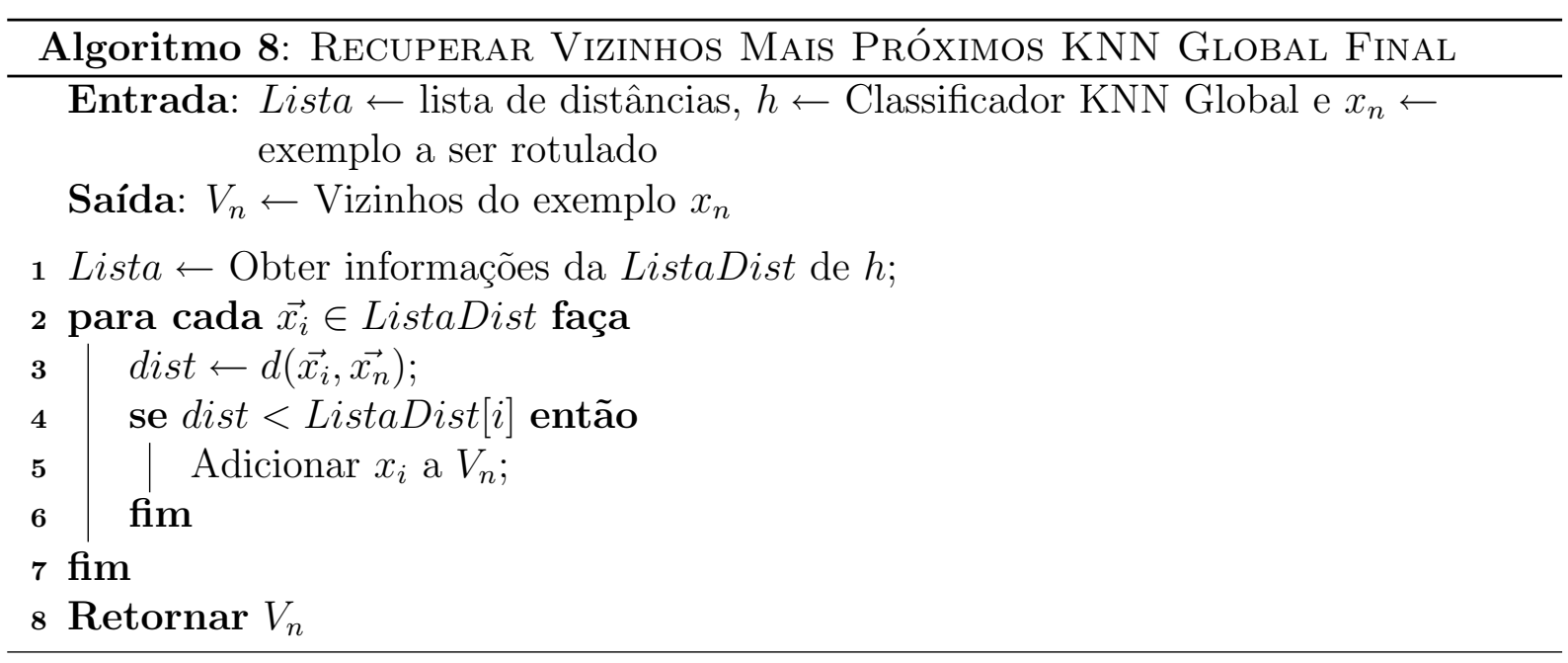

Embora a etapa de classificação torne-se menos custosa, a etapa de treinamento do classificador fica mais cara, sendo esta otimização dependente do contexto no qual a estratégia do KNN Global é aplicada. No caso do algoritmo Cotraining, tal otimização mostrou-se interessante pelo fato de que em cada iteração é necessário rotular todos os exemplos não rotulados e, com isso, pode-se obter um maior benefício global reduzindo a complexidade da classificação e aumentando a complexidade do treinamento. Finalmente, no Algoritmo 9 é mostrado a proposta final deste trabalho para a classificação de dados utilizando duas estratégias no processo de recuperação de vizinhos mais próximos. Com esta otimização, a complexidade total da proposta é $O\left(\right.$ loops $\left.\times\left(2 \times l+l^{2}+u \times l\right)\right)=$ $O\left(\operatorname{loops}\left(l^{2}+u \times l\right)\right)$, e para o caso que $l \rightarrow u$, a complexidade, no pior caso, fica quadrática, da forma $O\left(\right.$ loops $\left.\times u^{2}\right)$, sendo esta complexidade menor que a complexidade cúbica da proposta inicial. Contudo, no caso médio cumpre-se que $u \gg l$, sendo que a complexidade média do algoritmo é $O($ loops $\times u \times l)$, considerando-se tal complexidade aceitável para a 
tarefa de classificação de dados em problemas reais.

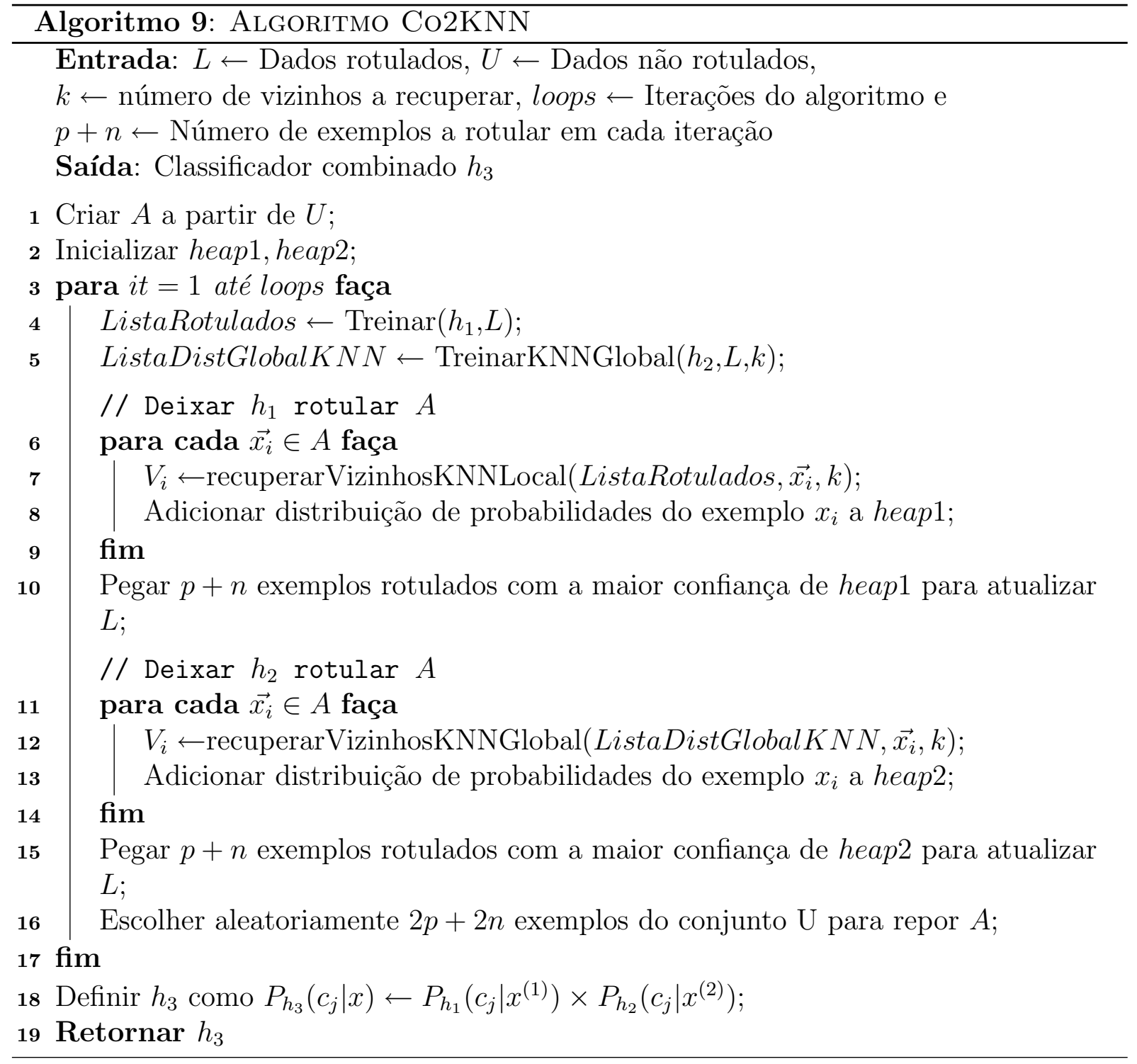

É possível otimizar mais o algoritmo aproveitando o fato de que em cada iteração o classificador tem que atualizar a lista com as maiores distâncias do conjunto L. Tal atualização poderia ser feita on-line utilizando uma estrutura adequada como uma fila de prioridade ou uma árvore $A V L$. Também é possível utilizar $k d-$ trees ou alguma estrutura de particionamento do espaço similar para reduzir ainda mais a complexidade de recuperação de vizinhos, considerando as restrições já comentadas.

\subsection{Exemplo Ilustrativo}

Com o objetivo de ilustrar mais claramente a proposta, um exemplo visual é explicado nesta seção. Considerando que a Figura 4.6 ilustra uma distribuição de dados na qual existe uma classe "vermelha" e uma "azul", sendo que inicialmente apenas uma parte dos dados está rotulada. 


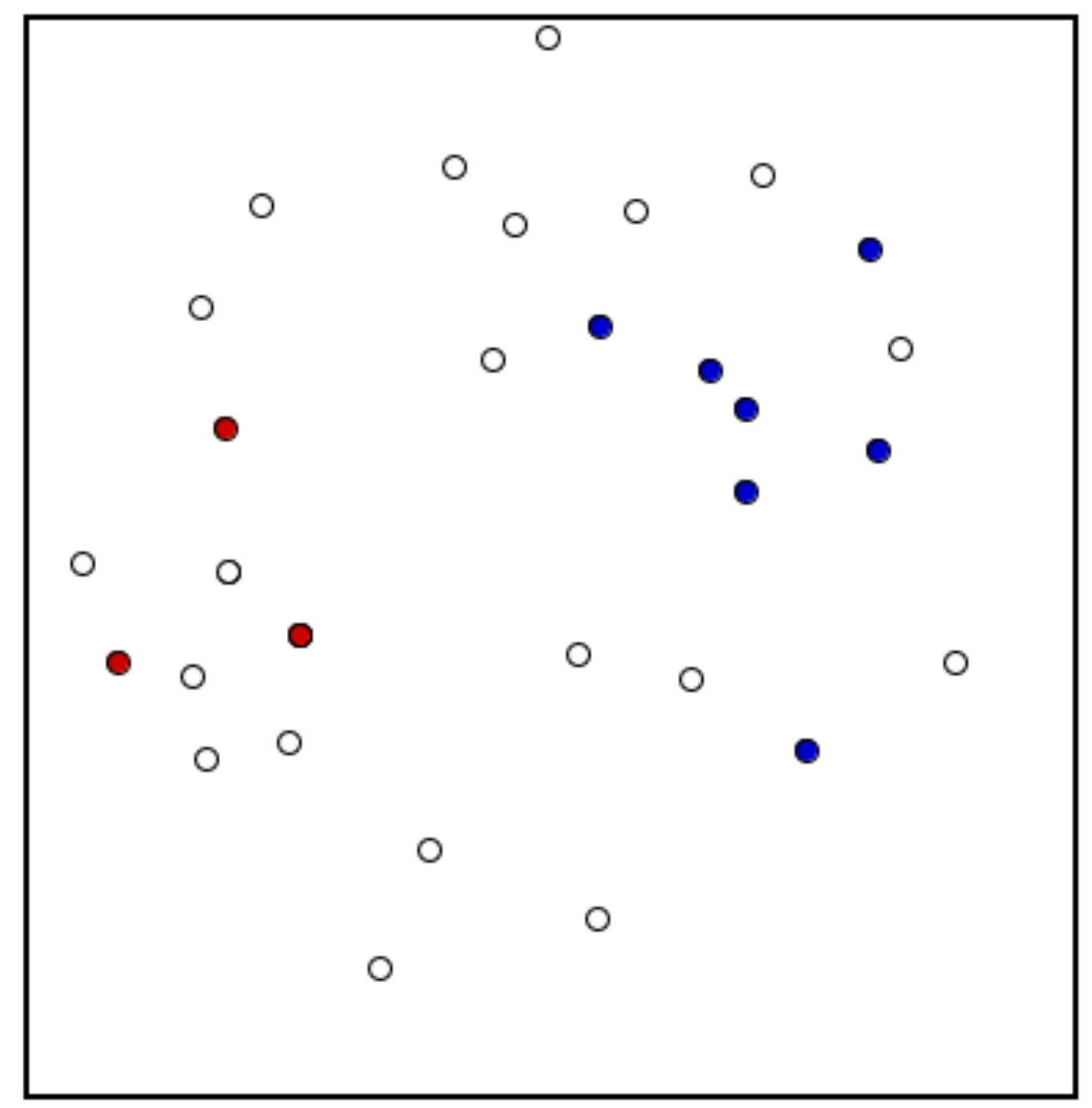

Figura 4.6: Distribuição inicial dos dados, com dados não rotulados em branco e duas classes coloridas.

O processo de treinamento com o algoritmo Co2KNN é iterativo. Em cada iteração treinam-se dois classificadores baseados nas estratégias KNN Local e KNN Global respectivamente, utilizando o conjunto atual de dados rotulados. Após a etapa de treinamento, cada classificador calcula a sua confiança de classificação para cada exemplo não rotulado, sendo os exemplos rotulados com a maior confiança selecionados para atualizar o conjunto de treino. Em cada iteração serão rotulados 2 exemplo azuis e 1 exemplo vermelho. O valor $k$ utilizado será 3 .

Após a primeira iteração, o conjunto de dados rotulados é atualizado com 3 novos exemplos, os quais são mostrados com borda preta e um número de identificação na Figura 4.7. Ressalta-se que para a recuperação de vizinhos mais próximos unicamente são utilizados os dados rotulados, adicionando a informação do novo exemplo a ser rotulado.

Para esclarecer o processo de seleção e classificação dos novos dados, cada caso será analisado separadamente. Na Figura 4.8 é ilustrado o processo de geração de arestas pelas estratégias KNN Local e Global para o exemplo 1. Como pode ser observado, as duas estratégias recuperaram o mesmo conjunto de vizinhos, concordando com alta confiança na classificação de exemplo 1 como vermelho. 


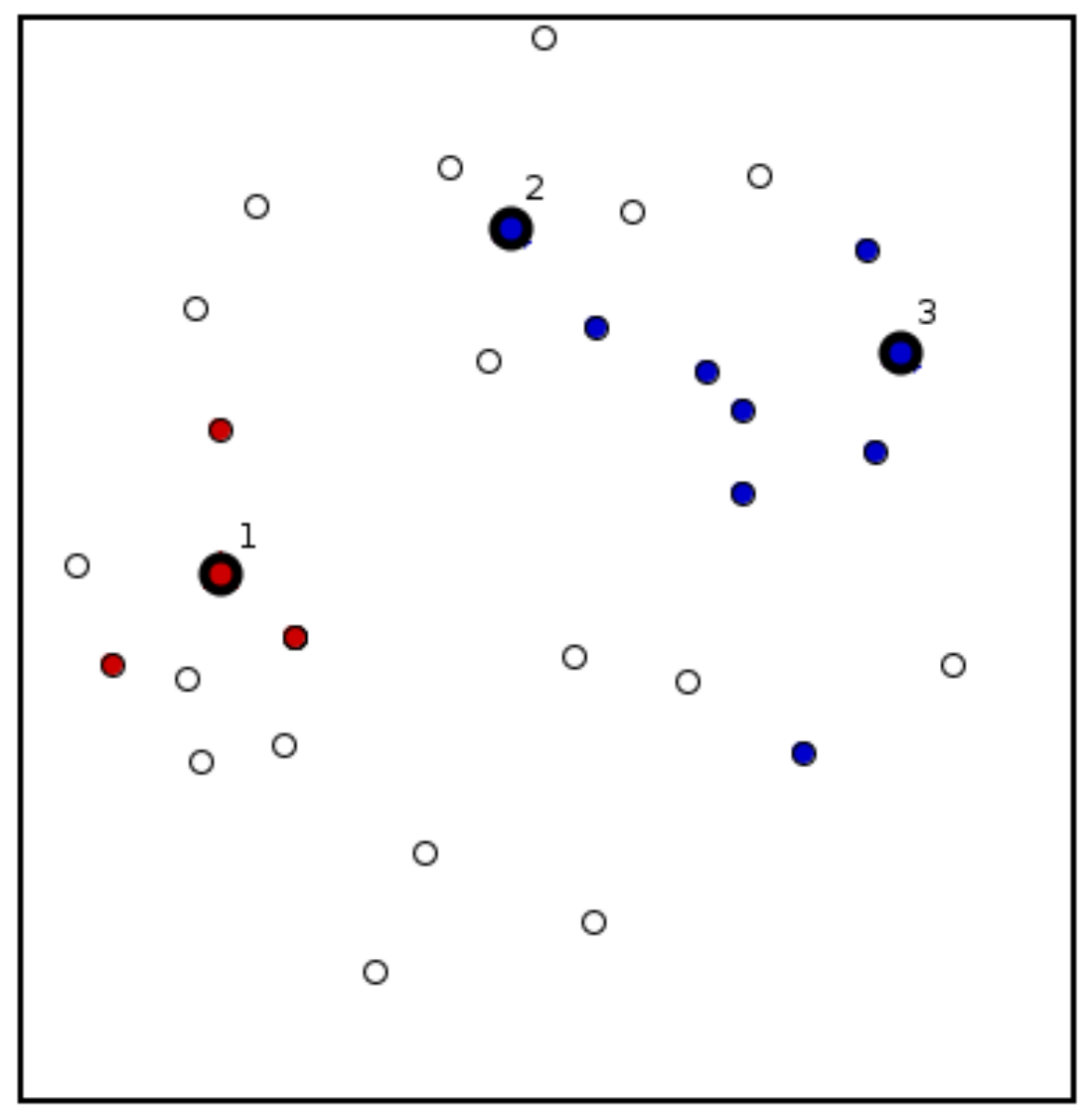

Figura 4.7: Estado do conjunto de dados após a primeira iteração do algoritmo Co2KNN.

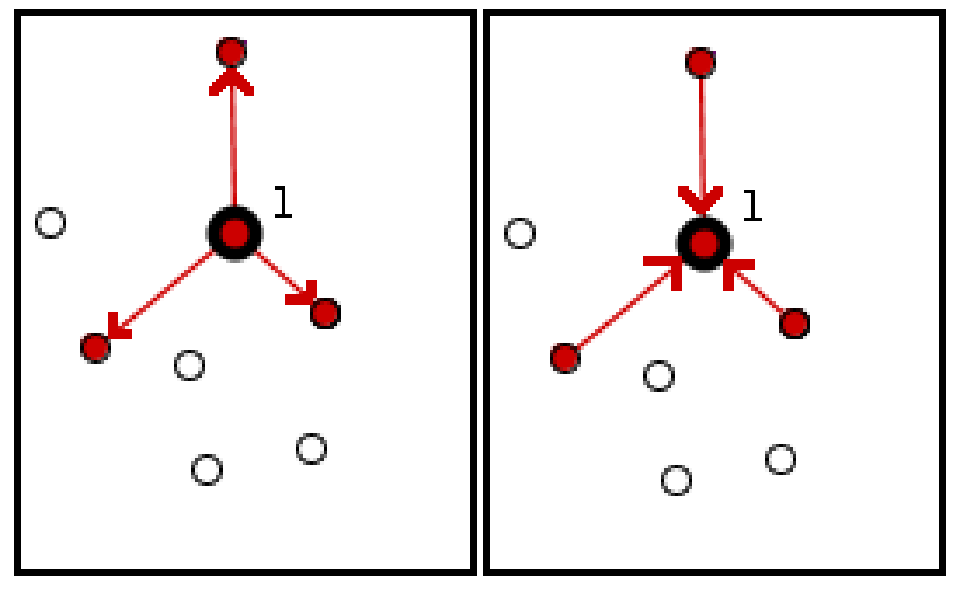

Figura 4.8: Distribuição de arestas para o exemplo 1.

Continuando o análise, na figura 4.9 é mostrada a distribuição de arestas para o exemplo 2. Note-se que enquanto a estratégia KNN Local tem uma confiança alta, a estratégia KNN Global recupera um conjunto de vizinhos com a mesma quantidade de exemplos das duas classes, considerando uma confiança igual do exemplo ser de uma classe ou da outra. Com isso, esse exemplo é considerado importante para o processo de aprendizado pois a estratégia KNN Local consegue ensinar um novo conceito à estratégia KNN Global.

Já para o exemplo 3, as duas estratégias concordam na recuperação dos vizinhos, obtendo confianças muito altas e concordando na classificação. A distribuição das arestas 


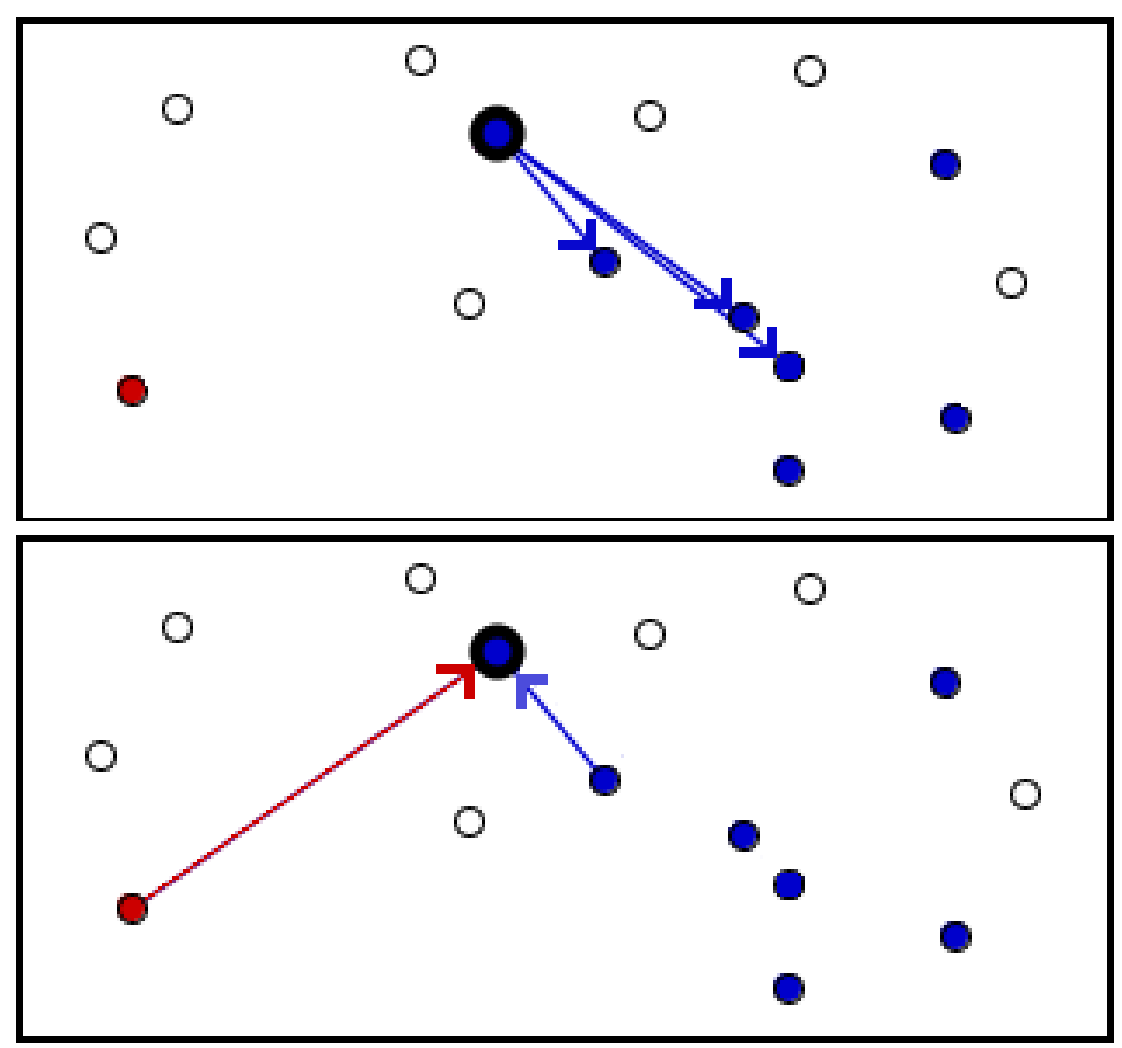

Figura 4.9: Distribuição de arestas para o exemplo 2.

para o exemplo 3 é mostrada na Figura 4.10, determinando que a probabilidade de este exemplo ser da classe "azul" é muito alta.

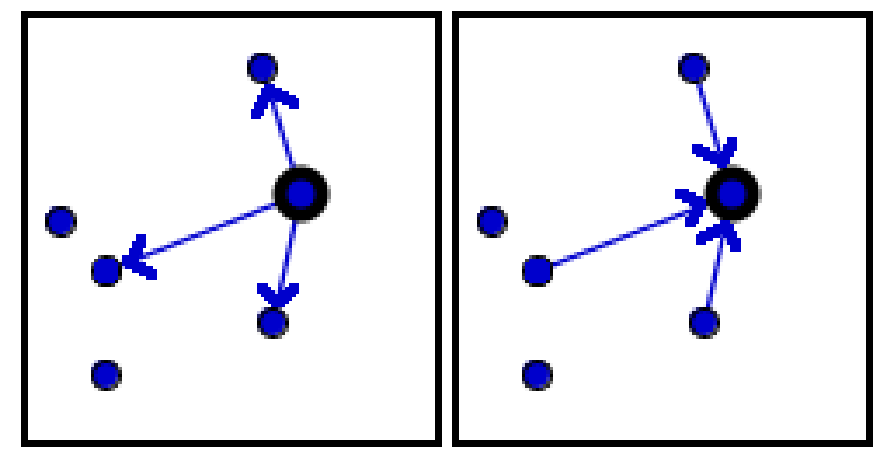

Figura 4.10: Distribuição de arestas para o exemplo 3.

Esses 3 exemplos foram selecionados porque foram os exemplos nos quais uma das duas estratégias teve a maior confiança na classificação. Consequentemente, as confianças de classificação em todos os outros exemplos não rotulados foram iguais ou menores. Nos casos de empate nas confianças, deu-se prioridade ao KNN Local para atualizar o conjunto de dados rotulados, devido a que mostrou um melhor desempenho empíricamente. Supondo que o processo continua até a iteração 4, a distribuição final dos dados seria similar à mostrada na Figura 4.11.

Finalmente, essa distribuição é utilizada pelas estratégias KNN Local e KNN Global para rotular exemplos novos fora do conjunto de treino, sendo as confianças finais calculadas multiplicando as confianças de cada estratégia, como detalhado na Seção 4.3.2. Por 


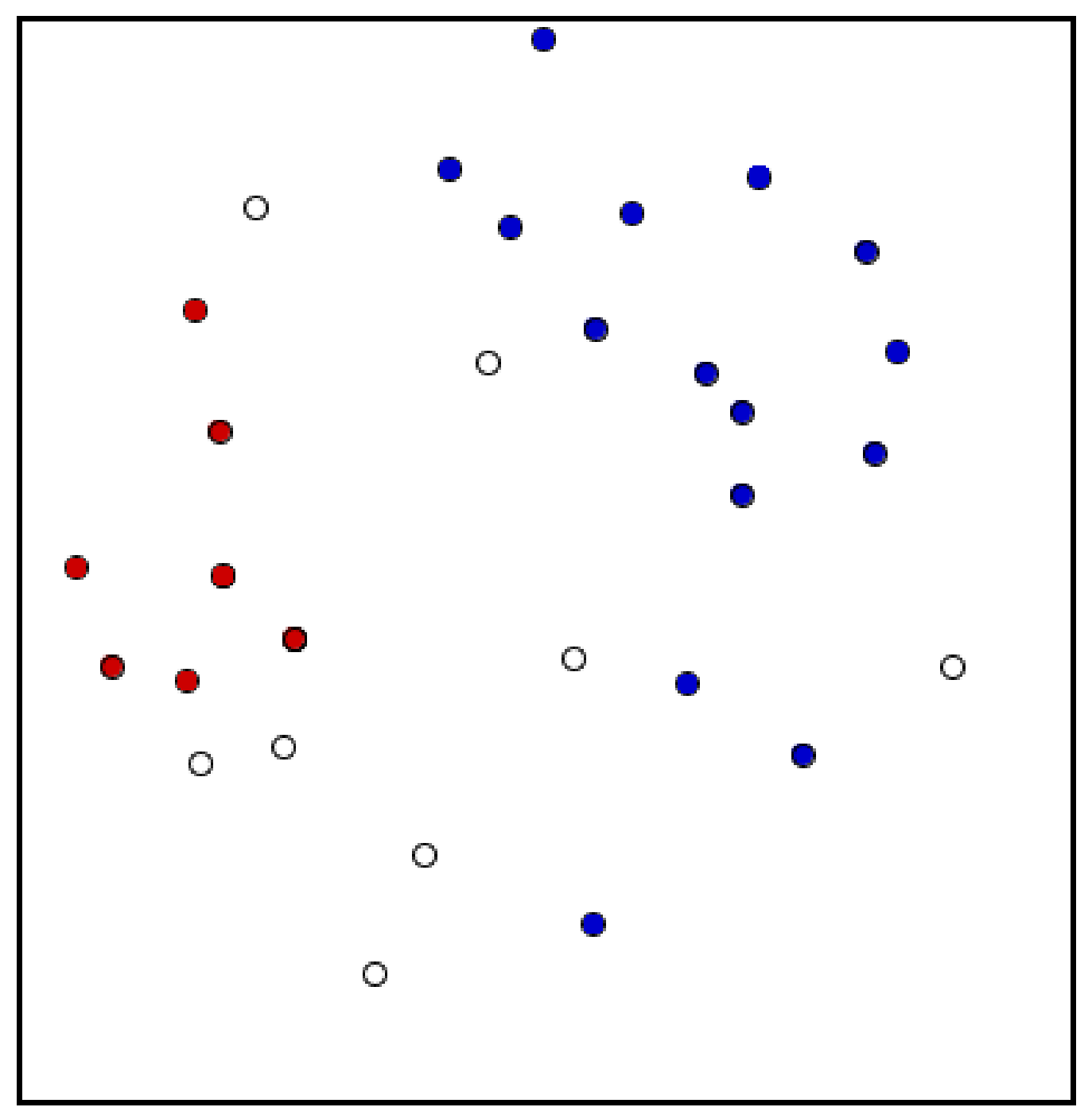

Figura 4.11: Distribuição dos dados após a iteração 4.

exemplo, se for necessário rotular o exemplo mostrado na Figura 4.12, as confianças do classificador baseado na estratégia KNN Local serão 2/3 para a classe "azul" e 1/3 para a classe "vermelha". Entretanto, para o classificador baseado na estratégia KNN Global serão 1 para a classe "azul" e 0 para a classe "vermelha". Multiplicando-se as confianças obtêm-se que a confiança do classificador Co2KNN é $2 / 3$ para a classe "azul" e 0 para a classe "vermelha", predizendo o rótulo "azul" para o novo exemplo. 


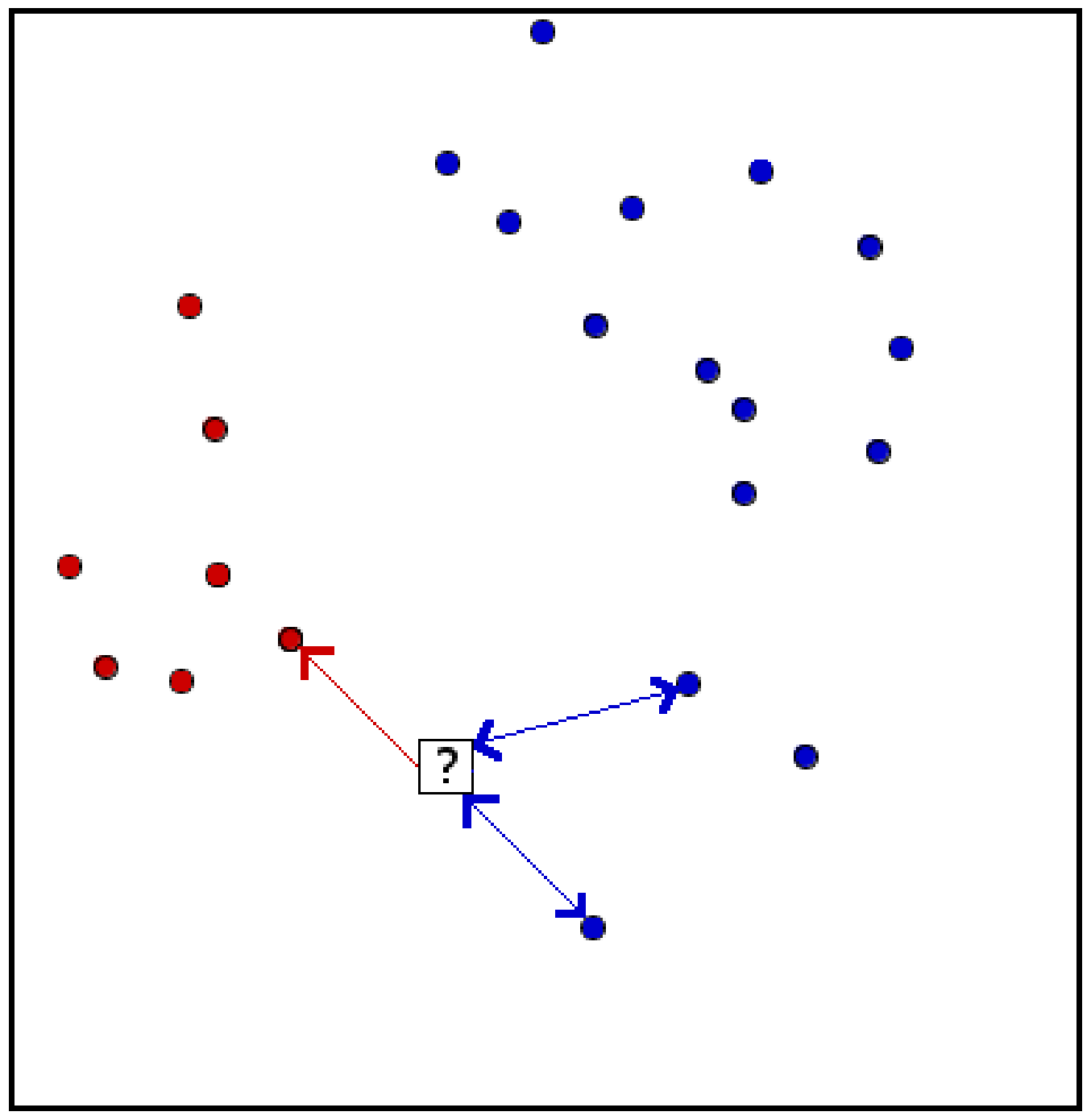

Figura 4.12: Novo exemplo a ser rotulado. 


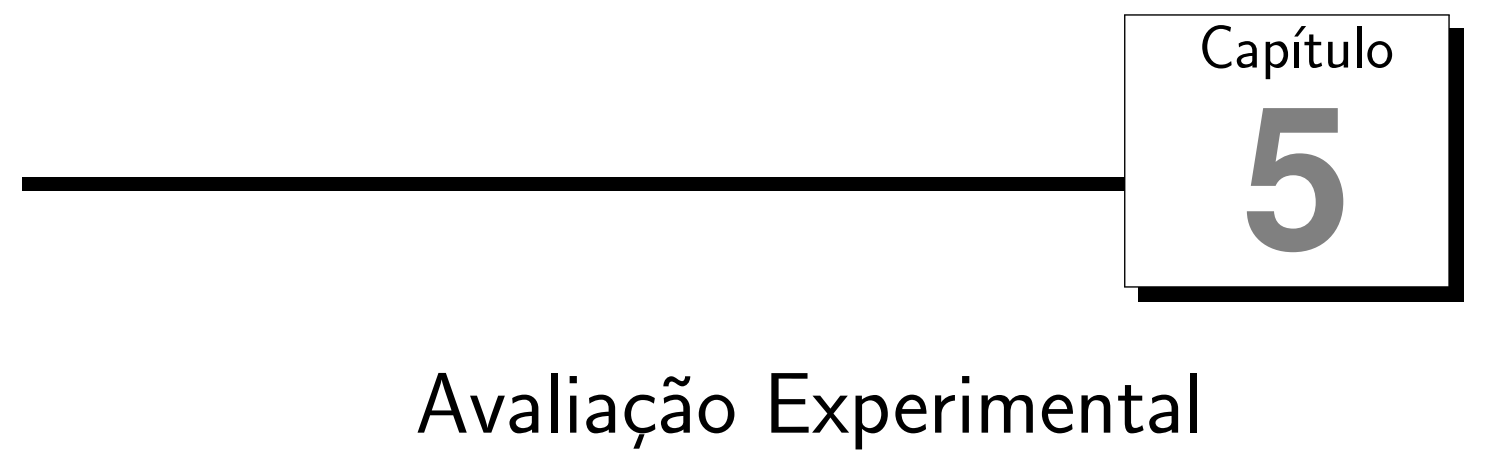

\subsection{Considerações Iniciais}

Neste capítulo são descritos diferentes experimentos que foram realizados visando avaliar o desempenho do algoritmo semi-supervisionado Co2KNN em diferentes cenários. Foram considerados cenários nos quais as bases de dados necessitam ser classificadas e poucos dados rotulados para o treinamento estão disponíveis. Adicionalmente, nesses cenários, considerou-se que unicamente uma visão dos dados nos atributos está disponível, sendo estes cenários os mais comuns em problemas reais de classificação. Em seguida, os conjuntos de dados são descritos e são detalhadas as configurações para a execução dos experimentos. Os resultados nos diferentes cenários são apresentados e analisados.

\subsection{Abordagem para Avaliação do Algoritmo Co2KNN}

O objetivo da avaliação feita foi validar que o algoritmo Co2KNN pode ser utilizado em cenários reais, já descritos previamente, e verificar se realmente as combinação das estratégias KNN Local e KNN Global, propostas no Capítulo 4, leva a uma melhora significativa na geração de classificadores. Ressalta-se que o algoritmo Co2KNN gera um classificador final combinado, o mesmo que será referido como classificador Co2KNN.

Para tais objetivos, o classificador Co2KNN foi comparado inicialmente com os principais classificadores supervisionados do estado da arte, obtendo uma base de comparação para o desempenho do Co2KNN. Posteriormente, o mesmo classificador Co2KNN foi comparado com abordagens alternativas ou variantes do KNN. Todas essas abordagens e os detalhes da comparação são explicados na Seção 5.4. Os classificadores utilizados para ser comparados com o Co2KNN foram treinados com o algoritmo semi-supervisionado Selftraining (descrito no Capítulo 2), configurado com os mesmos parâmetros do Co2KNN 
visando uma comparação mais justa.

Na Figura 5.1 é mostrada a estratégia de comparação adotada para a execução dos experimentos. Para cada base de dados, geraram-se inicialmente os 10 folds. Após a geração dos folds, o conjunto de treino foi dividido em uma pequena parte de dados rotulados e o restante foi considerado como dados não rotulados. Após o processo de geração dos conjuntos, treinou-se todos os classificadores, procurando utilizar o mesmo fold em todos os classificadores, para uma comparação mais estável e justa. Com os classificadores treinados, obteve-se o desempenho de todos os classificadores sobre o conjunto de teste, sendo este desempenho, finalmente, utilizado para a comparação entre classificadores. Como medida de comparação do desempenho foi calculado a área embaixo das curvas ROC (AUC) de cada classificador no conjunto de teste. O AUC foi utilizada como medida padrão por ser considerada uma medida mais discriminativa da qualidade da classificação do que a taxa de erro, especialmente em cenários com classes desbalanceadas (Demšar, 2006).

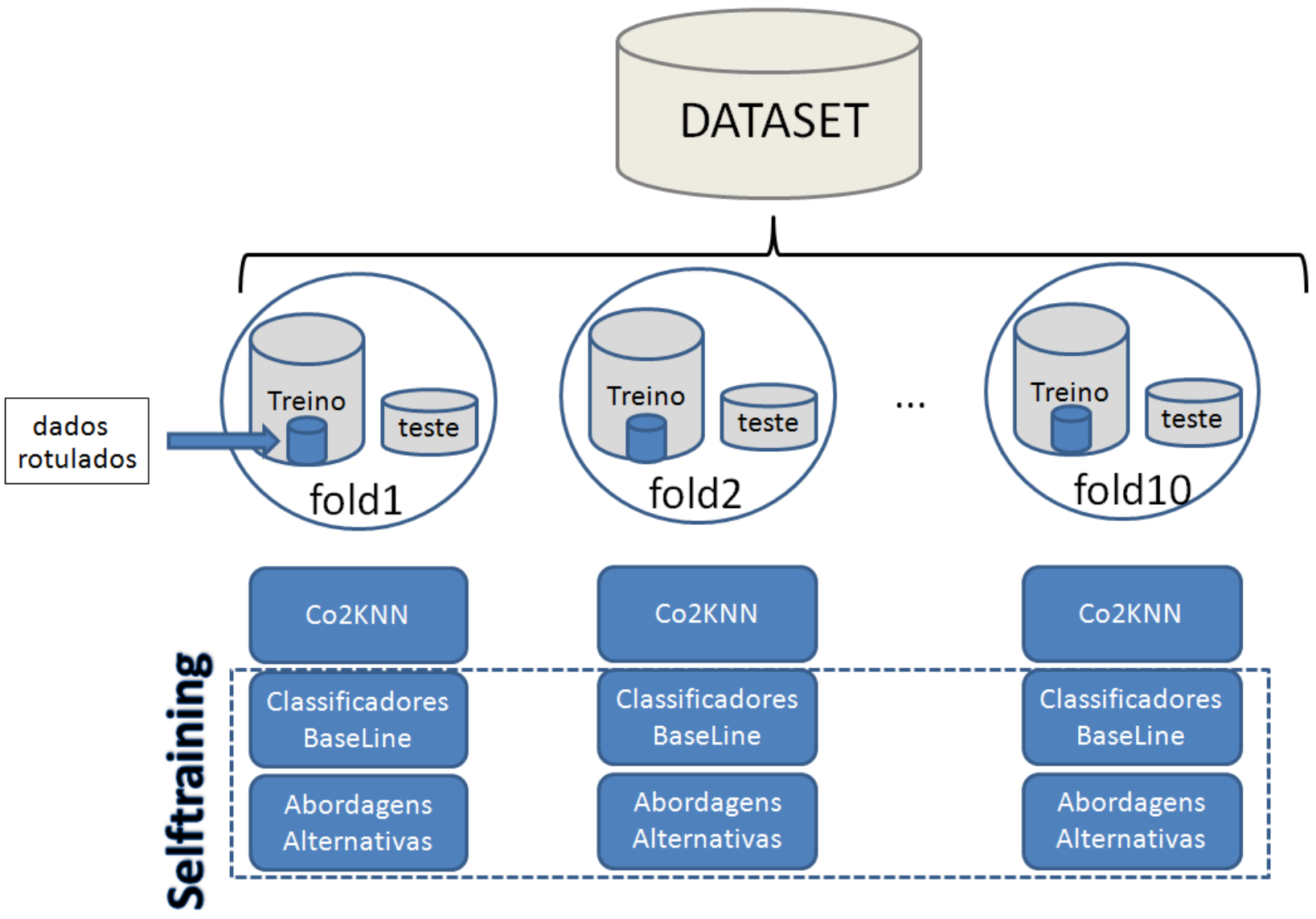

Figura 5.1: Metodologia de avaliação do presente trabalho. 


\subsection{Configurações}

\subsubsection{Conjunto de Dados}

Para a execução dos experimentos foram selecionados 17 conjuntos de dados comumente utilizados em tarefas de classificação, obtidos do repositório UCI ${ }^{1}$. Com o objetivo de simplificar a avaliação, somente conjuntos de dados com duas classes foram selecionados. Uma descrição de cada base utilizada é mostrada a seguir:

- adult: contém informações sobre salários de pessoas;

- breast-c: contém informações oncológicas para detectar pessoas com câncer;

- breast-w: contém informações oncológicas para detectar pessoas com câncer (contém atributos diferentes da base breast-c);

- colic: contém informações de cólica em cavalos;

- credit-a: contém informações financeiras de pessoas para aprovar um crédito na Austrália;

- credit-g: contém informações financeiras de pessoas para aprovar um crédito na Alemanha;

- diabetes: contém informações para detectar pessoas diabéticas;

- heart-c: contém informações médicas para detectar pessoas com doenças cardíacas;

- heart-h: contém informações médicas para detectar pessoas com doenças cardíacas (contém atributos diferentes da base heart-c);

- heart-s: contém informações médicas para detectar pessoas com doenças cardíacas (contém atributos diferentes da base heart-c e heart- $h$ );

- hepatitis: contém informações médicas para detectar pessoas com hepatite;

- ionosphere: contém informações para classificação de elétrons livres na ionosfera;

- kr-vs-kp: contém informações para predizer o ganhador de uma partida de xadrez;

- mushroom: contém informações para detectar cogumelos tóxicos;

- sick: contém informações médicas para detectar pessoas com doenças na glândula tireóide;

- sonar: contém informações de solos para detectar minas;

\footnotetext{
${ }^{1}$ http://archive.ics.uci.edu/ml/
} 
- vote: contém informações de votos de congressistas americanos para predizer se são democratas ou republicanos;

Esses conjuntos de dados foram selecionados visando manter uma variedade nos diferentes cenários de avaliação do algoritmo Co2KNN. As informações referentes à quantidade de exemplos e distribuição de classes são detalhadas na Tabela 5.1.

Tabela 5.1: Detalhe dos conjuntos de dados.

\begin{tabular}{|c|c|c|}
\hline Nome & \%Classe Majoritária & Nro. de Exemplos \\
\hline \hline adult & $76 \%$ & 48842 \\
\hline breast-c & $70 \%$ & 286 \\
\hline breast-w & $65 \%$ & 699 \\
\hline colic & $63 \%$ & 368 \\
\hline credit-a & $55 \%$ & 690 \\
\hline credit-g & $70 \%$ & 1000 \\
\hline diabetes & $65 \%$ & 768 \\
\hline heart-c & $54 \%$ & 303 \\
\hline heart-h & $64 \%$ & 294 \\
\hline heart-s & $55 \%$ & 270 \\
\hline hepatitis & $79 \%$ & 155 \\
\hline ionosphere & $64 \%$ & 351 \\
\hline kr-vs-kp & $52 \%$ & 3196 \\
\hline mushroom & $51 \%$ & 8124 \\
\hline sick & $93 \%$ & 3772 \\
\hline sonar & $53 \%$ & 208 \\
\hline vote & $61 \%$ & 435 \\
\hline
\end{tabular}

\subsubsection{Parâmetros de Entrada}

Para a execução do algoritmo Co2KNN, foram fixados os seguintes parâmetros de entrada: o número de vizinhos a serem recuperados $(k)$, o tamanho do subconjunto de dados não rotulados $(|A|)$, o número inicial de dados rotulados $\left(X_{L}\right)$ e o número de exemplos rotulados em cada iteração do Co2KNN $(n+p)$. Assim, a quantidade de iterações do algoritmo Co2KNN foi fixada em 10. O tamanho do subconjunto $A$ de dados não rotulados foi fixado a $10 \%$ do conjunto de dados não rotulados de treinamento $X_{U}$. Adicionalmente, a quantidade de dados rotulados disponíveis inicialmente foi fixada em 20, pois considerou-se que na maioria das aplicações seria viável a rotulação manual pelos especialistas de tal quantidade. Também considereou-se que o conjunto inicial de dados rotulados é estratificado. Além disso, os experimentos consideraram o número de vizinhos mais próximos $k \in\{1,3,5,15\}$. O valor máximo de $k$ foi fixado em 15 devido ao fato que nos casos em que o valor de $k$ é maior que o número de exemplos rotulados iniciais de treino, o algoritmo KNN tem uma tendência a degradar o seu desempenho. Tal degradação acontece porque em quase todos os casos a classe majoritária do conjunto rotulado será a classe predita com a maior confiança. Nesse sentido, é recomendado que 
o valor máximo do parâmetro $k$ a ser utilizado com o algoritmo Co2KNN seja menor que o número de exemplos no conjunto inicial de dados rotulados $X_{L}$.

Para a execução dos experimentos, os valores dos parâmetros $n$ e $p$ foram escolhidos dependendo da distribuição das classes, seguindo a estrutura do Cotraining tradicional. Os valores de $n$ e $p$ utilizados para todos os experimentos sobre as bases são mostrados na tabela 5.2, sendo esses valores o suficientemente pequenos para permitir uma execução eficiente do algoritmo. Embora para definir os valores de $n$ e $p$ a distribuição das classes tenha que ser conhecida previamente, é possível que esses valores sejam somente estimados por especialistas do domínio. Em outras palavras, a relação entre $n$ e $p$ não tem que ser estritamente igual à relação real entre exemplos negativos e positivos. Entretanto, quanto maior a similaridade com a relação real das classes, maior a probabilidade de conseguir um melhor desempenho com algoritmos do estilo Cotraining (Blum e Mitchell, 1998).

Tabela 5.2: Parâmetros dos conjuntos de dados no algoritmo Co2KNN.

\begin{tabular}{|c|c|c|}
\hline Nome & $\boldsymbol{n}$ & $\boldsymbol{p}$ \\
\hline \hline adult & 6 & 18 \\
\hline breast-c & 2 & 5 \\
\hline breast-w & 4 & 6 \\
\hline colic & 4 & 6 \\
\hline credit-a & 3 & 4 \\
\hline credit-g & 3 & 7 \\
\hline diabetes & 4 & 6 \\
\hline heart-c & 4 & 3 \\
\hline heart-h & 3 & 5 \\
\hline heart-s & 3 & 2 \\
\hline hepatitis & 4 & 1 \\
\hline ionosphere & 1 & 2 \\
\hline kr-vs-kp & 6 & 6 \\
\hline mushroom & 8 & 8 \\
\hline sick & 1 & 10 \\
\hline sonar & 2 & 2 \\
\hline vote & 5 & 3 \\
\hline
\end{tabular}

Com o objetivo de definir se existe diferença significativa entre o classificador gerado pelo algoritmo Co2KNN e os demais classificadores, testes estatísticos foram aplicados aos resultados. Para a comparação de "um vs muitos" foram aplicados testes não paramétricos pareados utilizando em conjunto o teste de Friedman com $p<0,05$ e o método de Dunn, como método pós-teste para possibilitar a comparação de Co2KNN com vários classificadores. Já para a comparação de "um vs um" foi utilizado o teste de Wilcoxon.

\subsubsection{Classificadores Co2KNN Utilizados}

Para a implementação do algoritmo Co2KNN, consideraram-se duas estratégias para a predição do rótulo, dado os dois conjuntos de vizinhos. A estratégia inicial, chamada de Co2KNN, define a votação majoritária para cada classificador. Por exemplo, para o 
caso mostrado na Figura 5.2, o conjunto de vizinhos recuperado com a estratégia KNN Local (arestas de saída) contém 1 vizinho da classe positiva e 2 da classe negativa. Consequentemente, a probabilidade do novo exemplo ser positivo é $1 / 3$ e de ser negativo é $2 / 3$. Entretanto, com a estratégia Global (arestas de entrada), o conjunto de vizinhos contém somente 1 vizinho da classe positiva, sendo a probabilidade, com esta perspectiva, 1 de ser positivo e 0 de ser negativo.

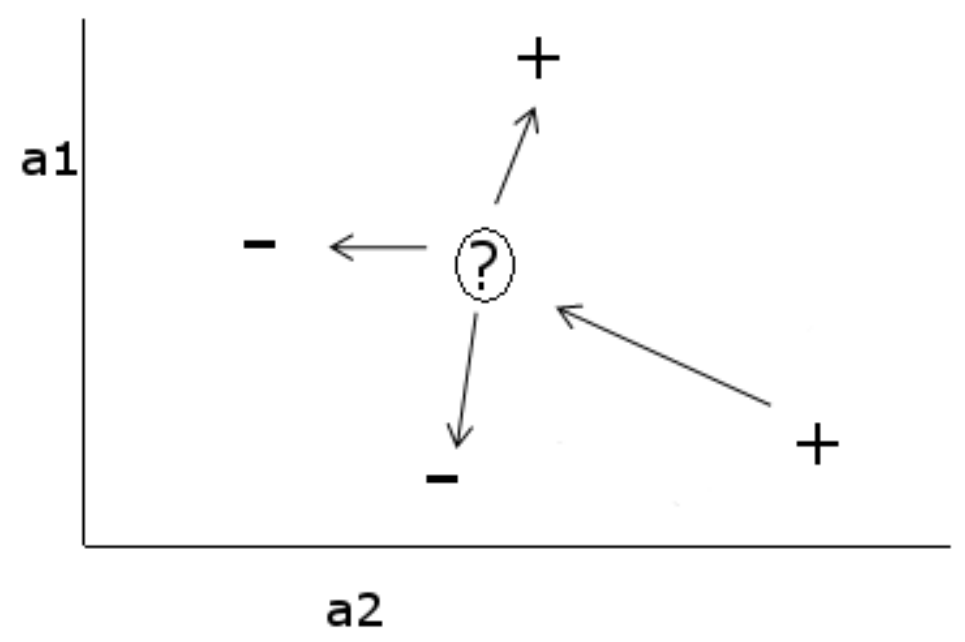

Figura 5.2: Conjunto de vizinhos recuperado com cada estratégia. O conjunto obtido pelo KNN Global está representado pelas arestas incidentes ao novo exemplo e as arestas de saída representam o conjunto obtido pelo KNN Local.

Analisando o exemplo, embora o classificador baseado no KNN Global tenha poucas arestas, ele tem uma confiança muito alta na classificação. Estes casos influenciam fortemente pelo fato que o Co2KNN atribui uma maior prioridade aos exemplos rotulados com a maior confiança. Por esse motivo, uma outra variante do algoritmo Co2KNN é proposta e utilizada. Esta variante pondera os valores das probabilidades dos classificadores multiplicando-as por um fator que representa a proporção de arestas de cada estratégia, denominado Fator de Ajuste (FA). Ou seja, tomando o exemplo anterior, existem 3 arestas da estratégia KNN Local e 1 aresta da KNN Global. O fator de ajuste para o classificador baseado no KNN Local é 3/4 e para o KNN Global é 1/4. Com isso, a probabilidade do novo exemplo ser positivo com a perspectiva do classificador baseado no KNN Local seria $(3 / 4) *(1 / 3)=(1 / 4)$ e a probabilidade de ser negativo seria $(3 / 4) *(2 / 3)=(2 / 4)$ e com o classificador baseado no KNN Global seria $(1 / 4) *(1)$ para a probabilidade de ser positivo e continua 0 a probabilidade de ser negativo. Este ajuste permite atribuir uma maior importância aos exemplos com uma maior quantidade de arestas. Ressalta-se que, com este classificador, as arestas compartilhadas ou bidirecionais são consideradas uma vez no cálculo do número total de arestas. O classificador obtido por este algoritmo será referido como Co2KNN-FA nos experimentos que serão detalhados nas seguintes seções. 


\subsection{Experimentos}

Com objetivo de gerar uma base de comparação, inicialmente foi comparado o classificador Co2KNN com classificadores tradicionais treinados utilizando algoritmo semisupervisionado Selftraining.

Após a comparação com os classificadores tradicionais, o classificador Co2KNN foi comparado com o classificador baseado no KNN Local e o baseado no KNN Global treinados separadamente com o algoritmo Seltraining. O objetivo deste experimento é mostrar empiricamente que a combinação das estratégias com o algoritmo Co2KNN é, de fato, melhor que a utilização das estratégias separadamente. Adicionalmente, foi analisada a diferença entre as estratégias KNN Local e Global, estudando em quais casos uma estratégia consegue um melhor desempenho do que a outra.

Posteriormente, o classificador Co2KNN foi comparado com a estratégia SKNN, introduzida no Capítulo 3. A SKNN pode ser considerada uma alternativa natural ao Co2KNN pelo fato que ao invés de treinar classificadores separados com os conjuntos obtidos, o SKNN propõe juntá-los para formar um novo conjunto. Também, a SKNN foi treinada com o algoritmo semi-supervisionado Selftraining. Para a implementação de todas as estratégias e algoritmos propostos, além dos algoritmos utilizados para a comparação de resultados, foi utilizado o pacote $\mathrm{WEKA}^{2}$.

Finalmente, um análise das diferenças entre os desempenhos dos classificadores Co2KNN e Co2KNN-FA é realizada.

\subsubsection{Comparação com os Algoritmos Tradicionais}

Para a comparação com os algoritmos tradicionais, o classificador Co2KNN foi configurado com diferentes valores no intervalo $k \in\{1,3,5,15\}$. Os classificadores escolhidos para a comparação foram: as Máquinas de Vetores de Suporte (com o kernel polinomial), as Árvores de Decisão (implementação do algoritmo J48) e o Naive Bayes Multinomial. Tais classificadores foram escolhidos por serem muito utilizados em diferentes tarefas de classificação e aprendizado de máquina. Os resultados para o classificador Co2KNN são apresentados na Tabela 5.3, na qual os classificadores que foram os melhores significativamente foram ressaltados. Note-se que em todos os conjuntos de dados, pelo menos alguma configuração do algoritmo Co2KNN obteve o melhor desempenho.

Para comprovar a diferença significativa entre as diferentes configurações do algoritmo Co2KNN e os algoritmos tradicionais, foi aplicado o teste de Friedman em conjunto com o método de Dunn, com $p<0,5$. Os resultados são mostrados na Tabela 5.4, sendo que as configurações do Co2KNN com $k=5$ e $k=15$ obtiveram diferença estatística com todos os classificadores tradicionais, sugerindo que o algoritmo Co2KNN melhora o seu desempenho com o valor de $k$ mais alto adotado.

\footnotetext{
${ }^{2}$ http://www.cs.waikato.ac.nz/ml/weka/
} 


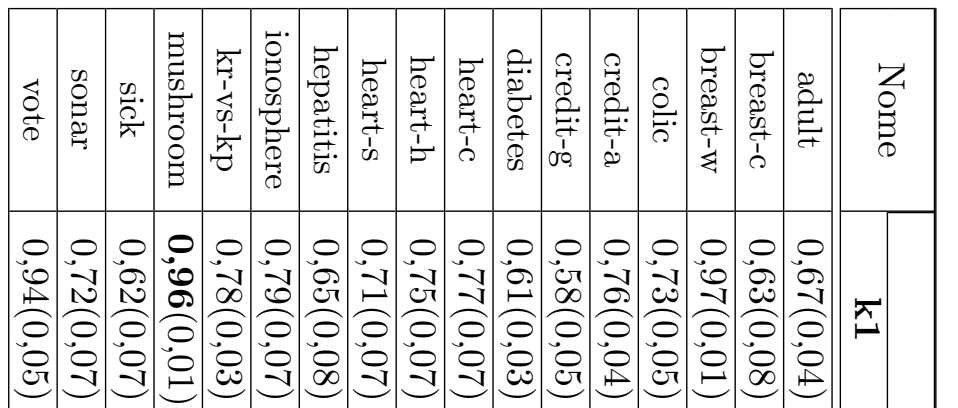

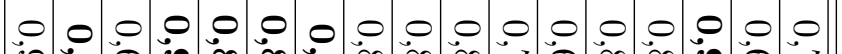

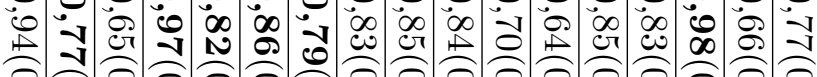

共

它谓

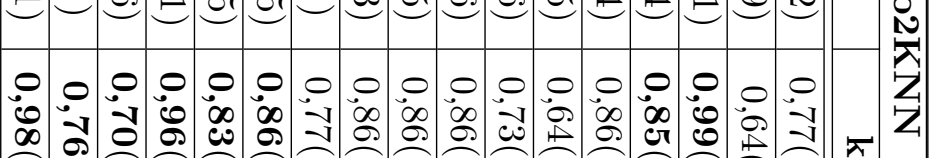

○े

思

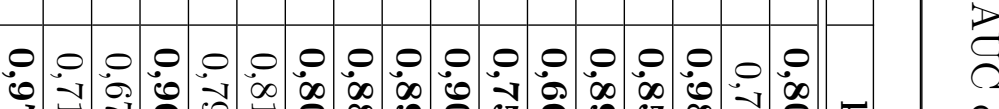

光

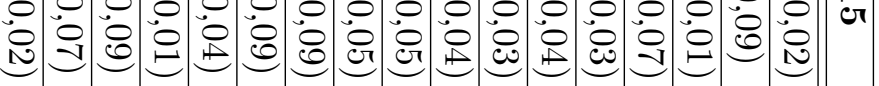

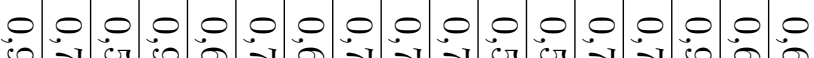

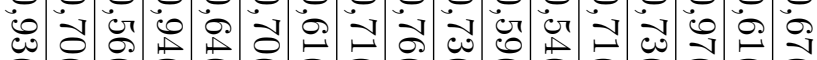

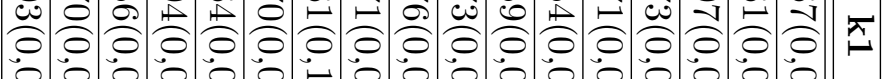

:

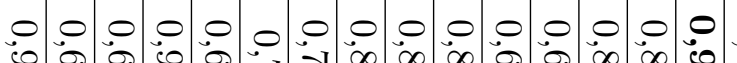

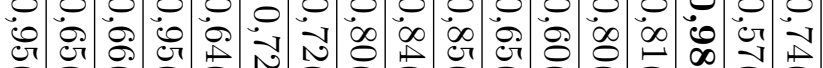

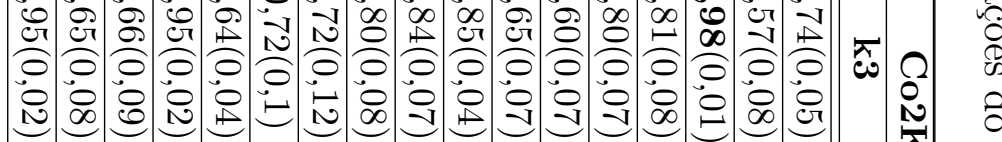

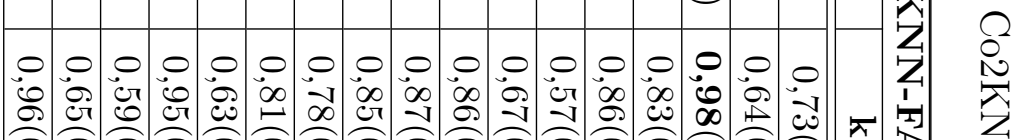

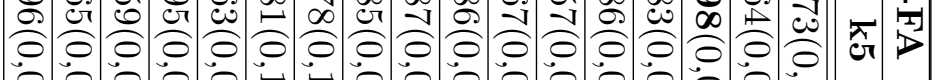

定

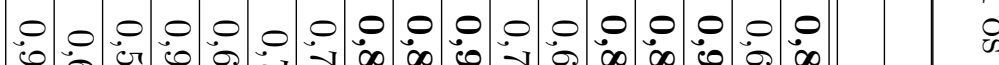

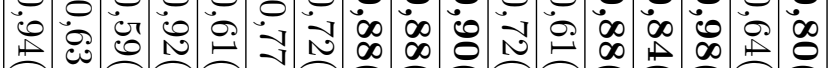

苛

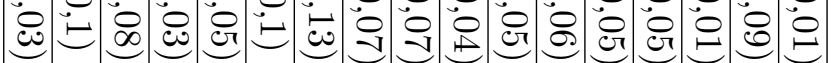

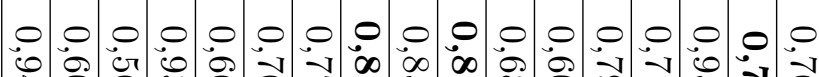

占

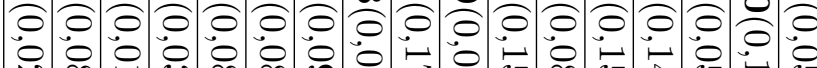

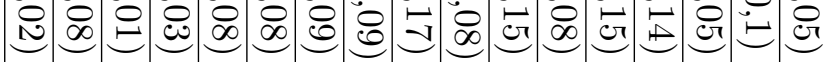

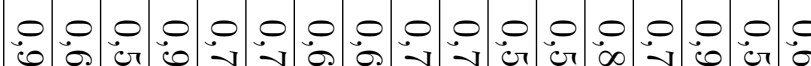

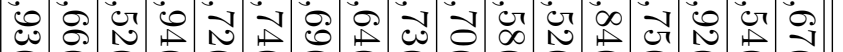

○)

昰

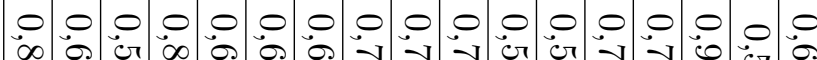

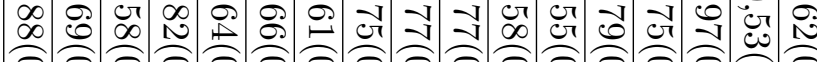

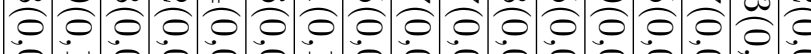

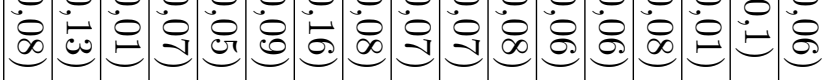

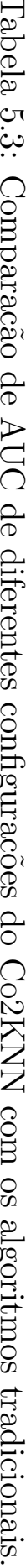


Tabela 5.4: Co2KNN vs Algoritmos tradicionais com teste estatístico.

\begin{tabular}{|c|c|c|c|c|}
\hline \multirow{2}{*}{ Classifier } & \multirow{2}{*}{$\mathbf{k}$} & \multicolumn{3}{|c|}{ Selftraining } \\
\cline { 2 - 5 } & & Bayes & J48 & SVM \\
\hline \hline \multirow{4}{*}{ Co2KNN } & 1 & não & não & não \\
\cline { 2 - 5 } & 3 & não & sim & sim \\
\cline { 2 - 5 } & 5 & sim & sim & sim \\
\cline { 2 - 5 } & 15 & sim & sim & sim \\
\hline \multirow{4}{*}{ Co2KNN-FA } & 1 & não & não & não \\
\cline { 2 - 5 } & 3 & não & não & não \\
\cline { 2 - 5 } & 5 & não & não & sim \\
\cline { 2 - 5 } & 15 & não & sim & sim \\
\hline
\end{tabular}

Um detalhe importante a ser considerado é que o valor de $k$ é um parâmetro relevante no desempenho da classificação. Portanto, para cada problema de classificação, é necessário analisar qual valor de $k$ é o apropriado, esperando-se, dessa forma, um desempenho ainda melhor do que quando comparado com o valor de $k$ fixo. Também pode-se notar que o desempenho do classificador Co2KNN é melhor do que o Co2KNN-FA. Uma análise mais detalhada de tal comparação é feita na Seção 5.4.5.

\subsubsection{Comparação com as Estratégias Isoladas}

Com o objetivo de conferir se, de fato, a combinação das estratégias KNN Local e Global permite uma melhora na classificação, o classificador Co2KNN foi comparado com as versões semi-supervisionadas do KNN Local e Global separadamente. Para tal tarefa, foram configuradas diferentes versões do algoritmo Selftraining com cada uma das estratégias e valores de $k \in\{1,3,5,15\}$. Os resultados da comparação são apresentados nas Tabelas 5.5, 5.6, 5.7 e 5.8. Como pode ser observado nos valores ressaltados, o algoritmo Co2KNN obteve um melhor desempenho na maioria das configurações dos experimentos. Ressalta-se que cada comparação foi feita separadamente pois o valor do parâmetro $k$ é dado de entrada tanto para o algoritmo Co2KNN quanto para as estratégias KNN Local e Global treinadas com Selftraining.

É interessante mencionar que na maioria dos casos o classificador Co2KNN-FA tem um desempenho melhor do que as estratégias separadas, porém, em poucos casos supera o classificador Co2KNN.

Para mostrar estatisticamente a superioridade do classificador Co2KNN, foi aplicado o teste de Friedman em conjunto com o método de Dunn, conforme mostrado na Tabela 5.9. Note-se que o classificador Co $2 \mathrm{KNN}$ obteve uma diferença significativa na maioria das configurações, mostrando que o algoritmo Co2KNN consegue realizar um aprendizado de ajuda conjunta entre os classificadores corrigindo os possíveis erros que algum classificador possa ter com base nas confianças preditas pelo outro classificador, o que mostra que a combinação das duas estratégias com o algoritmo Co2KNN obtêm melhores resultados do que quando utilizada cada estratégia separadamente. É importante observar que, para valores de $k \in\{3,5\}$, o classificador Co2KNN não obteve uma diferença significativa com 
Tabela 5.5: Comparação de AUC do Co2KNN com as estratégias separadas para $k=1$.

\begin{tabular}{|c|c|c|c|c|}
\hline \multirow{2}{*}{ Nome } & Co2KNN & Co2KNN-FA & \multicolumn{2}{|c|}{ Selftraining } \\
\cline { 2 - 5 } & $\mathbf{k 1}$ & $\mathbf{k 1}$ & KNN Local & KNN Global \\
\hline \hline adult & $\mathbf{0 , 6 7}(0,04)$ & $\mathbf{0 , 6 7}(0,04)$ & $0,58(0,04)$ & $0,54(0,02)$ \\
\hline breast-c & $\mathbf{0 , 6 3}(0,08)$ & $0,61(0,09)$ & $0,58(0,15)$ & $0,53(0,11)$ \\
\hline breast-w & $\mathbf{0 , 9 7}(0,01)$ & $\mathbf{0 , 9 7}(0,01)$ & $\mathbf{0 , 9 7}(0,01)$ & $0,81(0,1)$ \\
\hline colic & $\mathbf{0 , 7 3}(0,05)$ & $\mathbf{0 , 7 3}(0,07)$ & $\mathbf{0 , 7 2}(0,12)$ & $0,53(0,08)$ \\
\hline credit-a & $\mathbf{0 , 7 6}(0,04)$ & $0,71(0,05)$ & $0,67(0,08)$ & $0,57(0,03)$ \\
\hline credit-g & $\mathbf{0 , 5 8}(0,05)$ & $0,54(0,06)$ & $\mathbf{0 , 5 7}(0,04)$ & $0,52(0,04)$ \\
\hline diabetes & $\mathbf{0 , 6 1}(0,03)$ & $0,59(0,06)$ & $\mathbf{0 , 6 1}(0,07)$ & $0,56(0,04)$ \\
\hline heart-c & $\mathbf{0 , 7 7}(0,07)$ & $0,73(0,08)$ & $0,73(0,11)$ & $0,61(0,09)$ \\
\hline heart-h & $\mathbf{0 , 7 5}(0,07)$ & $\mathbf{0 , 7 6}(0,09)$ & $0,74(0,17)$ & $0,61(0,06)$ \\
\hline heart-s & $\mathbf{0 , 7 1}(0,07)$ & $\mathbf{0 , 7 1}(0,08)$ & $\mathbf{0 , 7 1}(0,09)$ & $0,53(0,07)$ \\
\hline hepatitis & $0,65(0,08)$ & $0,61(0,11)$ & $\mathbf{0 , 6 7}(0,09)$ & $0,56(0,09)$ \\
\hline ionosphere & $\mathbf{0 , 7 9}(0,07)$ & $0,70(0,04)$ & $0,74(0,12)$ & $0,57(0,07)$ \\
\hline kr-vs-kp & $\mathbf{0 , 7 8}(0,03)$ & $0,64(0,06)$ & $0,66(0,03)$ & $0,64(0,03)$ \\
\hline mushroom & $\mathbf{0 , 9 6}(0,01)$ & $0,94(0,02)$ & $0,94(0,02)$ & $0,73(0,06)$ \\
\hline sick & $\mathbf{0 , 6 2}(0,07)$ & $0,56(0,06)$ & $0,58(0,06)$ & $0,57(0,02)$ \\
\hline sonar & $\mathbf{0 , 7 2}(0,07)$ & $0,70(0,08)$ & $0,65(0,1)$ & $0,66(0,07)$ \\
\hline vote & $\mathbf{0 , 9 4}(0,05)$ & $\mathbf{0 , 9 3}(0,04)$ & $0,92(0,05)$ & $0,78(0,07)$ \\
\hline
\end{tabular}

Tabela 5.6: Comparação de AUC do Co2KNN com as estratégias separadas para $k=3$.

\begin{tabular}{|c|c|c|c|c|}
\hline \multirow{2}{*}{ Nome } & Co2KNN & Co2KNN-FA & \multicolumn{2}{|c|}{ Selftraining } \\
\cline { 2 - 5 } & k3 & k3 & KNN Local & KNN Global \\
\hline \hline adult & $\mathbf{0 , 7 7}(0,02)$ & $0,74(0,05)$ & $0,71(0,04)$ & $0,59(0,05)$ \\
\hline breast-c & $\mathbf{0 , 6 6}(0,09)$ & $0,57(0,08)$ & $\mathbf{0 , 6 7}(0,1)$ & $0,64(0,17)$ \\
\hline breast-w & $\mathbf{0 , 9 8}(0,01)$ & $\mathbf{0 , 9 8}(0,01)$ & $\mathbf{0 , 9 8}(0,01)$ & $0,90(0,06)$ \\
\hline colic & $\mathbf{0 , 8 3}(0,04)$ & $0,81(0,08)$ & $0,80(0,12)$ & $0,70(0,06)$ \\
\hline credit-a & $0,85(0,04)$ & $0,80(0,07)$ & $\mathbf{0 , 8 7}(0,08)$ & $0,71(0,09)$ \\
\hline credit-g & $\mathbf{0 , 6 4}(0,05)$ & $0,60(0,07)$ & $0,61(0,07)$ & $0,56(0,03)$ \\
\hline diabetes & $\mathbf{0 , 7 0}(0,06)$ & $0,65(0,07)$ & $0,63(0,05)$ & $0,57(0,06)$ \\
\hline heart-c & $\mathbf{0 , 8 4}(0,06)$ & $\mathbf{0 , 8 5}(0,04)$ & $0,83(0,09)$ & $0,67(0,07)$ \\
\hline heart-h & $\mathbf{0 , 8 5}(0,05)$ & $\mathbf{0 , 8 4}(0,07)$ & $\mathbf{0 , 8 5}(0,09)$ & $0,72(0,12)$ \\
\hline heart-s & $\mathbf{0 , 8 3}(0,03)$ & $0,80(0,08)$ & $\mathbf{0 , 8 2}(0,1)$ & $0,69(0,09)$ \\
\hline hepatitis & $\mathbf{0 , 7 9}(0,1)$ & $0,72(0,12)$ & $0,75(0,15)$ & $0,70(0,15)$ \\
\hline ionosphere & $\mathbf{0 , 8 6}(0,05)$ & $0,72(0,1)$ & $0,74(0,15)$ & $0,59(0,09)$ \\
\hline kr-vs-kp & $\mathbf{0 , 8 2}(0,05)$ & $0,64(0,04)$ & $0,70(0,05)$ & $0,69(0,03)$ \\
\hline mushroom & $\mathbf{0 , 9 7}(0,01)$ & $0,95(0,02)$ & $0,94(0,02)$ & $0,87(0,04)$ \\
\hline sick & $\mathbf{0 , 6 5}(0,06)$ & $\mathbf{0 , 6 6}(0,09)$ & $0,60(0,06)$ & $0,61(0,07)$ \\
\hline sonar & $\mathbf{0 , 7 7}(0,1)$ & $0,65(0,08)$ & $0,72(0,13)$ & $0,68(0,12)$ \\
\hline vote & $\mathbf{0 , 9 4}(0,01)$ & $\mathbf{0 , 9 5}(0,02)$ & $\mathbf{0 , 9 5}(0,04)$ & $0,88(0,06)$ \\
\hline
\end{tabular}

o classificador semi-supervisionado que utiliza a estratégia do KNN Local. Observando-se os resultados isolados do KNN Local e Global para diferentes $k$, nota-se que o KNN Global tem desempenho muio fraco para os menores valores de $k$. Portanto, é de se esperar que, para valores acima de 5, a contribuição do KNN Global passe ser mais efetiva para o desempenho final da abordagem Co2KNN. Uma análise mais detalhada das estratégias Local e Global é apresentada na próxima seção. Já com o classificador Co2KNN-FA, 
Tabela 5.7: Comparação de AUC do Co2KNN com as estratégias separadas para $k=5$.

\begin{tabular}{|c|c|c|c|c|}
\hline \multirow{2}{*}{ Nome } & Co2KNN & Co2KNN-FA & \multicolumn{2}{|c|}{ Selftraining } \\
\cline { 2 - 5 } & $\mathbf{k 5}$ & $\mathbf{k 5}$ & KNN Local & KNN Global \\
\hline \hline adult & $\mathbf{0 , 7 7}(0,02)$ & $0,73(0,1)$ & $0,69(0,05)$ & $0,66(0,06)$ \\
\hline breast-c & $\mathbf{0 , 6 4}(0,1)$ & $\mathbf{0 , 6 4}(0,08)$ & $\mathbf{0 , 6 5}(0,11)$ & $0,59(0,12)$ \\
\hline breast-w & $\mathbf{0 , 9 9}(0,01)$ & $\mathbf{0 , 9 8}(0,01)$ & $\mathbf{0 , 9 8}(0,01)$ & $0,91(0,04)$ \\
\hline colic & $\mathbf{0 , 8 5}(0,07)$ & $0,83(0,06)$ & $0,82(0,11)$ & $0,71(0,12)$ \\
\hline credit-a & $\mathbf{0 , 8 6}(0,02)$ & $\mathbf{0 , 8 6}(0,04)$ & $0,83(0,05)$ & $0,79(0,06)$ \\
\hline credit-g & $\mathbf{0 , 6 4}(0,06)$ & $0,57(0,09)$ & $0,62(0,09)$ & $0,57(0,07)$ \\
\hline diabetes & $\mathbf{0 , 7 3}(0,06)$ & $0,67(0,07)$ & $0,68(0,06)$ & $0,61(0,06)$ \\
\hline heart-c & $\mathbf{0 , 8 6}(0,05)$ & $\mathbf{0 , 8 6}(0,07)$ & $\mathbf{0 , 8 7}(0,06)$ & $0,72(0,09)$ \\
\hline heart-h & $\mathbf{0 , 8 6}(0,05)$ & $\mathbf{0 , 8 7}(0,08)$ & $\mathbf{0 , 8 6}(0,12)$ & $0,73(0,12)$ \\
\hline heart-s & $\mathbf{0 , 8 6}(0,09)$ & $\mathbf{0 , 8 5}(0,04)$ & $0,82(0,08)$ & $0,77(0,09)$ \\
\hline hepatitis & $\mathbf{0 , 7 7}(0,15)$ & $\mathbf{0 , 7 8}(0,12)$ & $0,71(0,12)$ & $0,72(0,17)$ \\
\hline ionosphere & $\mathbf{0 , 8 6}(0,05)$ & $0,81(0,11)$ & $0,78(0,13)$ & $0,70(0,08)$ \\
\hline kr-vs-kp & $\mathbf{0 , 8 3}(0,02)$ & $0,63(0,08)$ & $0,68(0,06)$ & $0,72(0,03)$ \\
\hline mushroom & $\mathbf{0 , 9 6}(0,01)$ & $\mathbf{0 , 9 5}(0,01)$ & $0,92(0,01)$ & $0,89(0,02)$ \\
\hline sick & $\mathbf{0 , 7 0}(0,09)$ & $0,59(0,09)$ & $0,61(0,05)$ & $0,65(0,11)$ \\
\hline sonar & $\mathbf{0 , 7 6}(0,1)$ & $0,65(0,09)$ & $0,69(0,11)$ & $0,71(0,13)$ \\
\hline vote & $\mathbf{0 , 9 8}(0,02)$ & $0,96(0,02)$ & $0,93(0,04)$ & $0,88(0,06)$ \\
\hline
\end{tabular}

Tabela 5.8: Comparação de AUC do Co2KNN com as estratégias separadas para $k=15$.

\begin{tabular}{|c|c|c|c|c|}
\hline \multirow{2}{*}{ Nome } & Co2KNN & Co2KNN-FA & \multicolumn{2}{|c|}{ Selftraining } \\
\cline { 2 - 5 } & k15 & k15 & KNN Local & KNN Global \\
\hline \hline adult & $\mathbf{0 , 8 0}(0,02)$ & $\mathbf{0 , 8 0}(0,01)$ & $0,63(0,06)$ & $0,78(0,04)$ \\
\hline breast-c & $\mathbf{0 , 7 0}(0,09)$ & $0,64(0,09)$ & $0,6(0,18)$ & $0,63(0,12)$ \\
\hline breast-w & $\mathbf{0 , 9 8}(0,01)$ & $\mathbf{0 , 9 8}(0,01)$ & $\mathbf{0 , 9 9}(0,01)$ & $0,94(0,05)$ \\
\hline colic & $\mathbf{0 , 8 5}(0,07)$ & $\mathbf{0 , 8 4}(0,05)$ & $0,80(0,09)$ & $0,78(0,16)$ \\
\hline credit-a & $\mathbf{0 , 8 9}(0,03)$ & $\mathbf{0 , 8 8}(0,05)$ & $0,83(0,04)$ & $0,87(0,03)$ \\
\hline credit-g & $\mathbf{0 , 6 6}(0,04)$ & $0,61(0,06)$ & $0,63(0,06)$ & $0,63(0,07)$ \\
\hline diabetes & $\mathbf{0 , 7 5}(0,03)$ & $0,72(0,05)$ & $0,64(0,11)$ & $0,69(0,08)$ \\
\hline heart-c & $\mathbf{0 , 9 0}(0,04)$ & $\mathbf{0 , 9 0}(0,04)$ & $0,87(0,06)$ & $0,84(0,05)$ \\
\hline heart-h & $\mathbf{0 , 8 9}(0,05)$ & $\mathbf{0 , 8 8}(0,07)$ & $0,86(0,08)$ & $0,87(0,08)$ \\
\hline heart-s & $\mathbf{0 , 8 8}(0,05)$ & $\mathbf{0 , 8 8}(0,07)$ & $0,84(0,12)$ & $0,86(0,05)$ \\
\hline hepatitis & $\mathbf{0 , 8 0}(0,09)$ & $0,72(0,13)$ & $\mathbf{0 , 8 1}(0,12)$ & $0,76(0,22)$ \\
\hline ionosphere & $\mathbf{0 , 8 1}(0,09)$ & $0,77(0,1)$ & $0,58(0,1)$ & $0,58(0,1)$ \\
\hline kr-vs-kp & $\mathbf{0 , 7 9}(0,04)$ & $0,61(0,05)$ & $0,69(0,1)$ & $0,71(0,07)$ \\
\hline mushroom & $\mathbf{0 , 9 6}(0,01)$ & $0,92(0,03)$ & $0,90(0,02)$ & $0,88(0,03)$ \\
\hline sick & $\mathbf{0 , 6 7}(0,09)$ & $0,59(0,08)$ & $0,64(0,08)$ & $0,61(0,09)$ \\
\hline sonar & $\mathbf{0 , 7 1}(0,07)$ & $0,63(0,1)$ & $0,63(0,1)$ & $0,62(0,12)$ \\
\hline vote & $\mathbf{0 , 9 7}(0,02)$ & $0,94(0,03)$ & $\mathbf{0 , 9 6}(0,03)$ & $\mathbf{0 , 9 7}(0,06)$ \\
\hline
\end{tabular}

não se obteve uma diferença estatisticamente significativa com o classificador baseado na estratégia KNN Local.

\subsubsection{Comparação entre o KNN Local e o KNN Global}

Adicionalmente, os resultados mostrados nos experimentos anteriores podem ser utilizados para analisar a estratégia KNN Global separadamente e o classificador baseado 
Tabela 5.9: Co2KNN vs Selftraining com teste estatístico

\begin{tabular}{|c|c|c|c|}
\hline \multirow{2}{*}{ Classifier } & \multirow{2}{*}{$\mathbf{k}$} & \multicolumn{2}{|c|}{ Selftraining } \\
\cline { 2 - 4 } & & Local KNN & Global KNN \\
\hline \hline \multirow{3}{*}{ Co2KNN } & 1 & não & sim \\
\cline { 2 - 4 } & 3 & não & sim \\
\cline { 2 - 4 } & 5 & $\operatorname{sim}$ & $\operatorname{sim}$ \\
\cline { 2 - 4 } & 15 & $\operatorname{sim}$ & $\operatorname{sim}$ \\
\hline \multirow{4}{*}{ Co2KNN-FA } & 1 & não & sim \\
\cline { 2 - 4 } & 3 & não & sim \\
\cline { 2 - 4 } & 5 & não & sim \\
\cline { 2 - 4 } & 15 & não & não \\
\hline
\end{tabular}

nesta estratégia.

Visando analisar com maior detalhe as diferenças entre as estratégias KNN Global e KNN Local, foram comparados os diferentes tamanhos dos conjuntos de vizinhos obtidos pela KNN Global, visando analisar de uma forma quantitativa a diferença da geração dos conjuntos a partir das estratégias KNN Local e KNN Global. As comparações para cada base são mostradas na Figura 5.3, sendo que a quantidade de vizinhos recuperados pela estratégia KNN Global está representada pelas barras verticais e a quantidade de vizinhos obtida pela KNN Local é representada por uma linha horizontal pontilhada devido ao fato que é constante e igual a $k$. É visível que na grande maioria dos casos, a tendência do KNN Global é gerar conjuntos menores do que o KNN Local. Este fato mostrou que no cenário mais comum, os conjuntos mostram uma distribuição dos dados mais compacta dentro das classes, diminuindo a possibilidade de gerar arestas de entrada ao novo exemplo. Entretanto, nos poucos casos nos quais o KNN Global gera conjunto maiores, pode-se afirmar que a distribuição dos dados é mais esparsa, favorecendo a aparição de arestas incidentes nos novos exemplos. Entre as bases que mostram essa distribuição mais esparsa no processo de treinamento do Co2KNN para $k=3$ estão a breast-c, $k r$-vs-kp e a vote. Ressalta-se que, quanto maior for o valor de $k$, o KNN Global tende a gerar conjuntos maiores, sendo este um possível motivo da melhora do classificador baseado no KNN Global e, consequentemente, do algoritmo Co2KNN com valores maiores de $k$.

Adicionalmente, após a aplicação do teste estatístico de Wilcoxon, pode-se observar que o KNN Local consegue um desempenho significativamente melhor do que o KNN Global em todos os cenários exceto quando $k=15$, como mostrado na Tabela 5.10.

Tabela 5.10: KNN Local vs KNN Global com teste estatístico.

\begin{tabular}{|c|c|c|c|c|c|}
\hline \multirow{2}{*}{ Classifier } & \multirow{2}{*}{$\mathbf{k}$} & \multicolumn{5}{|c|}{ KNN Global } \\
\cline { 2 - 6 } & & 1 & 3 & 5 & 15 \\
\hline \hline \multirow{4}{*}{ KNN Local } & 1 & sim & - & - & - \\
\cline { 2 - 6 } & 3 & - & sim & - & - \\
\cline { 2 - 6 } & 5 & - & - & sim & - \\
\cline { 2 - 6 } & 15 & - & - & - & não \\
\hline
\end{tabular}

Com os resultados do teste estatístico e a análise feita em relação à quantidade de 


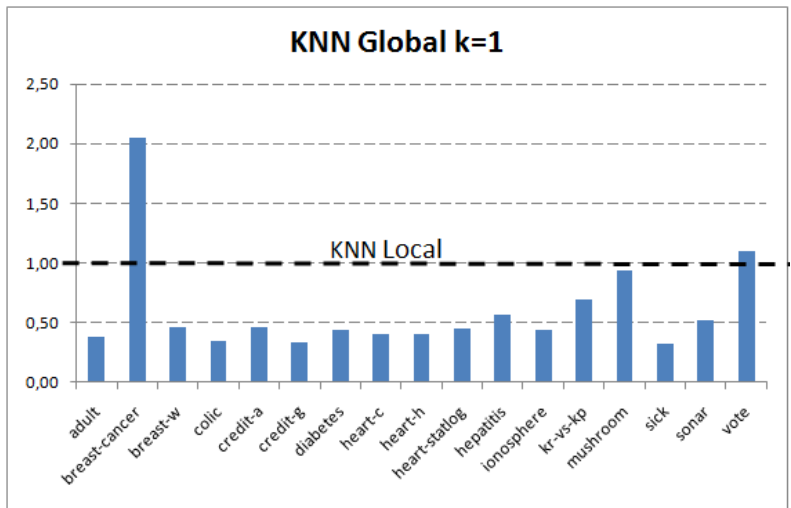

(a)

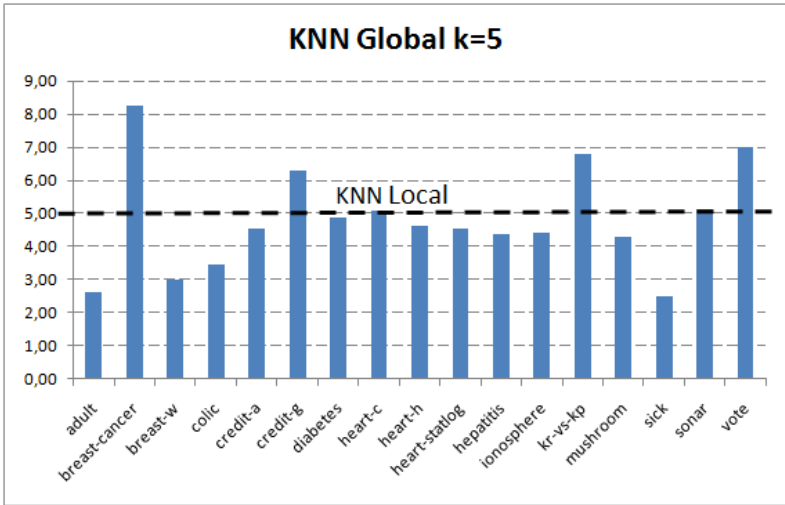

(c)

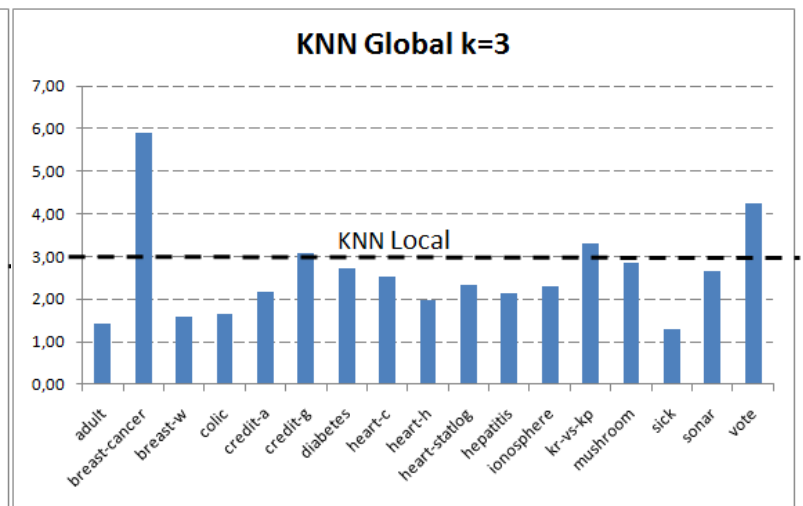

(b)

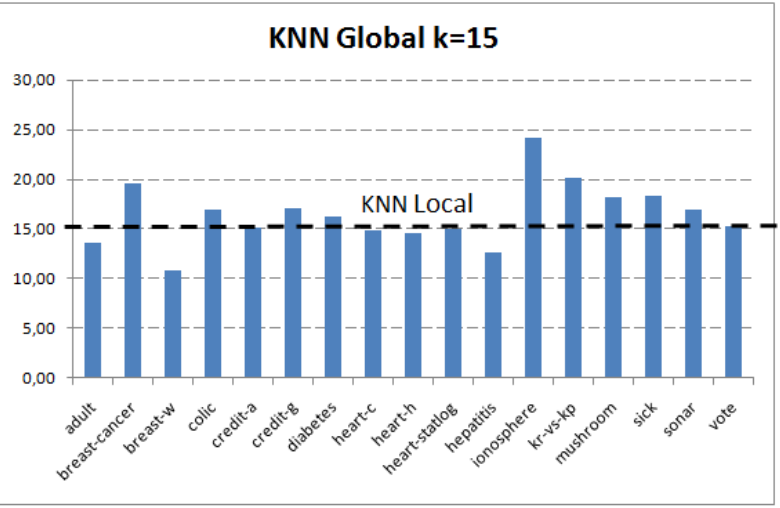

(d)

Figura 5.3: Comparação entre o número fixo de vizinhos recuperados pelo KNN Local e o número variável recuperado pelo KNN Global para $k \in[1,3,5,15]$.

vizinhos recuperados pela estratégia KNN Global, pode-se concluir que a medida que o valor de $k$ aumenta, o classificador baseado na estratégia KNN Global melhora mais seu desempenho e, por sua vez, o desempenho do classificador Co2KNN. Na Figura 5.4 é ilustrada a comparação entre a melhora de cada estratégia em relação ao valor de $k$. São mostradas unicamente 4 bases para uma rápida comparação visual, porém, todas as figuras desta comparação estão colocadas no Apêndice A. Ressalta-se que, para a maioria das demais bases de dados utilizadas, o KNN Global consegue melhorar bastante o seu desempenho com $k=15$. Somente para as bases ionosphere, sick e sonar o processo de aprendizado da estratégia KNN Global sofre com valor alto de $k$, como mostrado na Figura 5.4c. Em termos gerais, pode-se concluir que a melhora do desempenho do KNN Global é maior do que a melhora do KNN Local, sendo esta um dos motivos mais importantes para o sucesso do algoritmo Co2KNN.

\subsubsection{Comparação com o SKNN}

Adicionalmente, o algoritmo Co2KNN também foi comparado com o SKNN Nock et al. (2000). O classificador SKNN também foi treinado com o algoritmo semi-supervisionado Selftraining e com os mesmos parâmetros do algoritmo Co2KNN. É importante consid- 


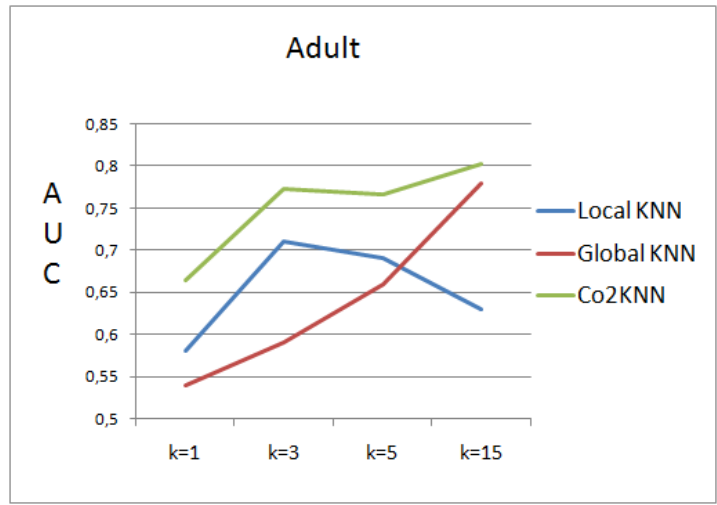

(a)

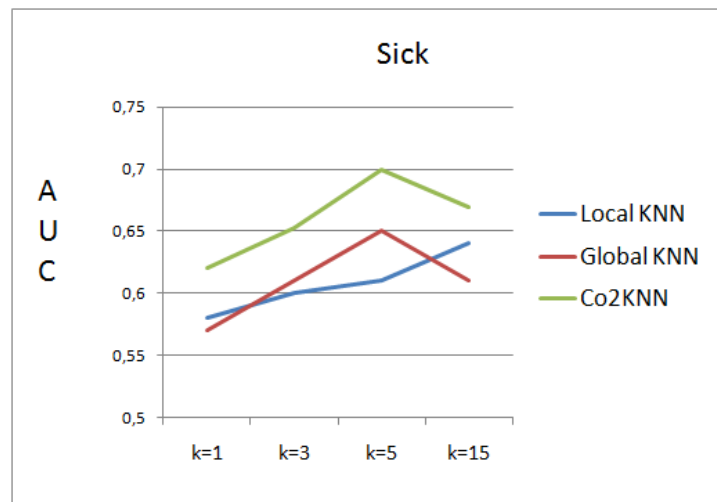

(c)

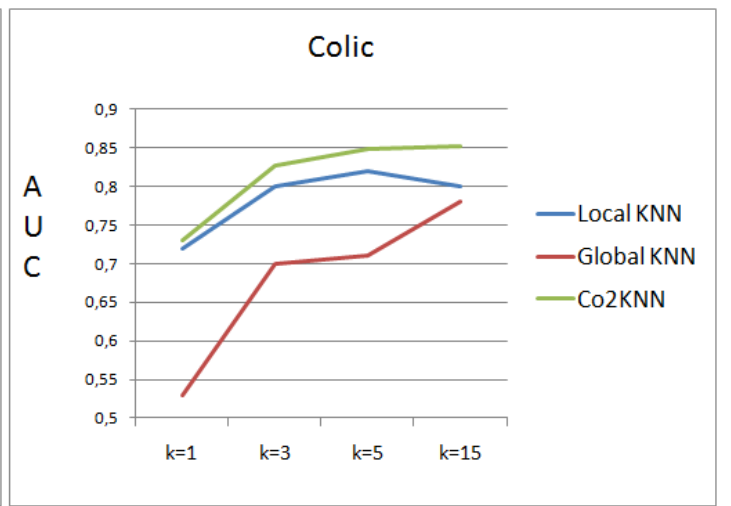

(b)

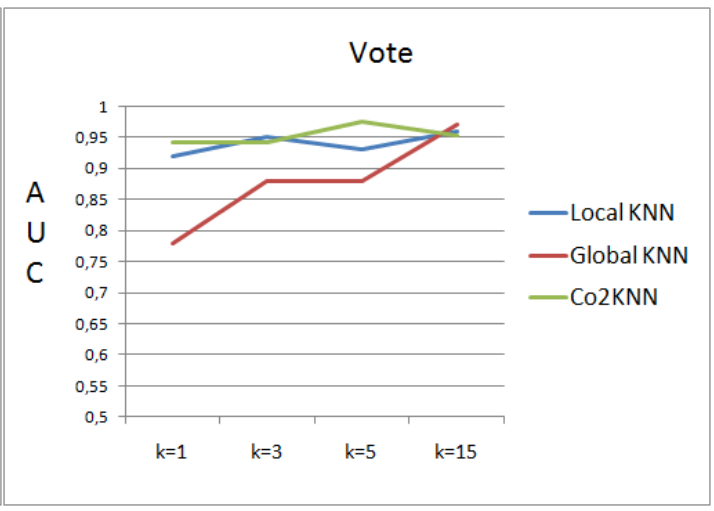

(d)

Figura 5.4: Comparação de AUC entre o desempenho em relação ao crescimento de $K$ em 4 bases.

erar que o SKNN foi alterado utilizando a mesma estratégia proposta para recuperar os vizinhos com o KNN Global. Com isso, a versão semi-supervisionado do algoritmo SKNN apresenta uma complexidade computacional igual do que o algoritmo Co2KNN. O resumo dos resultados medidos com o AUC são mostrados nas Tabelas 5.11 , 5.12, 5.13 e 5.14, para valores de $k \in\{1,3,5,15\}$.

Os resultados mostram-se bem similares, sendo que o SKNN consegue obter um resultado muito bom com $k=1$. Contudo, com valores $k$ maiores do que 1 , o classificador Co2KNN mostrou-se mais robusto, obtendo um melhor desempenho na maior parte dos cenários.

Como mostrado na Tabela 5.15, aplicou-se o teste estatístico de Wilcoxon para a comparação dos classificadores Co2KNN e Co2KNN-FA com o SKNN. Pode-se observar que os resultados dos classificadores SKNN e o Co2KNN não são significativamente diferentes quando utilizado para $k=1$, enquanto que o SKNN consegue uma diferença significativa em relação ao Co2KNN-FA. Nota-se que o classificador Co2KNN consegue obter diferença significativa em relação ao SKNN para $k>1$. Uma hipótese é que com o valor de $k=1$ o classificador Co2KNN não consegue aproveitar a separação dos conjuntos para a inferência do rótulo, provavelmente causado pela alta quantidade de conjuntos vazios que a estratégia KNN Global gera. A quantidade de conjuntos vazios gerados pelo classificador 
Tabela 5.11: Comparação de AUC do Co2KNN com o SKNN para valor de $k=1$.

\begin{tabular}{|c|c|c|c|}
\hline \multirow{2}{*}{ Nome } & Co2KNN & Co2KNN-FA & Selftraining \\
\cline { 2 - 4 } & $\mathbf{k 1}$ & $\mathbf{k 1}$ & SKNN \\
\hline \hline adult & $0,67(0,04)$ & $0,67(0,04)$ & $\mathbf{0 , 7 2}(0,04)$ \\
\hline breast-c & $\mathbf{0 , 6 3}(0,08)$ & $0,61(0,09)$ & $\mathbf{0 , 6 3}(0,2)$ \\
\hline breast-w & $\mathbf{0 , 9 7}(0,01)$ & $\mathbf{0 , 9 7}(0,01)$ & $\mathbf{0 , 9 7}(0,01)$ \\
\hline colic & $0,73(0,05)$ & $0,73(0,07)$ & $\mathbf{0 , 7 6}(0,11)$ \\
\hline credit-a & $0,76(0,04)$ & $0,71(0,05)$ & $\mathbf{0 , 8 3}(0,05)$ \\
\hline credit-g & $\mathbf{0 , 5 8}(0,05)$ & $0,54(0,06)$ & $0,56(0,06)$ \\
\hline diabetes & $0,61(0,03)$ & $0,59(0,06)$ & $\mathbf{0 , 6 5}(0,06)$ \\
\hline heart-c & $0,77(0,07)$ & $0,73(0,08)$ & $\mathbf{0 , 8 4}(0,09)$ \\
\hline heart-h & $0,75(0,07)$ & $0,76(0,09)$ & $\mathbf{0 , 7 9}(0,14)$ \\
\hline heart-s & $0,71(0,07)$ & $0,71(0,08)$ & $\mathbf{0 , 7 7}(0,13)$ \\
\hline hepatitis & $0,65(0,08)$ & $0,61(0,11)$ & $\mathbf{0 , 7 4}(0,12)$ \\
\hline ionosphere & $\mathbf{0 , 7 9}(0,07)$ & $0,70(0,04)$ & $0,67(0,08)$ \\
\hline kr-vs-kp & $\mathbf{0 , 7 8}(0,03)$ & $0,64(0,06)$ & $0,66(0,04)$ \\
\hline mushroom & $\mathbf{0 , 9 6}(0,01)$ & $0,94(0,02)$ & $0,94(0,02)$ \\
\hline sick & $0,62(0,07)$ & $0,56(0,06)$ & $\mathbf{0 , 6 4}(0,12)$ \\
\hline sonar & $\mathbf{0 , 7 2}(0,07)$ & $0,70(0,08)$ & $\mathbf{0 , 7 1}(0,14)$ \\
\hline vote & $\mathbf{0 , 9 4}(0,05)$ & $0,93(0,04)$ & $\mathbf{0 , 9 5}(0,04)$ \\
\hline
\end{tabular}

Tabela 5.12: Comparação de AUC do Co2KNN com o SKNN para valor de $k=3$.

\begin{tabular}{|c|c|c|c|}
\hline \multirow{2}{*}{ Nome } & Co2KNN & Co2KNN-FA & Selftraining \\
\cline { 2 - 4 } & $\mathbf{k 3}$ & $\mathbf{k 3}$ & SKNN \\
\hline \hline adult & $0,77(0,02)$ & $0,74(0,05)$ & $0,78(0,02)$ \\
\hline breast-c & $0,66(0,09)$ & $0,57(0,08)$ & $\mathbf{0 , 6 8}(0,21)$ \\
\hline breast-w & $\mathbf{0 , 9 8}(0,01)$ & $\mathbf{0 , 9 8}(0,01)$ & $\mathbf{0 , 9 7}(0,02)$ \\
\hline colic & $\mathrm{k}(0,04)$ & $0,81(0,08)$ & $0,75(0,08)$ \\
\hline credit-a & $\mathbf{0 , 8 5}(0,04)$ & $0,80(0,07)$ & $\mathrm{k}(0,04)$ \\
\hline credit-g & $\mathbf{0 , 6 4}(0,05)$ & $0,60(0,07)$ & $0,61(0,05)$ \\
\hline diabetes & $\mathbf{0 , 7 0}(0,06)$ & $0,65(0,07)$ & $0,64(0,06)$ \\
\hline heart-c & $0,84(0,06)$ & $\mathbf{0 , 8 5}(0,04)$ & $\mathbf{0 , 8 6}(0,06)$ \\
\hline heart-h & $\mathbf{0 , 8 5}(0,05)$ & $\mathbf{0 , 8 4}(0,07)$ & $\mathbf{0 , 8 4}(0,13)$ \\
\hline heart-s & $\mathbf{0 , 8 3}(0,03)$ & $0,80(0,08)$ & $0,79(0,08)$ \\
\hline hepatitis & $\mathbf{0 , 7 9}(0,1)$ & $0,72(0,12)$ & $0,73(0,12)$ \\
\hline ionosphere & $\mathbf{0 , 8 6}(0,05)$ & $0,72(0,1)$ & $0,68(0,15)$ \\
\hline kr-vs-kp & $\mathbf{0 , 8 2}(0,05)$ & $0,64(0,04)$ & $0,67(0,04)$ \\
\hline mushroom & $\mathbf{0 , 9 7}(0,01)$ & $0,95(0,02)$ & $0,93(0,02)$ \\
\hline sick & $\mathbf{0 , 6 5}(0,06)$ & $\mathbf{0 , 6 6}(0,09)$ & $\mathbf{0 , 6 5}(0,16)$ \\
\hline sonar & $\mathbf{0 , 7 7}(0,1)$ & $0,65(0,08)$ & $0,73(0,1)$ \\
\hline vote & $\mathbf{0 , 9 4}(0,01)$ & $\mathbf{0 , 9 5}(0,02)$ & $\mathbf{0 , 9 5}(0,02)$ \\
\hline
\end{tabular}

KNN Global pode ser observado na Figura 5.3a. Nessa Figura é mostrada que a média da quantidade de vizinhos obtida pela estratégia KNN Global para $k=1$ é menor a 1 na maioria dos casos. Como a quantidade de vizinhos é uma variável discreta e inteira, é claro que em uma parte considerável das iterações gerou conjuntos vazios. Analisando o desempenho do classificador Co2KNN-FA, somente conseguiu obter diferença significativa do SKNN para o valor de $k=15$, tendo um desempenho estatisticamente igual ao 
Tabela 5.13: Comparação de AUC do Co2KNN com o SKNN para valor de $k=5$.

\begin{tabular}{|c|c|c|c|}
\hline \multirow{2}{*}{ Nome } & Co2KNN & Co2KNN-FA & Selftraining \\
\cline { 2 - 4 } & k5 & k5 & SKNN \\
\hline \hline adult & $\mathbf{0 , 7 7}(0,02)$ & $0,73(0,1)$ & $\mathbf{0 , 7 7}(0,03)$ \\
\hline breast-c & $\mathbf{0 , 6 9}(0,1)$ & $0,64(0,08)$ & $0,66(0,13)$ \\
\hline breast-w & $\mathbf{0 , 9 9}(0,01)$ & $\mathbf{0 , 9 8}(0,01)$ & $\mathbf{0 , 9 8}(0,02)$ \\
\hline colic & $\mathbf{0 , 8 5}(0,07)$ & $0,83(0,06)$ & $0,83(0,07)$ \\
\hline credit-a & $\mathbf{0 , 8 6}(0,02)$ & $\mathbf{0 , 8 6}(0,04)$ & $\mathbf{0 , 8 5}(0,06)$ \\
\hline credit-g & $0,64(0,06)$ & $\mathbf{0 , 6 7}(0,09)$ & $0,64(0,06)$ \\
\hline diabetes & $\mathbf{0 , 7 3}(0,06)$ & $0,67(0,07)$ & $0,70(0,05)$ \\
\hline heart-c & $\mathbf{0 , 8 6}(0,05)$ & $\mathbf{0 , 8 6}(0,07)$ & $\mathbf{0 , 8 5}(0,1)$ \\
\hline heart-h & $\mathbf{0 , 8 6}(0,05)$ & $\mathbf{0 , 8 7}(0,08)$ & $0,83(0,07)$ \\
\hline heart-s & $\mathbf{0 , 8 6}(0,09)$ & $\mathbf{0 , 8 5}(0,04)$ & $0,84(0,09)$ \\
\hline hepatitis & $0,77(0,15)$ & $0,78(0,12)$ & $\mathbf{0 , 8 2}(0,26)$ \\
\hline ionosphere & $\mathbf{0 , 8 6}(0,05)$ & $0,81(0,11)$ & $0,73(0,13)$ \\
\hline kr-vs-kp & $\mathbf{0 , 8 3}(0,02)$ & $0,73(0,08)$ & $0,70(0,07)$ \\
\hline mushroom & $\mathbf{0 , 9 6}(0,01)$ & $\mathbf{0 , 9 5}(0,01)$ & $0,92(0,03)$ \\
\hline sick & $0,70(0,09)$ & $0,69(0,09)$ & $\mathbf{0 , 7 5}(0,07)$ \\
\hline sonar & $\mathbf{0 , 7 6}(0,1)$ & $0,65(0,09)$ & $0,64(0,12)$ \\
\hline vote & $\mathbf{0 , 9 8}(0,02)$ & $0,96(0,02)$ & $0,96(0,02)$ \\
\hline
\end{tabular}

Tabela 5.14: Comparação de AUC do Co2KNN com o SKNN para valor de $k=15$.

\begin{tabular}{|c|c|c|c|}
\hline \multirow{2}{*}{ Nome } & Co2KNN & Co2KNN-FA & Selftraining \\
\cline { 2 - 4 } & $\mathbf{k 1 5}$ & $\mathbf{k 1 5}$ & SKNN \\
\hline \hline adult & $\mathbf{0 , 8 0}(0,02)$ & $\mathbf{0 , 8 0}(0,01)$ & $0,70(0,12)$ \\
\hline breast-c & $\mathbf{0 , 7 0}(0,09)$ & $0,64(0,09)$ & $0,52(0,14)$ \\
\hline breast-w & $\mathbf{0 , 9 8}(0,01)$ & $\mathbf{0 , 9 8}(0,01)$ & $\mathbf{0 , 9 8}(0,02)$ \\
\hline colic & $\mathbf{0 , 8 5}(0,07)$ & $\mathbf{0 , 8 4}(0,05)$ & $0,80(0,1)$ \\
\hline credit-a & $\mathbf{0 , 8 9}(0,03)$ & $\mathbf{0 , 8 8}(0,05)$ & $0,86(0,11)$ \\
\hline credit-g & $\mathbf{0 , 6 6}(0,04)$ & $0,61(0,06)$ & $0,56(0,05)$ \\
\hline diabetes & $\mathbf{0 , 7 5}(0,03)$ & $0,72(0,05)$ & $0,66(0,11)$ \\
\hline heart-c & $\mathbf{0 , 9 0}(0,04)$ & $\mathbf{0 , 9 0}(0,04)$ & $0,88(0,06)$ \\
\hline heart-h & $\mathbf{0 , 8 9}(0,05)$ & $\mathbf{0 , 8 8}(0,07)$ & $\mathbf{0 , 8 8}(0,17)$ \\
\hline heart-s & $\mathbf{0 , 8 8}(0,05)$ & $\mathbf{0 , 8 8}(0,07)$ & $0,86(0,13)$ \\
\hline hepatitis & $0,80(0,09)$ & $\mathbf{0 , 8 5}(0,13)$ & $\mathbf{0 , 8 4}(0,21)$ \\
\hline ionosphere & $\mathbf{0 , 7 8}(0,09)$ & $\mathbf{0 , 7 7}(0,1)$ & $0,50(0,13)$ \\
\hline kr-vs-kp & $\mathbf{0 , 7 9}(0,04)$ & $0,61(0,05)$ & $0,69(0,06)$ \\
\hline mushroom & $\mathbf{0 , 9 6}(0,01)$ & $0,92(0,03)$ & $0,90(0,02)$ \\
\hline sick & $0,67(0,09)$ & $0,59(0,08)$ & $\mathbf{0 , 6 9}(0,07)$ \\
\hline sonar & $\mathbf{0 , 7 1}(0,07)$ & $0,63(0,1)$ & $0,60(0,08)$ \\
\hline vote & $0,95(0,02)$ & $0,94(0,03)$ & $\mathbf{0 , 9 7}(0,04)$ \\
\hline & & & \\
\hline
\end{tabular}

SKNN nos outros cenários, e perdendo para $k=1$. É interessante ver que para o caso de conjuntos recuperados vazios, o classificador baseado no algoritmo Global KNN teria uma confiança de 0.5 do exemplo pertencer a cada classificador, tendo um comportamento igual a um classificador aleatório. Esta é, provavelmente, o motivo do desempenho tão fraco do classificador Co2KNN e Co2KNN-FA quando $k=1$. Por tal motivo, não se recomenda o uso de $k=1$ 
Tabela 5.15: Co2KNN vs SKNN.

\begin{tabular}{|c|c|c|}
\hline \multirow{2}{*}{ Classifier } & \multirow{k}{*}{} & Selftraining \\
\cline { 2 - 3 } & & SKNN \\
\hline \hline \multirow{3}{*}{ Co2KNN } & 1 & não \\
\cline { 2 - 3 } & 3 & $\operatorname{sim}$ \\
\cline { 2 - 3 } & 5 & $\operatorname{sim}$ \\
\cline { 2 - 3 } & 15 & $\operatorname{sim}$ \\
\hline \multirow{4}{*}{ Co2KNN-FA } & 1 & $\operatorname{sim} *$ \\
\cline { 2 - 3 } & 3 & não \\
\cline { 2 - 3 } & 5 & não \\
\cline { 2 - 3 } & 15 & $\operatorname{sim}$ \\
\hline
\end{tabular}

\subsubsection{Comparação entre Co2KNN e Co2KNN-FA}

Analisando todos os resultados mostrados nas seções anteriores, pode-se observar que o classificador Co2KNN teve uma melhor performance do que o classificador Co2KNN-FA em todos os cenários. Para mostrar essa superioridade estatística, foi aplicado o teste de Wilcoxon para cada valor de $k$, sendo esta diferença sempre significativa, como mostrado na Tabela 5.16.

Tabela 5.16: Co2KNN vs Co2KNN-FA com teste estatístico.

\begin{tabular}{|c|c|c|c|c|c|}
\hline \multirow{2}{*}{ Classifier } & \multirow{2}{*}{$\mathbf{k}$} & \multicolumn{5}{|c|}{ Co2KNN-FA } \\
\cline { 2 - 6 } & & 1 & 3 & 5 & 15 \\
\hline \hline \multirow{3}{*}{ Co2KNN } & 1 & sim & - & - & - \\
\cline { 2 - 6 } & 3 & - & sim & - & - \\
\cline { 2 - 6 } & 5 & - & - & sim & - \\
\cline { 2 - 6 } & 15 & - & - & - & sim \\
\hline
\end{tabular}

Com esses resultados, pode-se argumentar que, embora alguma estratégia recupere um número menor de vizinhos e gere confianças altas de classificação, o Cotraining beneficiase destes casos para ajudar o processo de aprendizado conjunto. Como a estratégia KNN Local sempre recupera $k$ vizinhos, acredita-se que a variabilidade do tamanho do conjunto de exemplos recuperado pela estratégia KNN Global permite obter o desacordo necessário para o sucesso do algoritmo Co2KNN. Considerando a análise feita em relação à melhora da estratégia KNN Global com valores de $k$ superiores a 1 e que o algoritmo Co2KNN consegue melhorar o desempenho também com valores de $k$ superiores a 1 , temse evidência suficiente para afirmar que o comportamento do KNN Global no processo de geração de conjuntos de vizinhos fornece informação importante para o sucesso do algoritmo Co2KNN. 


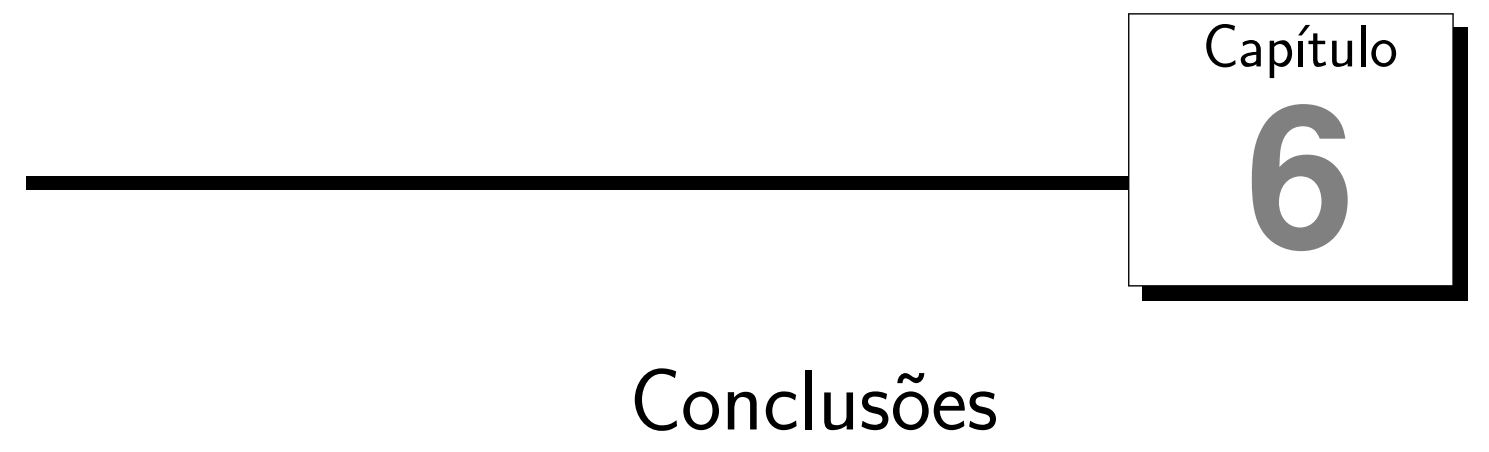

A grande maioria dos cenários reais de classificação apresentam duas características principais: pouca disponibilidade de dados previamente rotulados por especialistas e a existência de uma única visão dos dados. Nesse contexto, faz-se necessário e relevante a geração de algoritmos semi-supervisionados que consigam aproveitar todas as informações disponíveis para gerar modelos robustos em tais circunstâncias, e que, por sua vez, tenham um desempenho comparável com as estratégias supervisionadas.

Neste trabalho foi apresentada a proposta de um novo algoritmo denominado Co2KNN, que é um algoritmo semi-supervisionado que visa gerar um modelo robusto dentro de um cenário com as características já mencionadas. Tal algoritmo combina duas estratégias de recuperação dos vizinhos mais próximos em uma abordagem do estilo Cotraining. As duas estratégias utilizadas foram denominadas KNN Local e KNN Global, sendo que o KNN Global é uma proposta pouco explorada dentro do cenário de classificação. Acredita-se que a alta complexidade computacional do KNN Global quando utilizadas implementações tradicionais desmotivou a pesquisa de tal estratégia. Em tal circunstância, também foi proposta uma estratégia para otimizar o cálculo dos vizinhos mais próximos com a estratégia KNN Global que permite a sua utilização em problemas dentro do contexto de aprendizado semi-supervisionado.

Embora o algoritmo Cotraining seja um algoritmo que assume a existência de mais de uma visão nos dados, ele pode ser adaptado para o cenário mono-visão utilizando a base teórica fornecida pelo Aprendizado Baseado em Desacordo. Por tal, dentro do Capítulo 4 detalhou-se a proposta e foi analisado o desacordo que existe entre os classificadores sob a perspectiva das Redes KNN. Como resultado, mostrou-se que as estratégias KNN Local e KNN Global geram conjuntos com uma variedade suficiente para o sucesso do algoritmo Cotraining especialmente nos casos em que os dados a ser rotulados estão próximos do limite de decisão entre as classes. Nossa proposta se alinha, portanto, ao paradigma do Aprendizado Baseado em Desacordo e reforça a idéia da relevância de "bias" distintos 
entre as duas abordagens, de linguagem (múltiplas representações dos exemplos) ou de algoritmo (múltiplos classificadores) para o sucesso do uso conjunto das mesmas.

Em relação à proposta, cabe ressaltar que o objetivo principal foi desenvolver uma técnica geral para classificação, não estando restrita a algum tipo de domínio, mas possibilitando configurar previamente alguns parâmetros para um comportamento mais adequado ao problema de classificação a ser tratado. Por exemplo, tanto a distância utilizada, o valor do parâmetro $k$, a quantidade de iterações do algoritmo e a quantidade de exemplos rotulados em cada iteração são configuráveis e permitem um maior controle do Co2KNN.

Em relação à avaliação do desempenho da proposta, o Co2KNN foi comparado com outros algoritmos utilizados no estada da arte de classificação, como o Naive Bayes, as Árvores de Decisão e as Máquinas de Vetores de Suporte. O desempenho do Co2KNN foi significativamente melhor do que estes algoritmos, sugerindo que a combinação de classificadores que combinem informações diferentes é uma estratégia mais indicada para cenários nos quais existem poucos dados rotulados. Adicionalmente, os resultados apresentados no capítulo anterior mostraram que a combinação das estratégias KNN Local e KNN Global é melhor do que a utilização de cada estratégia separadamente, sendo o caso que, mesmo quando o KNN Global gera um classificador fraco, consegue ser a chave para o sucesso do algoritmo Co2KNN. Finalmente, comparou-se o classificador Co2KNN com a estratégia usada no classificador SKNN, sendo que o SKNN tem uma idéia similar com o Co2KNN, porém, ao invés de gerar um classificador com cada conjunto de vizinhos recuperados, o SKNN gera um conjunto ampliado combinando os elementos de cada conjunto. O classificador Co2KNN mostrou-se com um melhor desempenho em todos os casos, exceto quando $k=1$. A análise dos conjuntos de vizinhos recuperados mostrou que o motivo principal do desempenho inferior do Co2KNN, quando $k=1$, é devido ao fato da estratégia KNN Global recuperar conjuntos vazios em uma parte considerável dos casos quando $k=1$. Por tal motivo, recomenda-se configurar o parâmetro $k$ com valores maiores do que 1 e menores do que o número inicial de dados rotulados $\left|X_{L}\right|$, a fim de evitar a degradação do algoritmo. Além disso, os experimentos realizados mostraram que os conjuntos de vizinhos recuperados pelo KNN Global são geralmente menores do que o KNN Local, gerando confianças diferentes no processo de classificação.

Duas estratégias diferentes para inferir o rótulo a partir dos conjuntos de dados recuperados foram testadas. A primeira é baseada na votação majoritária, sendo o classificador resultante chamado de Co2KNN. A segunda modifica as confianças geradas pela votação majoritária multiplicando-as por um fator de ajuste que é definido como a relação entre arestas da estratégia respectiva dividia pela quantidade total de arestas (tanto de entrada quanto de saída) conectadas ao novo exemplo. Esta estratégia foi chamada de classificador Co2KNN-FA. O algoritmo Co2KNN, entretanto, mostrou um melhor desempenho em todos os cenários. Acredita-se que o fator de ajuste sempre desfavorece o classificador baseado na estratégia KNN Global, devido a esta gerar conjuntos menores e, portanto, a sua confiança é negativamente influenciada pelo fator de correção. Nesse sentido, as 
confianças calculadas pelos classificadores KNN Global, embora possam ser altas quando existem poucas arestas, são importantes dentro do processo de treino do Co2KNN.

Em relação à usabilidade do algoritmo Co2KNN, mostrou-se independente do domínio, pois esta necessita somente de uma matriz de similaridade para o processo de treino e predição de rótulos. Entretanto, devido ao fato a que a complexidade final do algoritmo Co2KNN é $O\left(\operatorname{loops}\left(l^{2}+u * l\right)\right)$, e $l$ cresce proporcionalmente ao valor de loops. Por tal motivo, recomenda-se que o parâmetro loops não tenha um valor muito alto, pois a complexidade pode subir para $O\left(\operatorname{loops}\left(u^{2}\right)\right)$, sendo este um valor restritivo para problemas com muitos dados. Além disso, o algoritmo Co2KNN foi proposto especificamente para trabalhar com domínios mono-visão, porém, ressalta-se que não está restrito a este domínio, pois poderia utilizar-se dois classificadores KNN Local e Global a mais para cada visão disponível, gerando uma abordagem de comitê de classificadores do estilo Cotraining. Finalmente, nos experimentos foram utilizadas bases com duas classes, para uma melhor avaliação dos resultados, porém, o algoritmo Co2KNN não está restrito a este cenário. Para bases com mais classes, as modificações gerais do algoritmo Co2KNN seriam definidas na quantidade de exemplos a serem rotulados em cada iteração. Em outras palavras, ao invés de ser $n+p$ exemplos rotulados em cada iteração, seriam $c_{1}+c_{2}+c_{3}+\ldots+c_{n}$ os exemplos rotulados, sendo $c_{i}$ a quantidade de exemplos da clase $i$ a ser rotulados em cada iteração. Note-se que a relação entre os diferentes $c_{i}$ deveria ser uma estimação da relação real dos dados para permitir um melhor processo de treinamento.

\subsection{Principais Contribuições}

As principais contribuições deste trabalho estão relacionadas a seguir.

1. Desenvolvimento de uma técnica de aprendizado semi-supervisionado independente do domínio.

2. Desenvolvimento de uma estratégia de otimização para o algoritmo KNN Global.

3. Desenvolvimento de dois classificadores baseados na técnica de aprendizado semisupervisionado.

4. Disponibilização da implementação das diferentes estratégias e técnicas utilizadas.

As técnicas comentadas deram origem às seguintes publicações:

1. LAGUNA, G. V., Lopes, A. A. (2009). A Mult-view Approach for Semi-Supervised Scientific Paper Classification. In: XXIV SBBD- V Workshop em Algoritmos e Aplicações de Mineração de dados, 2009, Fortaleza - Brazil. Proc. V WAAMD, 2009. p. 26-33.

2. LAGUNA, G. V., Lopes, A. A. (2010). Combining Local and Global KNN With Cotraining. In: 19th European Conference on Artificial Intelligence - ECAI 2010, Lisboa, Portugal. 2010. 


\subsection{Trabalhos Futuros}

Como trabalhos futuros, pretende-se estender a avaliação do Co2KNN, comparando-o com outras abordagens semi-supervisionadas, como as SVM semi-supervisionadas e os métodos baseados em grafos. Especificamente quanto aos métodos baseados em grafos, pretende-se estudá-los com maior detalhe, pois encontram-se bastante relacionados com a proposta feita neste trabalho. Nesse contexto, o algoritmo de propagação de rótulo mostrase como uma alternativa interessante a ser estudada na classificação semi-supervisionada baseada em grafos.

Dentro do aprendizado de máquina, o aprendizado ativo tem ganho um interesse muito grande por parte da comunidade científica nos últimos anos, pois é um paradigma de aprendizado que aproveita o conhecimento dos especialistas. Nesse sentido, pretende-se estudar a integração do aprendizado ativo dentro do processo de treinamento do algoritmo Co2KNN. Assim, o algoritmo Co2KNN mostra-se interessante para a detecção de exemplos nos quais as estratégias KNN Local e KNN Global discordam com alta confiança (esses pontos são chamados de Pontos de Desacordo), sendo estes pontos possíveis candidatos a serem rotulados manualmente pelo especialista. Esta abordagem já está sendo desenvolvida em parceria do grupo de pesquisa com o investigador da Universidade de Porto, Prof. Alípio Jorge.

Além disso, pretende-se estudar a formação dos grafos de similaridade para o desempenho do Co2KNN. Para tal motivo, pretende-se utilizar o arcabouço teórico fornecido pelas Redes Complexas no processo de caracterização e análise de tais grafos. As diferentes medidas usadas para descrever as Redes Complexas podem ser utilizadas para criar alguma heurística que permita recuperar os vizinhos mais próximos com a estratégia KNN Global de uma forma mais eficiente.

Por fim, pretende-se analisar e estudar a aplicação do algoritmo Co2KNN em domínios com mais de duas classes. 


\section{Referências Bibliográficas}

Abney, S. Bootstrapping. In: Proceedings of the 40th Annual Meeting of the Association for Computational Linguistics, Morristown, NJ, USA: Association for Computational Linguistics, 2002, p. 360-367.

Abu-Mostafa, Y. S. Machines that learn from hints. Scientific American, v. 272, n. 4, p. 64-69, 1995.

Agrawal, R.; Imieliński, T.; Swami, A. Mining association rules between sets of items in large databases. In: SIGMOD '93: Proceedings of the 1993 ACM SIGMOD international conference on Management of data, New York, USA: ACM, 1993, p. 207216.

Aha, D. W.; Kibler, D.; Albert, M. K. Instance-based learning algorithms. $M a-$ chine Learning, v. 6, n. 1, p. 37-66, 1991.

Anderson, T. W. Asymptotic theory for principal components analysis. Annals of Mathematical Statistics, v. 34, n. 1, p. 123-148, 1963.

Balcan, M.; Blum, A.; YAng, K. Co-training and expansion: Towards bridging theory and practice. In: SAul, L.; Weiss, Y.; Bottou, L., eds. in Neural Information Processing Systems, MIT Press, Cambridge, MA, 2005, p. 89-96.

Barlow, H. B. Unsupervised learning. In: Hinton, G. E.; Sejnowski, T. J., eds. Unsupervised learning: foundations of neural computation, 1 ed, Capítulo 1, MIT Press. Cambridge, MA, p. 1-17, 1999.

Belkin, M.; Niyogi, P.; Sindhwani, V. Manifold regularization: A geometric framework for learning from labeled and unlabeled examples. Journal of Machine Learning Research, v. 7, p. 2399-2434, 2006.

Bennett, K. P.; Demiriz, A. Semi-supervised support vector machines. v. 11, p. $368-374,1998$.

Bentley, J. L. Multidimensional binary search trees used for associative searching. Communications of the ACM, v. 18, n. 9, p. 509-517, 1975. 
Berger, J. O. Statistical decision theory and bayesian analysis. Springer Series in Statistics (Second ed.), 1985.

Bishop, C. M. Neural networks for pattern recognition. Oxford, England: Oxford University Press, 1995.

Blum, A.; Chawla, S. Learning from labeled and unlabeled data using graph mincuts. In: Proceedings of the Eighteenth International Conference on Machine Learning, Massachussets, USA: Morgan Kaufmann Publishers Inc., 2001, p. 19-26.

Blum, A.; Lafferty, J.; Rwebangira, M. R.; Reddy, R. Semi-supervised learning using randomized mincuts. In: Proceedings of the 21st International Conference on Machine Learning, Alberta, Canada: ACM, 2004, p. 97-104.

Blum, A.; Mitchell, T. Combining labeled and unlabeled data with co-training. In: COLT' 98: Proceedings of the eleventh annual conference on Computational learning theory, New York, USA: ACM, 1998, p. 92-100.

Bodo, Z.; Minier, Z. On supervised and semi-supervised k-nearest neighbor algorithms. Studia Universitatis Babes-Bolyai, v. 53, n. 2, p. 79-92, 2008.

Boriah, S.; Chandola, V.; Kumar, V. Similarity measures for categorical data: A comparative evaluation. In: Siam International Conference on Data Mining, Georgia, USA: SIAM, 2008, p. 243-254.

Brito, M. R.; Chavez, E. L.; Quiroz, A. J.; Yukich, J. E. Connectivity of the mutual k-nearest-neighbor graph in clustering and outlier detection. Statistics and Probability Letters, v. 35, n. 1, p. 33-42, 1997.

Chakrabarti, S.; Dom, B.; Indyk, P. Enhanced hypertext categorization using hyperlinks. In: SIGMOD '98: Proceedings of the 1998 ACM SIGMOD international conference on Management of data, New York, NY, USA: ACM, 1998, p. 307-318.

Chang, C.-L. Finding prototypes for nearest neighbor classifiers. IEEE Transactions on Computers, v. 23, n. 11, p. 1179-1184, 1974.

Chapelle, O.; Scholkopf, B.; Zien, A. Semi-supervised learning. MIT Press, Cambridge, MA, 2006.

Chapelle, O.; Weston, J.; Scholkopf, B. Cluster kernels for semi-supervised learning. In: Saul, L.; Weiss, Y.; Bottou, L., eds. Advances in Neural Information Processing Systems, MIT Press, Cambridge, MA, 2003, p. 585-592.

Collins, M.; Singer, Y. Unsupervised models for named entity classification. In: Conference on empirical methods in natural language processing and very large corpora, University of Maryland, USA, 1999, p. 100-110. 
Comon, P. Independent component analysis, a new concept? Signal Process., v. 36, n. 3, p. 287-314, 1994.

Cong, G.; Lee, W. S.; Wu, H.; LiU, B. Semi-supervised text classification using partitioned em. In: 11 th Int. Conference on Database Systems for Advanced Applications (DASFAA), Kyoto, Japan, 2004, p. 482-493.

Cover, T.; HART, P. Nearest neighbor pattern classification. Information Theory, IEEE Transactions on, v. 13, n. 1, p. 21-27, 1967.

Cozman, F.; Cohen, I. Risks of semi-supervised learning: How unlabeled data can degrade performance of generative classifiers. In: ChApelle, O.; SCHÃ $₫$ LKOPF, B.; Zien, A., eds. Semi-Supervised Learning, 1 ed, Capítulo 4, Springer-Verlag, p. 57-72, 2006.

Dasgupta, S.; Littman, M. L.; McAllester, D. A. Pac generalization bounds for co-training. Advances in Neural Information Processing Systems, v. 14, p. 375-382, 2002 .

DeCAESTECKeR, C. Finding prototypes for nearest neighbour classification by means of gradient descent and deterministic annealing. Pattern Recognition, v. 30, n. 2, p. 281 $-288,1997$.

Dellaert, F. The expectation maximization algorithm. Relatório Técnico GIT-GVU02-20, College of Computing, Georgia Institute of Technol, 2002.

Dempster, A. P.; Laird, N. M.; Rubin, D. B. Maximum likelihood from incomplete data via the EM algorithm. Journal of the Royal Statistical Society. Series B (Methodological), v. 39, n. 1, p. 1-38, 1977.

DEMŠAR, J. Statistical comparisons of classifiers over multiple data sets. J. Mach. Learn. Res., v. 7, p. 1-30, 2006.

Domingos, P. Rule induction and instance-based learning a unified approach. In: IJCAI'95: Proceedings of the 14th international joint conference on Artificial intelligence, San Francisco, CA, USA: Morgan Kaufmann Publishers Inc., 1995, p. 1226-1232.

Druck, G.; Pal, C.; McCallum, A.; Zhu, X. Semi-supervised classification with hybrid generative/discriminative methods. In: KDD '0\%: Proceedings of the 13th ACM SIGKDD international conference on Knowledge discovery and data mining, San Jose, California, USA: ACM, 2007, p. 280-289.

DUDANI, S. A. The distance-weighted k-nearest-neighbor rule. IEEE Transactions on Systems, Man, and Cybernetics, v. 6, n. 4, p. 325-327, 1976.

Eppstein, D.; Paterson, M.; Yao, F. F. On nearest-neighbor graphs. Discrete and Computational Geometry, p. 263-282, 1997. 
Fayed, H. A.; Atiya, A. F. A novel template reduction approach for the k-nearest neighbor method. Trans. Neur. Netw., v. 20, n. 5, p. 890-896, 2009.

Feldman, R.; Sanger, J. The text mining handbook: Advanced approaches in analyzing unstructured data. New York, USA: Cambridge University Press, 2006.

FRALICK, S. Learning to recognize patterns without a teacher. Information Theory, IEEE Transactions on, 1967.

Furnkranz, J. Separate-and-conquer rule learning. Artificial Intelligence Review, v. 13, p. 3-54, 1999.

Gantz, J. F.; Chute, C.; Minton, S.; Reinsel, D.; Schlichting, W.; Toncheva, A. The diverse and exploiding digital universe. External Publication of IDC sponsored by EMC, p. 1-16, 2008.

GATEs, G. The reduced nearest neighbor rule. IEEE Transactions on Information Theory, v. 18, n. 3, p. 431-433, 1972.

Geman, S.; Geman, D. Stochastic relaxation, gibbs distributions, and the bayesian restoration of images. Readings in uncertain reasoning, p. 452-472, 1990.

Getoor, L.; Diehl, C. P. Link mining: a survey. SIGKDD Explor. Newsl., v. 7, n. 2 , p. $3-12,2005$.

Getoor, L.; TASKAR, B. Introduction to statistical relational learning (adaptive computation and machine learning). The MIT Press, 2007.

GiL-PitA, R.; YAO, X. Using a genetic algorithm for editing k-nearest neighbor classifiers. In: Intelligent Data Engineering and Automated Learning - IDEAL 200\%, Heidelberg, DE: Springer Berlin, 2007, p. 1141-1150.

Goldman, S. A.; Zhou, Y. Enhancing supervised learning with unlabeled data. In: Proceedings of the Seventeenth International Conference on Machine Learning, San Francisco, CA, USA: Morgan Kaufmann Publishers Inc., 2000, p. 327-334.

Goto, M.; Ishida, T.; Hirasawa, S. Statistical evaluation of measure and distance on document classification problems in text mining. In: CIT 'O\%: Proceedings of the 7th IEEE International Conference on Computer and Information Technology, Washington, DC, USA: IEEE Computer Society, 2007, p. 674-673.

Gowda, K.; Krishna, G. Agglomerative clustering using the concept of mutual nearest neighborhood. Pattern Recognition, v. 10, n. 2, p. 105-112, 1978.

Guan, D.; Yuan, W.; LeE, Y.-K.; LeE, S. Semi-supervised nearest neighbor editing. In: IEEE International Joint Conference on Neural Networks, 2008. IJCNN 
2008. (IEEE World Congress on Computational Intelligence), Hong Kong: IEEE International, 2008, p. 1183-1187.

HAN, E.-H. Text categorization using weight adjusted k-nearest neighbor classification. Tese de Doutorado, University of Minesota, USA, 1999.

Harwood, D.; Subbarao, M.; Hakalahti, H.; Davis, L. S. A new class of edge-preserving smoothing filters. Pattern Recogn. Lett., v. 6, n. 3, p. 155-162, 1987.

Hastie, T.; Tibshirani, R.; Friedman, J. H. The elements of statistical learning. Corrected ed. Springer, 2003.

Hein, M.; Luxburg, U. V. Introduction to graph-based semi-supervised learning. Machine Learning Summer School 2007. Practical Session on Graph-based Algorithms in Machine Learning. Tubingen, Germany., 2007.

HuAng, Z. A fast clustering algorithm to cluster very large categorical data sets in data mining. In: II Workshop on Research Issues on Data Mining and Knowledge Discovery - DMKD, Tucson, Arizona, USA: ACM SIGMOD, 1997, p. 1-8.

Hutter, M.; Servedio, R. A.; TAkimoto, E. Cluster identification in nearestneighbor graphs. In: Proceedings of Algorithmic Learning Theory, 18th International Conference, ALT 200\%, Sendai, Japan: Springer Berlin, 2007, p. 196-210 (Lecture Notes in Computer Science, v.4754).

JACCARD, P. Etude comparative de la distribution florale dans une portion des alpes et des jura. Bulletin de la Société Vaudoise des Sciences Naturelles, v. 37, p. 547-579, 1901.

JiAnG, L.; CAI, Z.; WANG, D.; JiAng, S. Survey of improving k-nearest-neighbor for classification. In: FSKD '0\%: Proceedings of the Fourth International Conference on Fuzzy Systems and Knowledge Discovery, Washington, DC, USA: IEEE Computer Society, 2007, p. 679-683.

JIANG, Y.; ZHOU, Z. Editing training data for knn classifiers with neural network ensemble. In: Lecture Notes in Computer Science, Springer, 2004, p. 356-361.

JoAchims, T. Text categorization with support vector machines: learning with many relevant features. In: NÉDEllec, C.; Rouveirol, C., eds. Proceedings of ECML98, 10th European Conference on Machine Learning, Chemnitz, DE: Springer Verlag, Heidelberg, DE, 1998, p. 137-142.

JoAchims, T. Transductive inference for text classification using support vector machines. ICML, 1999.

Karypis, G.; Han, E.-H.; Kumar, V. Chameleon: hierarchical clustering using dynamic modeling. Computer, v. 32, n. 8, p. 68-75, 1999. 
Kaufman, L.; Rousseeuw, P. J. Finding groups in data: An introduction to cluster analysis. Wiley-Interscience, 1990.

Kohonen, T.; Schroeder, M. R.; Huang, T. S., eds. Self-organizing maps. Secaucus, NJ, USA: Springer-Verlag New York, Inc., 2001.

Kuncheva, L. I. Combining pattern classifiers: Methods and algorithms. WileyInterscience, 2004.

Kuncheva, L. I.; Whitaker, C. J. Measures of diversity in classifier ensembles and their relationship with the ensemble accuracy. Mach. Learn., v. 51, n. 2, p. 181-207, 2003.

Laguna, V.; Lopes, A. A. A mult-view approach for semi-supervised scientific paper classification. In: XXIV SBBD. V Workshop em Algoritmos e Aplicações de Mineração de dados, Fortaleza, CE, Brazil, 2009, p. 26-33.

Lathia, N.; Hailes, S.; CAPRA, L. knn cf: a temporal social network. In: RecSys '08: Proceedings of the 2008 ACM conference on Recommender systems, New York, NY, USA: ACM, 2008, p. 227-234.

LeE, M. D.; Welsh, M. An empirical evaluation of models of text document similarity. In: XXVII Annual Conference of the Cognitive Science Society (CogSci2005), Erlbaum, 2005, p. 1254-1259.

Leicht, E. A.; Holme, P.; Newman, M. E. J. Vertex similarity in networks. Physical Review E, v. 73, p. 0261201-0261210, 2006.

LI, M. Tri-training: Exploiting unlabeled data using three classifiers. IEEE Trans. on Knowl. and Data Eng., v. 17, n. 11, p. 1529-1541, member-Zhi-Hua Zhou, 2005.

LI, M.; ZHOU, Z.-H. Improve computer-aided diagnosis with machine learning techniques using undiagnosed samples. Systems, Man and Cybernetics, Part A: Systems and Humans, IEEE Transactions on, v. 37, n. 6, p. 1088-1098, 2007.

Li, T.; Ogihara, M. Semisupervised learning from different information sources. Knowledge and Information Systems, v. 7, n. 3, p. 289-309, 2005.

Ling, C. X.; Du, J.; Zhou, Z.-H. When does co-training work in real data? In: PAKDD '09: Proceedings of the 13th Pacific-Asia Conference on Advances in Knowledge Discovery and Data Mining, Berlin, Heidelberg: Springer-Verlag, 2009, p. 596-603.

Liu, H.; Motoda, H. Feature selection for knowledge discovery and data mining. Norwell, MA, USA: Kluwer Academic Publishers, 1998.

LLOYD, S. Least squares quantization in pcm. Information Theory, IEEE Transactions on, v. 28, n. 2, p. 129-137, 1982. 
Lu, Q.; Getoor, L. Link-based classification using labeled and unlabeled data. In: Proceedings of the ICML-2003 Workshop on The Continuum from Labeled to Unlabeled Data, 2003.

Lyman, P.; Varian, H. R.; Charles, P.; Good, N.; Jordan, L. L.; Pal, J. How much information? Relatório Técnico, Regents of the University of California, 2003.

Mahalanobis, P. C. On the generalized distance in statistics. In: Proceedings of the National Institute of Sciences of India, Indian National Science Academy, 1936, p. 49-55.

McCallum, A.; Nigam, K. Employing EM and pool-based active learning for text classification. In: Proceedings of the Fifteenth International Conference on Machine Learning, Wisconsin, USA: Morgan Kaufmann Publishers Inc., 1998, p. 350-358.

Mccallum, A. K.; Mitchell, T. Text classification from labeled and unlabeled documents using em. In: Machine Learning, 2000, p. 103-134.

Mclachlan, G. J.; Krishnan, T. The EM algorithm and extensions. 1 ed. New York, NY, USA: Wiley-Interscience, 1996.

MerRIs, R. Laplacian graph eigenvectors. Linear Algebra and its Applications, v. 278, n. 1-3, p. 221-236, 1998.

Mitchell, T. M.; Blum, A. Machine learning. New York, USA: McGraw-Hill Education (ISE Editions), 1997.

Moreno, P. J.; Agarwal, S. An experimental study of semi-supervised EM algorithms in audio classification and speaker identification. Relatório Técnico, Hewlett-Packard Company - HP Laboratories Cambridge, MA, 2003.

Motta, R. C.; Lopes, A. A. Uso de redes complexas na classificação relacional. Dissertação de Mestrado, ICMC-USP, São Carlos, SP, 2009.

Motta, R. C. D.; Almeida, L.; A. Lopes, A. Rede probabilísticas baseada em similaridade na exploração de comunidades. In: I Workshop on Web and Text Intelligence (WTI 2008), Salvador, Bahia, Brazil, 2008, p. 11-18.

Muslea, I.; Minton, S.; Knoblock, C. A. Active + semi-supervised learning = robust multi-view learning. In: Proceedings of the Nineteenth International Conference on Machine Learning, Sydney, AU: Morgan Kaufmann Publishers Inc., 2002, p. 435442.

Newman, M. E. J. The structure and function of complex networks. SIAM Review, v. 45, p. 167-256, 2003. 
Nigam, K.; Ghani, R. Understanding the behavior of co-training. In: Proceedings of KDD-2000 Workshop on Text Mining, 2000.

Nock, R.; Sebban, M.; Bernard, D. A simple locally adaptive nearest neighbor rule with application to pollution forecasting. International Journal of Pattern Recognition and Artificial Intelligence, v. 17, n. 8, p. 1369-1382, 2003.

Nock, R.; Sebban, M.; Jabby, P. A symmetric nearest neighbor learning rule. In: EWCBR '00: Proceedings of the 5th European Workshop on Advances in Case-Based Reasoning, London, UK: Springer-Verlag, 2000, p. 222-233.

Oкамото, S.; Yugami, N. Effects of domain characteristics on instance-based learning algorithms. Theoretical Computter Science, v. 298, n. 1, p. 207-233, 2003.

Papadimitriou, C. H.; Bentley, J. L. A worst-case analysis of nearest neighbor searching by projection. In: Proceedings of the rth Colloquium on Automata, Languages and Programming, London, UK: Springer-Verlag, 1980, p. 470-482.

Pierce, D.; CARDie, C. Limitations of co-training for natural language learning from large datasets. In: Proceedings of the 2001 Conference on Empirical Methods in Natural Language Processing, 2001, p. 1-9.

Powell, M. J. D. Radial basis functions for multivariable interpolation: a review. Algorithms for approximation, p. 143-167, 1987.

Quinlan, J. Induction of decision trees. Machine Learning, v. 1, n. 1, p. 81-106, 1986.

RAYID, G. Combining labeled and unlabeled data for text classification with a large number of categories. In: ICDM '01: Proceedings of the 2001 IEEE International Conference on Data Mining, Washington, DC, USA: IEEE Computer Society, 2001, p. $597-598$.

Ritter, G.; Woodruff, H.; Lowry, S.; Isenhour, T. An algorithm for a selective nearest neighbor decision rule. IEEE Transactions on Information Theory, v. 21, n. 6, p. 665-669, 1975.

Russell, S. J.; Norvig Artificial intelligence: A modern approach. 3 ed. New Jersey, USA: Prentice Hall, 2003.

SA, V. R. Learning classification with unlabeled data. In: Cowan, J. D.; Tesauro, G.; Alspector, J., eds. Proceedings of the Neural Information Processing Systems, San Francisco, CA: Morgan Kaufmann Publishers, 1993, p. 112-119.

Santini, S.; Jain, R. Similarity measures. IEEE Transactions on Pattern Analysis and Machine Intelligence, v. 21, n. 9, p. 871-883, 1999.

Schaeffer, S. Graph clustering. Computer Science Review, v. 1, n. 1, p. 27-64, 2007. 
ScudDER, H. Probability of error of some adaptive pattern-recognition machines. Information Theory, IEEE Transactions on, p. 363-371, 1965.

Sindhwani, V.; Niyogi, P.; Belkin, M. Beyond the point cloud: from transductive to semi-supervised learning. In: Proceedings of the 22nd international conference on Machine learning, Bonn, Germany: ACM, 2005a, p. 824-831.

Sindhwani, V.; Niyogi, P.; Belkin, M. A co-regularization approach to semisupervised learning with multiple views. In: Workshop on Learning with Multiple Views, Proceedings of International Conference on Machine Learning, Bonn, Germany: ACM, 2005b, p. 74-79.

Singh, A.; Nowak, R.; Zhu, X. Unlabeled data: Now it helps, now it doesn't. In: Koller, D.; Bengio, Y.; Schuurmans, D.; Bottou, L.; Culotta, A., eds. Advances in Neural Information Processing Systems, MIT Press, Cambridge, MA, 2008, p. $1513-1521$.

Song, Y.; Huang, J.; Zhou, D.; Zha, H.; Giles, C. L. Iknn: Informative knearest neighbor pattern classification. In: PKDD 200\%: Proceedings of the 11th European conference on Principles and Practice of Knowledge Discovery in Databases, Berlin, Heidelberg: Springer-Verlag, 2007, p. 248-264.

Specht, D. Probabilistic neural networks. Neural Networks, v. 3, n. 1, p. 109-118, 1990.

Valiant, L. G. A theory of the learnable. Commun. ACM, v. 27, n. 11, p. 1134-1142, 1984.

VAPNIK, V. Estimation of dependences based on empirical data. Secaucus, NJ, USA: Springer Series in Statistics. Springer-Verlag New York, Inc., 1982.

VApnik, V. Transductive inference and semi-supervised learning. In: Chapelle, O.; Scholkopf, B.; Zien, A., eds. Semi-Supervised Learning, 1 ed, Capítulo 24, Springer-Verlag, p. 453-472, 2006.

VAPNIK, V.; STERIN, A. On structural risk minimization or overall risk in a problem of pattern recognition. Automation and Remote Control, v. 10, n. 3, p. 1495-1503, 1977.

VAPNIK, V. N. The nature of statistical learning theory. New York, NY, USA: SpringerVerlag New York, Inc., 1995.

Wang, L.; Khan, L.; Thuraisingham, B. An effective evidence theory based k-nearest neighbor (knn) classification. In: WI-IAT '08: Proceedings of the 2008 IEEE/WIC/ACM International Conference on Web Intelligence and Intelligent Agent Technology, Washington, DC, USA: IEEE Computer Society, 2008, p. 797-801. 
Wang, W.; Zhou, Z.-H. Analyzing co-training style algorithms. In: Proceedings of the 18th European conference on Machine Learning, Berlin, Heidelberg: Springer-Verlag, 2007, p. 454-465.

Watson, I. D. An introduction to case-based reasoning. In: Proceedings of the First United Kingdom Workshop on Progress in Case-Based Reasoning, London, UK: Springer-Verlag, 1995, p. 3-16.

Weber, R.; Schek, H.-J.; Blott, S. A quantitative analysis and performance study for similarity-search methods in high-dimensional spaces. In: VLDB '98: Proceedings of the 24rd International Conference on Very Large Data Bases, San Francisco, CA, USA: Morgan Kaufmann Publishers Inc., 1998, p. 194-205.

Weinberger, K. Q.; SAul, L. K. Distance metric learning for large margin nearest neighbor classification. J. Mach. Learn. Res., v. 10, p. 207-244, 2009.

Weiss, S. M.; Indurkhya, N.; Zhang, T.; Damerau, F. J. Text mining-predictive methods for analyzing unstructured information. New York, USA: Springer Science and Business Media, Inc., ISBN 0-387-95433-3, 2005.

Wilson, D. L. Asymptotic properties of nearest neighbor rule using edited data. IEEE Transactions on Systems, Man and Cybernetics, v. 2, n. 3, p. 408-421, 1972.

Wilson, D. R.; Martinez, T. R. Improved heterogeneous distance functions. Journal of Artificial Intelligence Research, v. 6, p. 1-34, 1997.

Wilson, D. R.; Martinez, T. R. Reduction techniques for instance-based learning algorithms. Machine Learning, v. 38, n. 3, p. 257-286, 2000.

Witten, I. H.; Frank, E. Data mining: Practical machine learning tools and techniques, second edition (morgan kaufmann series in data management systems). San Francisco, CA, USA: Morgan Kaufmann, 2005.

Xiong, H.; Steinbach, M.; Kumar, V. Privacy leakage in multi-relational databases: a semi-supervised learning perspective. The VLDB Journal, v. 15, n. 4, p. 388-402, 2006.

Yan, R.; NAPhade, M. Multi-modal video concept extraction using co-training. Multimedia and Expo, 2005. ICME 2005. IEEE International Conference on, p. 514-517, 2005 .

YAROWSKY, D. Unsupervised word sense disambiguation rivaling supervised methods. In: Meeting of the Association for Computational Linguistics, 1995, p. 189-196.

Zhang, H.; Berg, A.; Maire, M.; Malik, J. Svm-knn: Discriminative nearest neighbor classification for visual category recognition. In: Computer Vision and Pattern Recognition, 2006 IEEE Computer Society Conference on, 2006, p. 2126-2136. 
Zhang, W.; Zheng, Q. Tsfs: A novel algorithm for single view co-training. In: CSO '09: Proceedings of the 2009 International Joint Conference on Computational Sciences and Optimization, Washington, DC, USA: IEEE Computer Society, 2009, p. 492-496.

Zhipeng, X.; Hsu, W.; Zongtian, L.; Lee, M.-L. Snnb: A selective neighborhood based naïve bayes for lazy learning. In: PAKDD '02: Proceedings of the 6th PacificAsia Conference on Advances in Knowledge Discovery and Data Mining, London, UK: Springer-Verlag, 2002, p. 104-114.

Zhou, Y.; Goldman, S. Democratic co-learning. In: ICTAI '04: Proceedings of the 16th IEEE International Conference on Tools with Artificial Intelligence, Washington, USA: IEEE Computer Society, 2004, p. 594-202.

ZHou, Z.-H. When semi-supervised learning meets ensemble learning. In: MCS '09: Proceedings of the 8th International Workshop on Multiple Classifier Systems, Berlin, Heidelberg: Springer-Verlag, 2009, p. 529-538.

Zhou, Z.-H.; LI, M. Semi-supervised regression with co-training. In: IJCAI'05: Proceedings of the 19th international joint conference on Artificial intelligence, San Francisco, CA, USA: Morgan Kaufmann Publishers Inc., 2005, p. 908-913.

ZHou, Z.-H.; LI, M. Semisupervised regression with cotraining-style algorithms. IEEE Trans. on Knowl. and Data Eng., v. 19, n. 11, p. 1479-1493, 2007.

Zhou, Z.-H.; Li, M. Semi-supervised learning by disagreement. Knowledge and Information Systems, in press, p. 1-27, 2009.

Zhou, Z.-H.; Zhan, D.; Yang, Q. Semi-supervised learning with very few labeled training examples. In: Twenty-Second AAAI Conference on Artificial Intelligence (AAAI-07), 2007, p. 675-680.

Zhu, X. Semi-supervised learning with graphs. Tese de Doutorado, School of Computer Science - Carnegie Mellon University, 2005.

Zhu, X. Semi-supervised learning literature survey. Relatório Técnico 1530, Computer Sciences, University of Wisconsin-Madison, 2007.

Zhu, X.; Ghahramani, Z. Learning from labeled and unlabeled data with label propagation. Relatório Técnico CMU-CALD-02-107, Carnagie Mellon University, 2002.

Zhu, X.; Ghahramani, Z.; Lafferty, J. Semi-supervised learning using gaussian fields and harmonic functions. In: Twentieth International Conference on Machine Learning, Washington DC: AAAI Press, 2003, p. 912-919.

Zhu, X.; Goldberg, A. B. Introduction to semi-supervised learning. Morgan \& Claypool Publishers, 2009. 


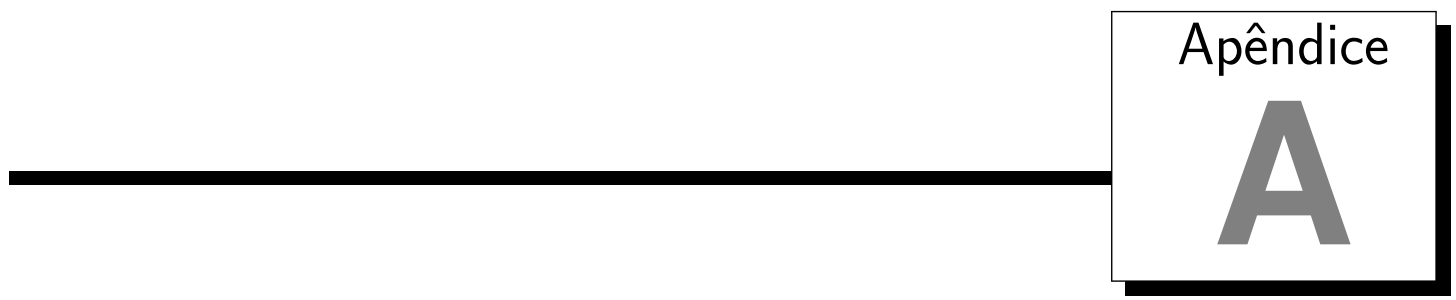

\section{Figuras com o desempenho dos classificadores em relação a K}

Este apêndice contém as Figuras completas dos resultados do desempenho dos classifcadores Co2KNN, KNN Local e KNN Global em relação ao valor de $k$. 


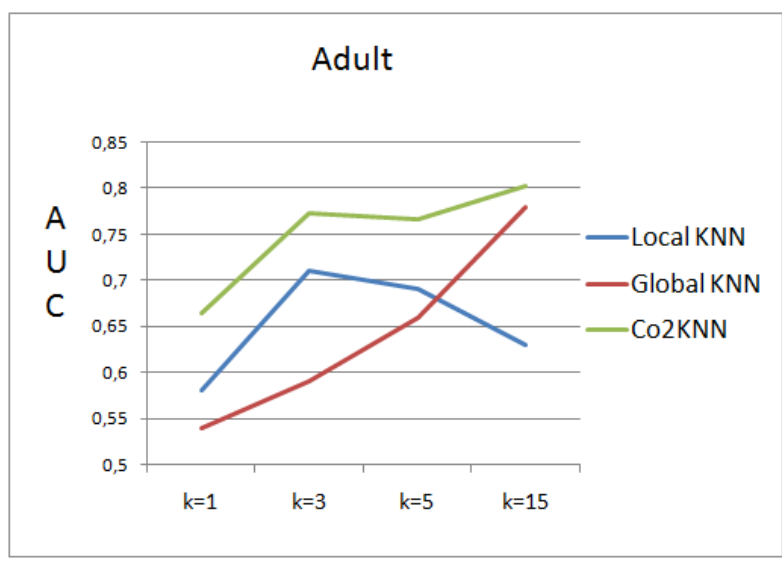

(a)

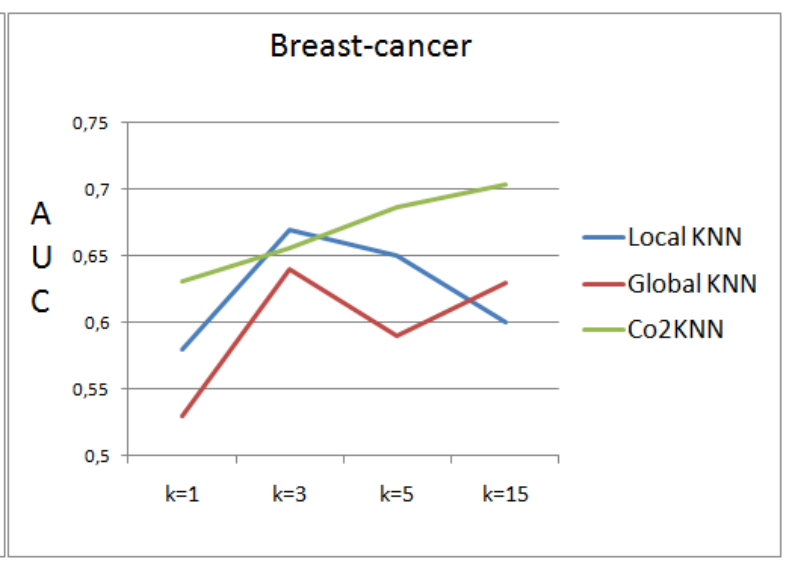

(b)

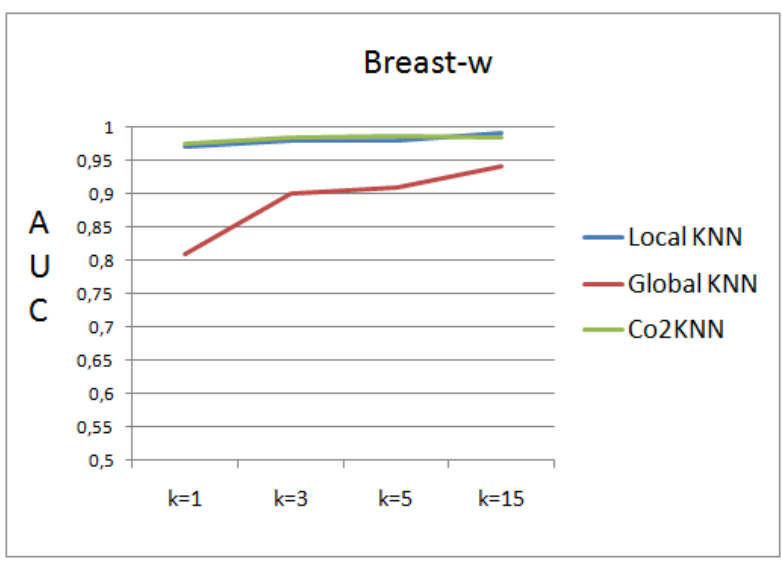

(c)

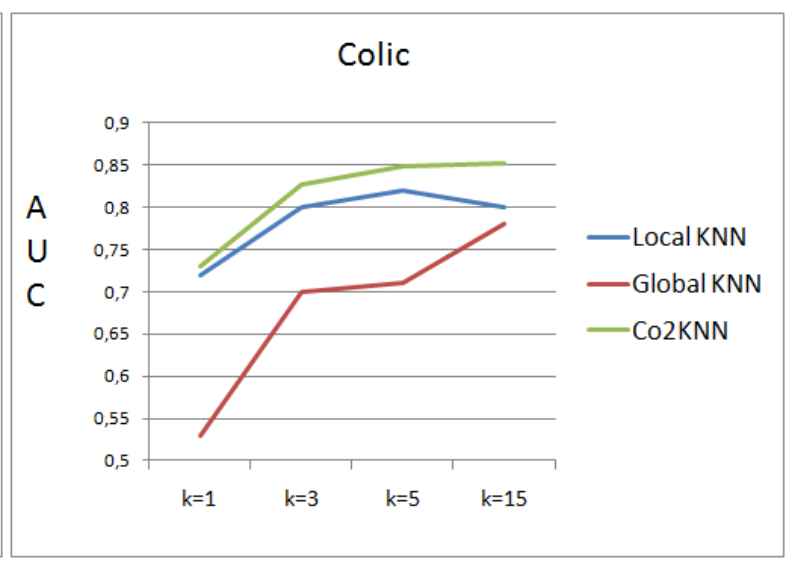

(d)

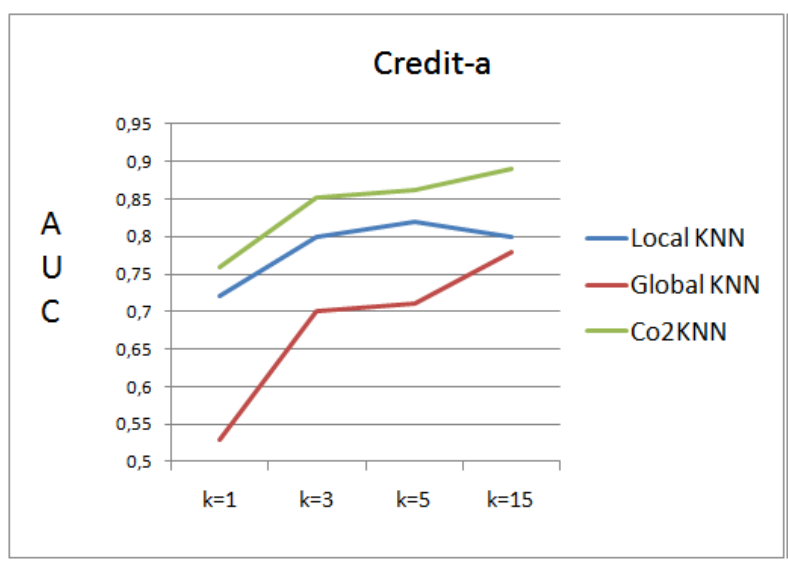

(e)

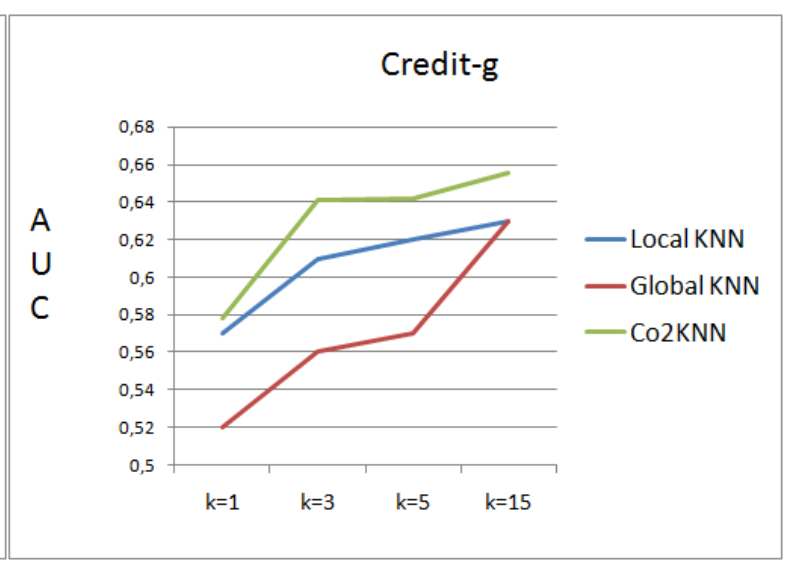

(f)

Figura A.1 


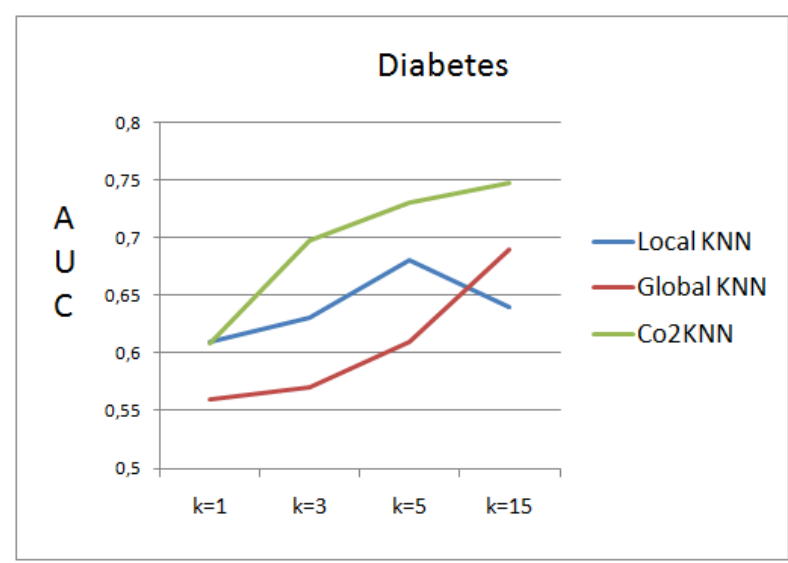

(a)

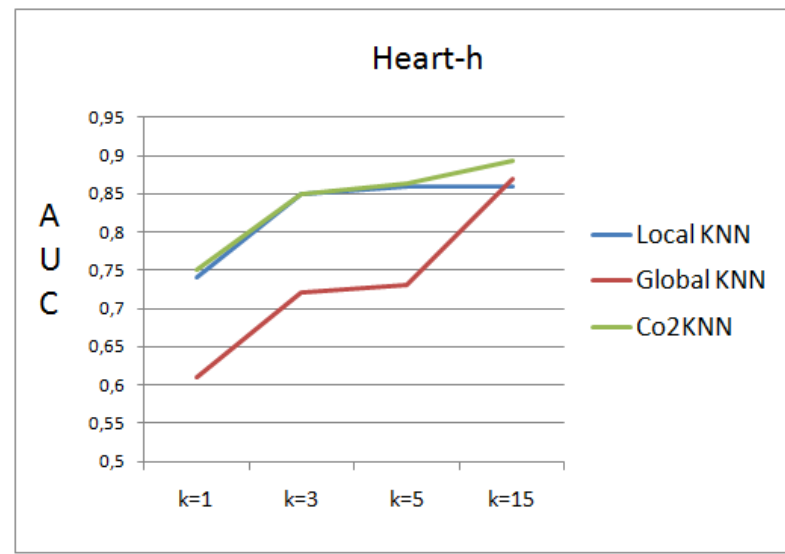

(c)

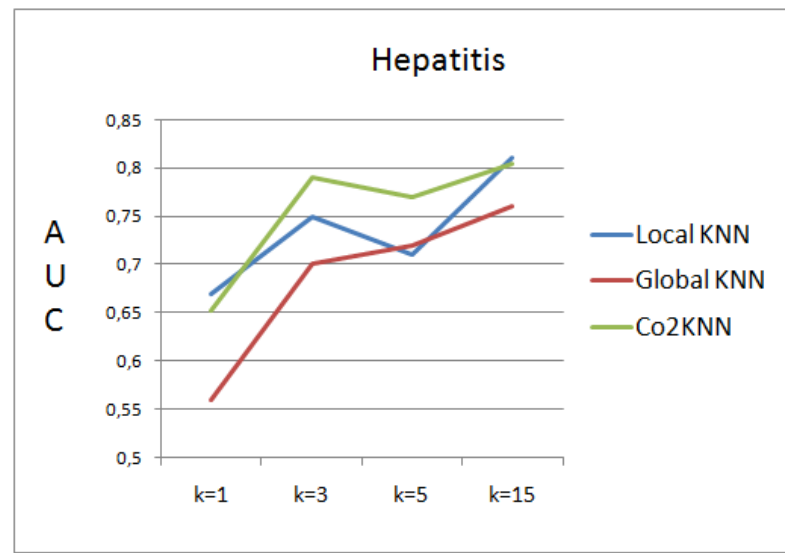

(e)

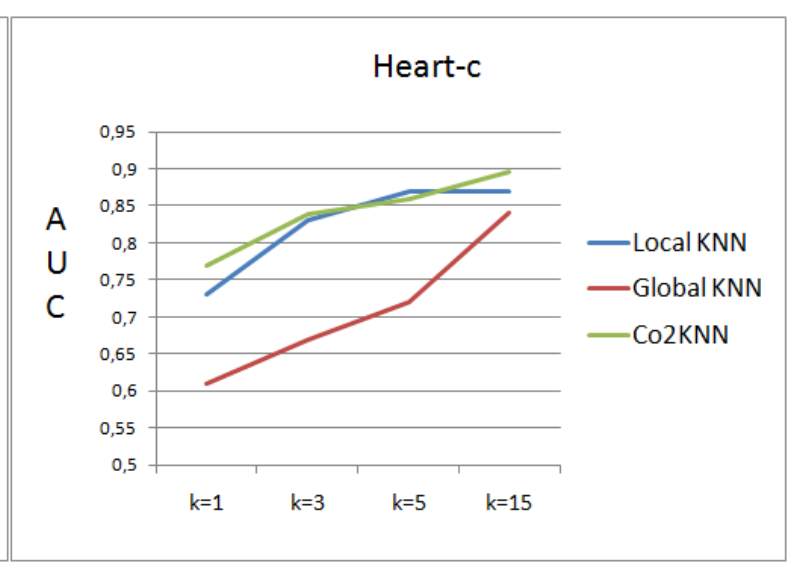

(b)

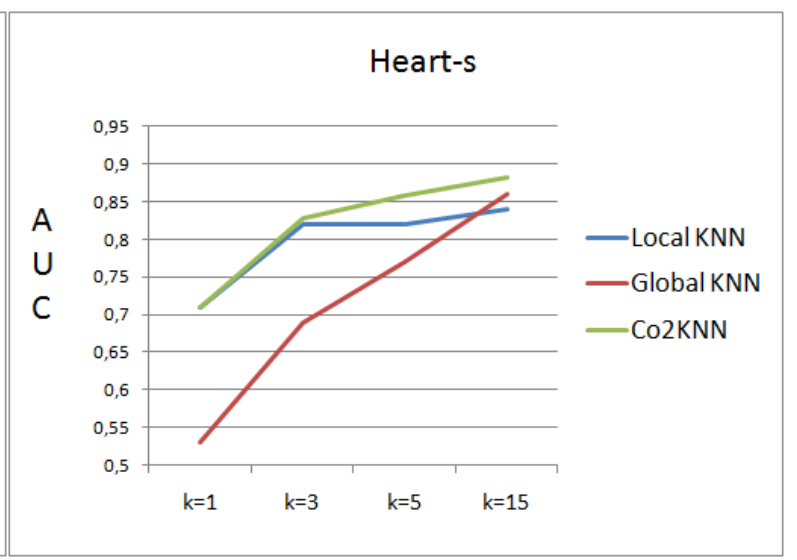

(d)

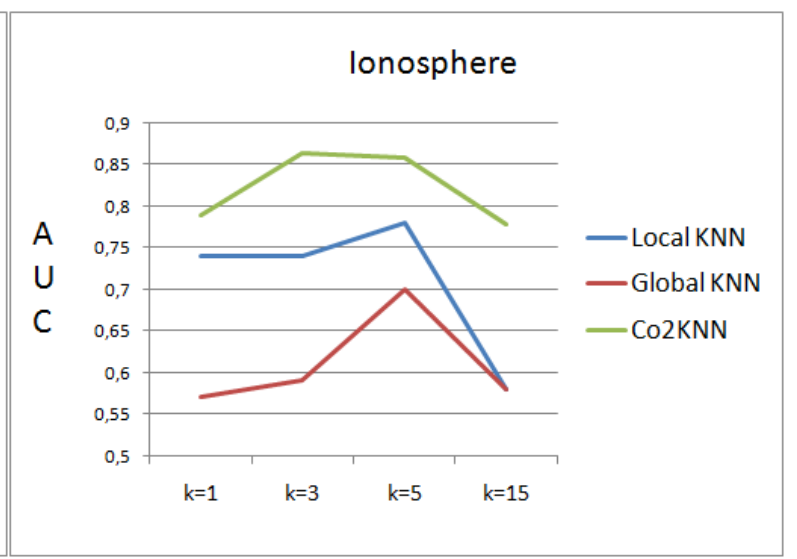

(f)

Figura A.2 


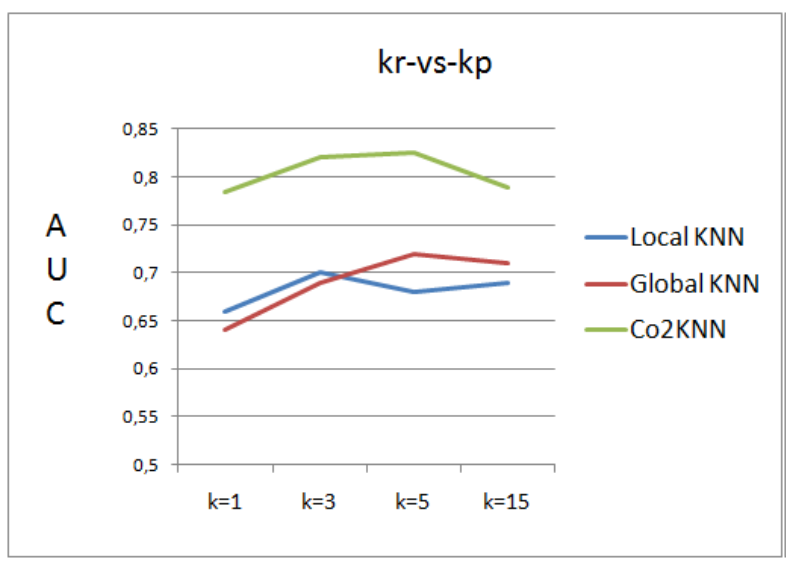

(a)

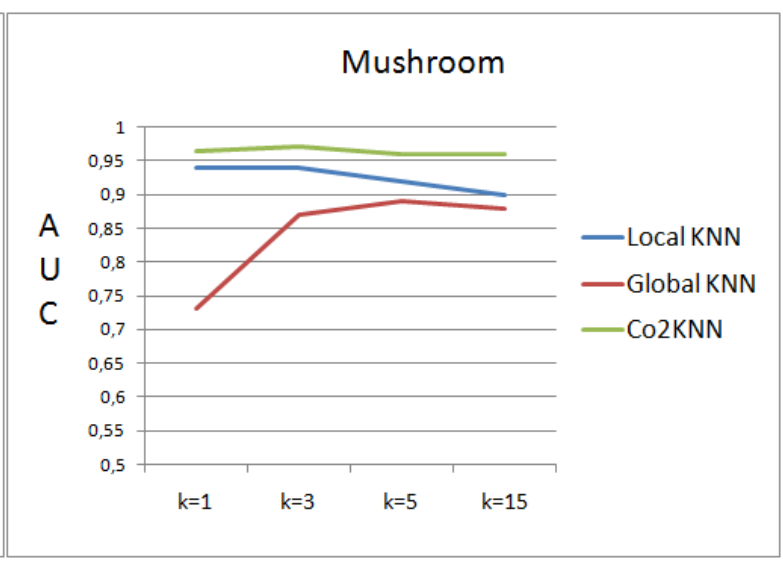

(b)

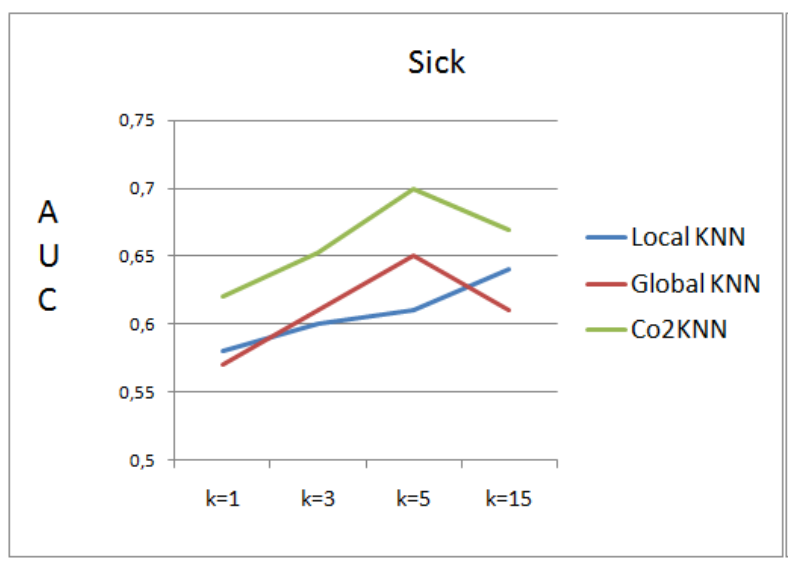

(c)

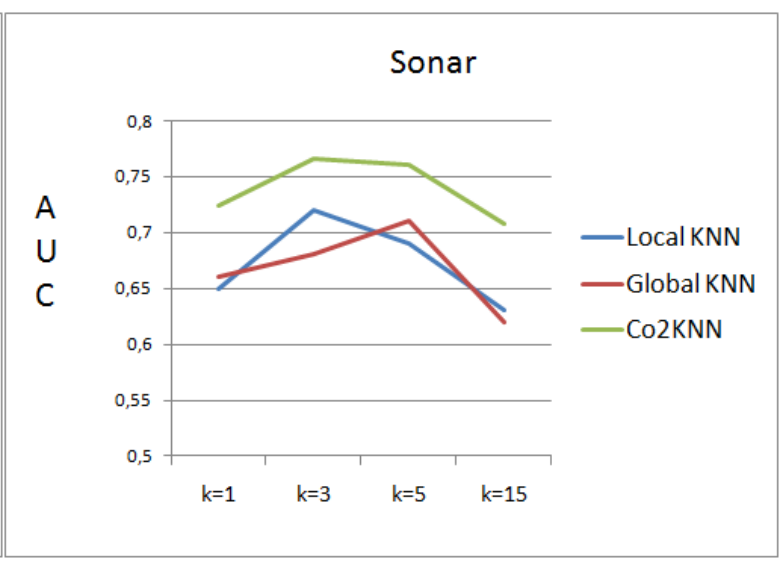

(d)

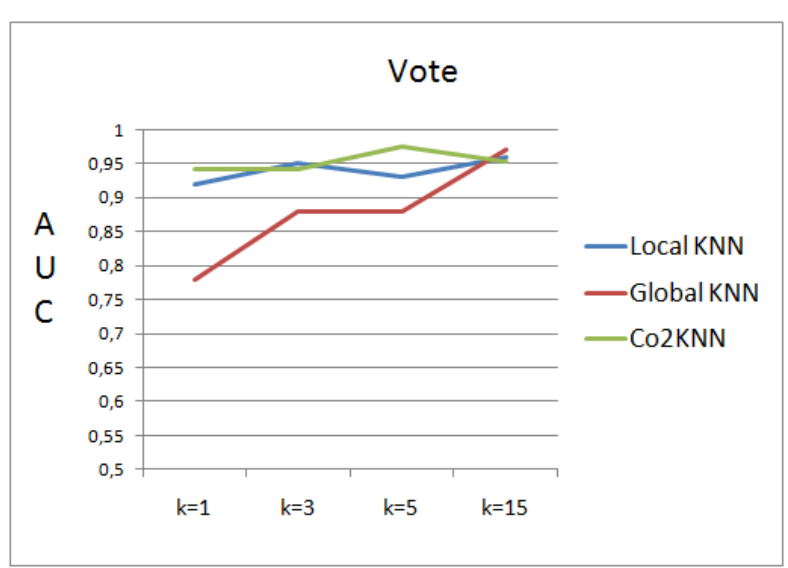

(e)

Figura A.3 